\title{
Simulation study of Hollow Electron Beam Collimation in HL-LHC.*
}

\author{
M. Fitterer ${ }^{\dagger}$ and G. Stancari, A. Valishev \\ Fermi National Accelerator Laboratory, PO Box 500, Batavia, Illinois 60510, USA \\ S. Redaelli \\ CERN, CH-1211 Geneva 23, Switzerland
}

(Dated: Oct. 5, 2016)

\begin{abstract}
Collimation with hollow electron beams is currently one of the most promising concepts for active halo control in the HL-LHC. In this note we evaluate the performance of a hollow electron lens for different HL-LHC scenarios in terms of halo removal rates and the effect of profile imperfections of the electron beam on the proton beam core in case of a pulsed operation of the e-lens.
\end{abstract}

\footnotetext{
* Fermilab is operated by Fermi Research Alliance, LLC under Contract No. DE-AC02-07CH11359 with the United States Department of Energy. This work was partially supported by the US DOE LHC Accelerator Research Program (LARP) and by the European FP7 HiLumi LHC Design Study, Grant Agreement 284404.

$\dagger$ Email: mfittere@fnal.gov
}

This manuscript has been authored by Fermi Research Alliance, LLC under Contract No. DE-AC02-07CH11359 with the U.S. Department of Energy, Office of Science, Office of High Energy Physics. 


\section{CONTENTS}

$\begin{array}{ll}\text { I. Introduction } & 4\end{array}$

$\begin{array}{ll}\text { II. HL-LHC scenarios } & 5\end{array}$

III. HEL position and optics in IR4 6

IV. Halo removal rates for the different HL-LHC scenarios and DC operation $\quad 7$

A. Halo removal rates at flat top for HEL installed at $-40 \mathrm{~m}$ from IP4 and different chromaticity and octupole settings 9

B. Halo removal rates at flat top in the presence of errors and for HEL installed at $-40 \mathrm{~m}$ or $-88.6 \mathrm{~m}$ from IP4 11

C. Halo removal rates during $\beta^{*}$ leveling for HEL installed at $-40 \mathrm{~m}$ from IP4 13

V. Halo removal rates for the different HL-LHC scenarios and pulsed operation 14

VI. Modeling of the halo removal rates $\quad 17$

$\begin{array}{ll}\text { VII. Conclusions } & 19\end{array}$

$\begin{array}{ll}\text { Acknowledgments } & 22\end{array}$

A. DA simulations for luminosity leveling from $0.7 \mathrm{~m}$ to $0.15 \mathrm{~m}$. 24

B. Comparison of fit parameters and average halo removal rates 25

1. Fit parameters and average halo removal rate at flat top 25

a. Fit parameters and average halo removal rate: flat top and different chromaticity and octupole settings for HEL installed at $-40 \mathrm{~m} \quad 25$

b. Fit parameters and average halo removal rate: flat top with magnet errors and HEL at $-40 \mathrm{~m}$ and -88.6 m: $Q^{\prime}=15, I_{\mathrm{MO}}=-550 \mathrm{~A}$

c. Fit parameters and average halo removal rate: flat top with pulsing: $Q^{\prime}=15, I_{\mathrm{MO}}=-550$ A 27

2. Fit parameters: $\beta^{*}$-leveling cases for HEL installed at $-40 \mathrm{~m} \quad 28$

C. FMA analysis $\quad 29$

1. FMA analysis for flat top $\quad 29$

a. FMA analysis: flat top and different chromaticity and octupole settings for HEL installed at $-40 \mathrm{~m} \quad 29$

b. FMA analysis: flat top with magnet errors and HEL at $-40 \mathrm{~m}$ and $-88.6 \mathrm{~m}$ : $Q^{\prime}=15, I_{\mathrm{MO}}=-550 \mathrm{~A}$

c. FMA analysis: flat top with pulsing and $Q^{\prime}=15, I_{\mathrm{MO}}=-550 \mathrm{~A}$ 
2. FMA analysis: $\beta^{*}$-leveling cases for HEL installed at $-40 \mathrm{~m}$

$\begin{array}{ll}\text { D. Losses versus amplitude } & 49\end{array}$

E. Losses versus amplitude at flat top $\quad 49$

a. Losses vs amplitude: flat top and different chromaticity and octupole settings for HEL installed at $-40 \mathrm{~m}$

b. Losses vs amplitude: flat top with magnet errors and HEL at $-40 \mathrm{~m}$ and $-88.6 \mathrm{~m}$ : $Q^{\prime}=15, I_{\mathrm{MO}}=-550 \mathrm{~A} \quad 51$

c. Losses vs amplitude: flat top with pulsing and $Q^{\prime}=15, I_{\mathrm{MO}}=-550 \mathrm{~A}$

1. Losses vs amplitude: : $\beta^{*}$-leveling cases for HEL installed at $-40 \mathrm{~m}$

$\begin{array}{ll}\text { References } & 58\end{array}$ 


\section{INTRODUCTION}

For high energy and high intensity hadron colliders like the HL-LHC, halo control becomes more and more relevant if not necessary for a save machine operation and control of the targeted stored beam energy in the range of several hundred MJ. Past experiments at the Fermilab Tevatron proton-antiproton collider [1] demonstrated a successful halo control with hollow electron beams. In view of an application to the HL-LHC first numerical simulations for the nominal LHC [2-4] have been conducted. In summary, the simulations show a sufficiently high halo removal rate if the beams are colliding, but only very low halo removal rates if the beams are separated. Therefore, in order to clean the tails efficiently and in a short time-span also in case of separated beams, e.g. before the squeeze, the halo removal rate can be increased by pulsing the e-lens $[4,5]$, where two different pulsing patterns are considered:

- random: the e-beam current is modulated randomly,

- resonant: the e-lens is switched on only every $n$th turn with $n=2,3,4, \ldots$.

One of the main reservations about pulsing the e-lens is the possibility of emittance growth due to noise induced on the beam core by the e-lens.

These first numerical simulations were however conducted for the nominal LHC lattice without errors, zero octupole current and a low chromaticity of 3 . As will be shown later in this note, the halo removal rates depend strongly on the non-linearities present in the machine, thus explicitly:

- current of Landau damping octupoles

- magnetic errors

- chromaticity

- non-linearities introduced by the beam-beam interaction

In particular a strong effect of the octupole and chromaticity settings on the halo removal rates in the case of separated beams has been observed which can be explained by the increased tune spread due the octupoles and the continuous swiping of the particles over the excited resonances due to the synchrotron motion. In summary, it is therefore important to simulate more realistic scenarios in order to evaluate the performance of an hollow electron lens for HL-LHC in terms of halo removal rates and the effect of noise on the proton beam core in case of a pulsed operation.

This note is organized in the following chapter: In Sec. II an overview of the different HL-LHC scenarios considered in the simulations is given and in Sec. III the optics at the two HEL positions currently considered are compared. The halo removal rates for the different scenarios and a DC operation of the HEL are compared in Sec. IV and the impact of a pulsed operation is discussed in Sec. V. For a validation of the beam-beam scenarios and the leveling, the minimum dynamic aperture has been compared as it is expected 
to drop down to very low values of even $2-3 \sigma$ in the presence of octupoles and high chromaticity. These values are unrealistic and thus a chromaticity of 3 has been chosen instead. The results of the study are summarized in Appendix A. For all scenarios studied the frequency map analysis (FMA) plots can be found in Appendix C 1-C 2.

The effects on the core are not treated in this note. A good summary in preparation of recent experiments is given in [6].

\section{HL-LHC SCENARIOS}

The main application of the hollow electron will be at top energy, explicitly at flat top and during the squeeze. In order to include later also the machine non-linearities, the layout version HLLHCV1.0 has been used, which is the latest version available including the latest error tables, correction routines and beam-beam elements. The changes compared to the latest layout (HLLHCV1.2) and the new layout (HLLHCV1.3) are expected to be small in terms of expected performance of the HEL as the changes in dynamic aperture (DA) and optics in IR4 for the different layouts stay small (to be confirmed for HLLHCV1.3) ${ }^{1}$.

Table I. HL-LHC key scenarios at $7 \mathrm{TeV}$ to evaluate the performance of the HEL in terms of halo removal rates. In IP2 and IP8 $\beta^{*}$ is equal in both planes. In IP1 and IP5 the $\beta^{*}$ is different in $x$ and $y$ for flat optics. For the crossing and separation the half crossing angles and half separation are stated. The octupole current listed is the current of the focusing octupole family (MOF circuit). For the scenarios with zero separation and beam-beam interaction full crabbing is assumed including four crab cavities per side and per IP.

\begin{tabular}{|c|c|c|c|c|c|c|c|}
\hline LHC cycle & $\begin{array}{c}\beta_{x, y}^{*}[\mathrm{~m}] \\
\mathrm{IP} 1 / 5\end{array}$ & $\begin{array}{c}\beta^{*}[\mathrm{~m}] \\
\mathrm{IP} 2 / 8\end{array}$ & $\begin{array}{c}\mathrm{x} \text {-angle }[\mu \mathrm{rad}] \\
(\mathrm{IP} 1 / 2 / 5 / 8)\end{array}$ & $\begin{array}{c}\text { spectrometer } \\
\text { polarity (IP2/8) }\end{array}$ & $\begin{array}{c}\text { separation }[\mathrm{mm}] \\
\text { IP1/2/5/8 }\end{array}$ & $Q_{x / y}^{\prime}$ & $I_{\mathrm{MO}}[\mathrm{A}]$ \\
\hline \multirow{3}{*}{ flat top } & \multirow{3}{*}{$6.0,6.0$} & \multirow{3}{*}{$10.0 / 3.0$} & \multirow{3}{*}{$295 / 170 / 295 / 220$} & \multirow{3}{*}{$1 / 1$} & \multirow{3}{*}{$0.75 / 2.0 / 0.75 / 2.0$} & 15 & -550 \\
\hline & & & & & & 3 & -550 \\
\hline & & & & & & 3 & 0 \\
\hline start leveling & $0.7,0.7$ & $10.0 / 3.0$ & $295 / 170 / 295 / 220$ & $1 / 1$ & $0 / 0 / 0 / 0$ & 3 & -550 \\
\hline squeezed round & $0.15,0.15$ & $10.0 / 3.0$ & $295 / 170 / 295 / 220$ & $1 / 1$ & 0/0/0/0 & 3 & -550 \\
\hline squeezed flat & $0.075,0.30$ & $10.0 / 3.0$ & $275 / 170 / 275 / 220$ & $1 / 1$ & 0/0/0/0 & 3 & -550 \\
\hline
\end{tabular}

The identified key scenarios are summarized in Table I and are based on [7] with updated values for the octupole current and chromaticity [8]. For the presented scenarios it is assumed that the beams are squeezed while colliding from $\beta_{\mathrm{IP} 1 / 5}^{*}=6 \mathrm{~m}$ to $0.7 \mathrm{~m}$. As higher removal rates are expected for colliding beams, the case without collisions is chosen in order to give a lower limit for the expected removal rates before the squeeze. As a high chromaticity and high octupole current in general also increases the halo removal rate,

\footnotetext{
${ }^{1}$ In general a decrease in DA will result in higher halo removal rates and larger effect of HEL imperfections on the core. A change in optics in IR4 is only relevant if the ratio of the beam size at the location of the HEL changes, explicitly if the proton beam diverges from a round shape. In this case, the HEL would deplete the halo down to smaller amplitudes in the plane with the larger beam size than the one with the smaller beam size. For details see also Sec. IV B.
} 
Table II. HL-LHC leveling scenario for squeezed collision optics with $\beta_{x / y}^{*}=0.15 / 0.15 \mathrm{~m}$. The beta-function in IP2 and IP8 stays constant.

\begin{tabular}{ll}
\hline \multicolumn{3}{|l}{$(\mathrm{IP} 1 / 5)[\mathrm{m}]$} & bunch intensity $\left[10^{11}\right]$ \\
\hline \hline $0.70,0.70$ & 2.2 \\
\hline $0.42,0.42$ & 1.7 \\
\hline $0.30,0.30$ & 1.5 \\
\hline $0.15,0.15$ & 1.1 \\
\hline
\end{tabular}

the scenario with low chromaticity and zero octupole current has been simulated in addition in order to study the influence of both factors.

The luminosity is then leveled between $\beta_{\mathrm{IP} 1 / 5}^{*}=0.7 \mathrm{~m}$ and the final squeezed optics, here round collision optics with $\beta^{*}=0.15 / 0.15 \mathrm{~m}$ and flat collision optics with $\beta^{*}=0.075 / 0.30 \mathrm{~m}$. The used leveling scenario is described in Table II. For a first evaluation of the HEL it is sufficient to simulate the start and end of the leveling of the leveling as these two points represent the cases of maximum and minimum DA during the leveling ${ }^{2}$. For the case of round optics the DA has been studied for the leveling scenario described in Table II including long-range and head-on beam-beam interactions with full crabbing and the LHCb spectrometer polarity yielding the smallest DA. The results of the DA simulation are summarized in Appendix A.

\section{HEL POSITION AND OPTICS IN IR4}

It is in general possible to generate an round and elliptical hollow electron beam, however one is then restricted to this shape as the shape is defined by the geometry of the cathode. A round shape is preferable over an elliptical shape as in case of an elliptical shape the ellipse will change shape while propagating through the e-lens due to the space-charge forces. Therefore we consider the electron beam to be round in this paper.

For an optimal and equal cleaning in both planes, the HEL is thus best installed at a location with equal $\beta$-functions. This condition is fulfilled for the nominal LHC optics at a location of $\pm 40 \mathrm{~m}$ from IP4 and is also approximately true for the HL-LHC optics. However, for the current HL-LHC layout this location is already occupied and an alternative location at $\pm 88.6 \mathrm{~m}$ is considered. At this location the $\beta$-functions are not equal, which also provides the possibility to study the differences between the optimal case of a round e-beam and round p-beam and the less optimal case of a round e-beam but elliptical p-beam. The $\beta$-function and $\beta$-function ratio for both locations is listed in Table III. For all HL-LHC scenarios the Twiss parameters at the HEL location are the same as at for all optics the Twiss parameters at IP4 are matched to the same value and the HEL and IP4 are only separated by a drift space for both locations. The optics in

\footnotetext{
${ }^{2}$ Here we make the assumption that the halo removal rates are correlated with the dynamic aperture. This is a valid assumption to narrow down the scenarios to be studied. However, no scaling law or similar, if it exists, has been established between the halo removal rate and the dynamic aperture and therefore the obtained values represent only rough boundary values for the halo removal rates throughout the squeeze.
} 
Table III. $\beta$-function and $\beta$-function ratio for the two HEL positions at $\pm 40 \mathrm{~m}$ and $\pm 88.6 \mathrm{~m}$. The Twiss parameters at the HEL are the same for all scenarios considered in this paper as the Twiss parameters at IP4 are matched to the same value for all scenarios and the HEL is only separated by a drift space from IP4. For the ratio $\beta_{x} / \beta_{y}$ the larger value is always ivided by the smaller one. The best ratios are indicated in bold.

\begin{tabular}{|c|c|c|c|c|c|c|c|c|c|c|}
\hline \multirow[t]{2}{*}{$\operatorname{pos}[\mathrm{m}]$} & $\beta_{x}$ & [m] & $\beta_{y}[$ & [m] & ratio & $\beta_{x} / \beta_{y}$ & $D_{x}$ & {$[\mathrm{~m}]$} & $D_{y}$ & [m] \\
\hline & \multicolumn{10}{|c|}{ beam 1 beam 2 beam 1 beam 2 beam 1 beam 2 beam 1 beam 2 beam 1 beam 2} \\
\hline-40.00 & 231.64 & 194.57 & 211.94 & 356.79 & 1.09 & 1.83 & 0.00 & 0.00 & 0.00 & 0.00 \\
\hline 40.00 & 138.73 & 280.60 & 264.02 & 262.58 & 1.90 & 1.07 & 0.00 & 0.00 & 0.00 & 0.00 \\
\hline-88.60 & 336.44 & 177.55 & 210.32 & 441.84 & 1.60 & 2.49 & -0.06 & 0.06 & 0.00 & 0.00 \\
\hline 88.60 & 130.65 & 368.11 & 325.67 & 233.17 & 2.49 & 1.58 & -0.06 & 0.06 & 0.00 & 0.00 \\
\hline
\end{tabular}

IR4 is shown in Fig. 1 with both HEL locations indicated by dashed lines.
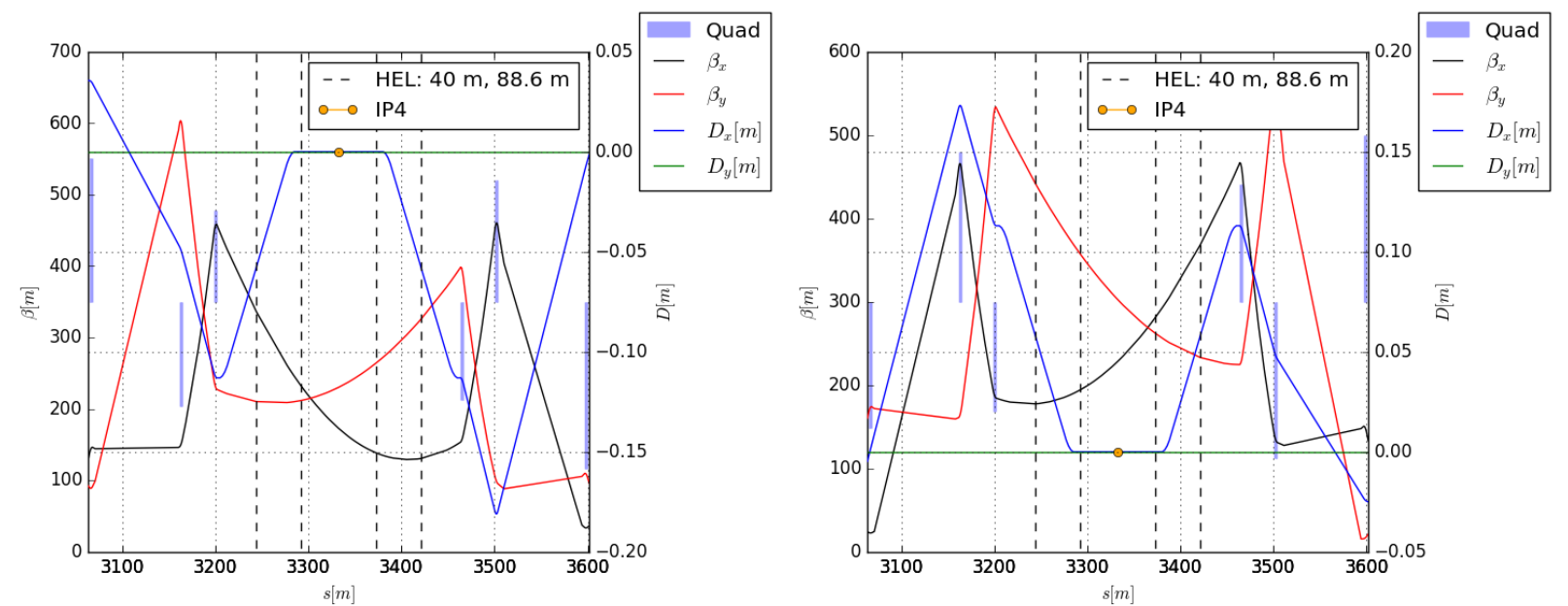

Figure 1. Optics in IR4 for flat top scenario with $\beta^{*}(\mathrm{IP} 1 / 2 / 5 / 8)=6.0 / 10.0 / 6.0 / 3.0$ and Beam 1 (left) and Beam 2 (right). The different HEL locations are indicated with dashed lines and IP4 with one orange point.

\section{HALO REMOVAL RATES FOR THE DIFFERENT HL-LHC SCENARIOS AND DC OPERATION}

In order to estimate the halo removal rates for the different HL-LHC key scenarios identified in Sec. II, tracking simulations with LifeTrac [9] with the following parameters have been performed:

- distribution: uniform distribution in $x$ and $y$ between $4-6 \sigma$ and $x^{\prime}=y^{\prime}=0$ with

- $\frac{\Delta p}{p_{0}}==0$ for all particles in order to study the behavior of on-momentum particles

- Gaussian distribution in $(z, \delta p)$ cut at $6 \sigma$ in order to study the effect on off-momentum particles 
- number of particles: $10^{4}$

- number of turns: $10^{6}$

- beam parameters: normalized emittance $\varepsilon_{x, y}=2.5 \mu \mathrm{m}$, momentum spread $\frac{\Delta p}{p_{0}}=1.1 \times 10^{-4}, 1 \sigma$ bunch length $\sigma_{s}=7.5 \mathrm{~cm}$

- only Beam 1 is tracked

- collimation: one black absorber with 6 beam $\sigma$ opening installed at IP3.

- HEL parameters: HEL installed at $-40 \mathrm{~m}$ from IP4 with the inner radius $r_{1}$ adjusted to $4 \sigma$. The remaining parameters are taken from [2] and are based on the current design of the HEL gun, explicitly: $E_{\mathrm{p} \text {-beam }}=7 \mathrm{TeV}, E_{\mathrm{e}-\text { beam }}=10 \mathrm{keV}$, length of HEL $l=3 \mathrm{~m}$, inner radius $r_{1}=4 \cdot \max \left(\sigma_{x}, \sigma_{y}\right)$, ratio outer to inner radius $r_{2} / r_{1}=1.8815$. For the electron beam current three different values are simulated:

1. $I_{\mathrm{e}-\text { beam }}=3.6 \mathrm{~A}$ : The HEL design report value, which is also used for the specification of the current gun [2]

2. $I_{\mathrm{e}-\text { beam }}=5.0 \mathrm{~A}$ : maximum current achievable by the current e-gun.

- HEL profile: an ideal uniform profile between $r_{1}$ and $r_{2}$ is assumed as electron beam profile. The radial kick is then given by: described by a shape function $f(r)$ and a maximum kick strength $\theta_{\max }$ :

$$
\theta(r)=\frac{f(r)}{\left(r / r_{2}\right)} \cdot \theta_{\max }
$$

with $r=\sqrt{x^{2}+y^{2}}$ and $\theta_{\max }$ independent of $r$. For a uniform profile one then obtains:

$$
f(r)=\left\{\begin{array}{lll}
0 & , \quad r<r_{1} \\
\frac{r^{2}-r_{1}^{2}}{r_{2}^{2}-r_{1}^{2}} & , \quad r_{1} \leq r<r_{2} \\
1 & , \quad r_{2} \leq r
\end{array}\right.
$$

and

$$
\theta_{\max }=\theta\left(r_{2}\right)=\frac{2 L I_{T}\left(1 \pm \beta_{e} \beta_{p}\right)}{4 \pi \varepsilon_{0}(B \rho)_{p} \beta_{e} \beta_{p} c^{2}} \cdot \frac{1}{r_{2}}
$$

in the case that electron and proton beam are traveling in the same direction with $L$ the length of the e-lens, $I_{T}$ the total electron beam current, $\beta_{e}$ and $\beta_{p}$ the relativistic $\beta$ of electron and proton beam, $B \rho$ the magnetic rigidity, $c$ the speed of light and $\varepsilon_{0}$ the vacuum permittivity. 


\section{A. Halo removal rates at flat top for HEL installed at $\mathbf{- 4 0} \mathrm{m}$ from IP4 and different chromaticity and octupole settings}

In order to compare the simulation results to previous simulations, the scenario considered in [3, 4] nominal LHC, $\beta^{*}=0.50 \mathrm{~m}, Q_{x / y}^{\prime}=2.0 / 2.0, I_{\mathrm{MO}}=0 \mathrm{~A}$, no collisions, no errors, no crossing and separation - is compared to the HL-LHC flat top scenario with different chromaticity and octupole settings. The halo removal rates are shown in Fig. 2. To better understand the results, the results of the FMA analysis for all
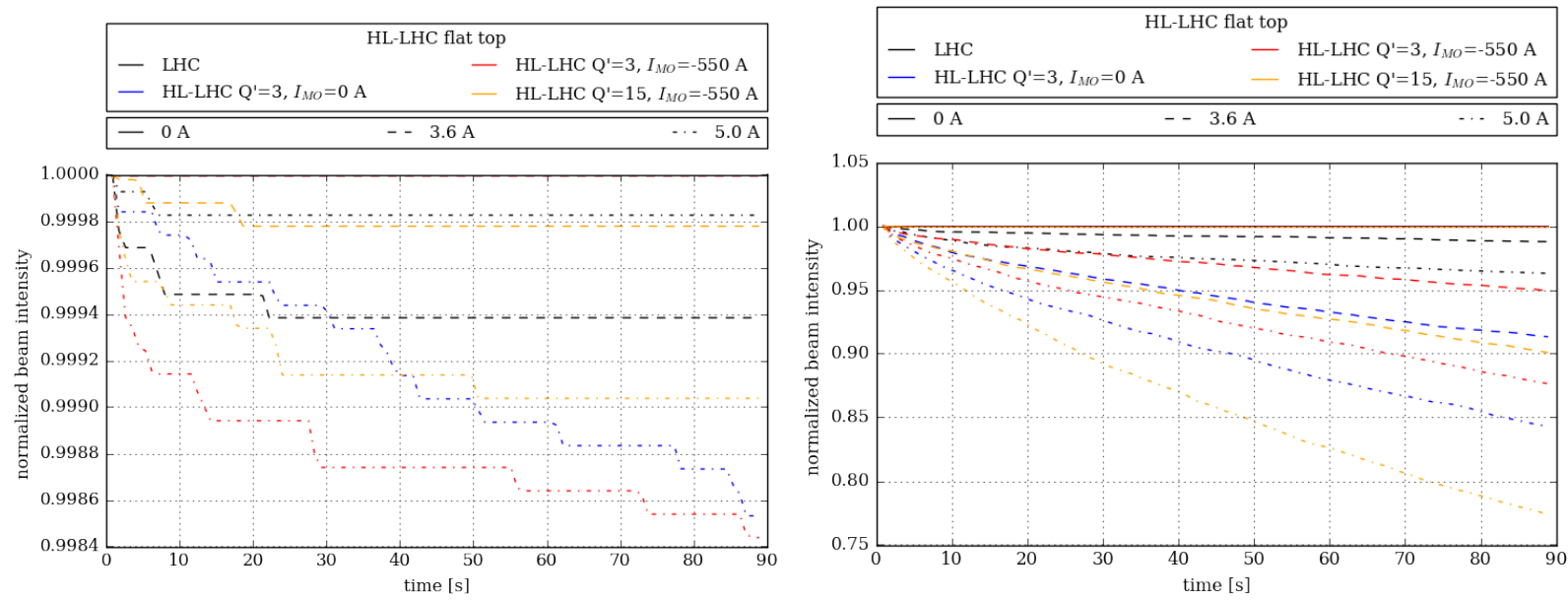

Figure 2. Halo removal rates at flat top $\left(\beta^{*}(\operatorname{IP} 1 / 2 / 5 / 8)=6.0 / 10.0 / 6.0 / 3.0\right.$ for different chromaticity and octupole settings. For on-momentum with $\frac{\Delta p}{p_{0}}=0$ for all particles (left) the halo removal rates are very small for all cases. For a Gaussian distribution in $\left(z, \frac{\Delta p}{p_{0}}\right)$ (right), the halo removal rates increase considerably with an increase in chromaticity.

cases are summarized in Appendix C 1 a. Several observations can be made:

- The HEL itself introduces detuning with amplitude, but only for particles between 4 and $6 \sigma$ (see Appendix $\mathrm{C} 1 \mathrm{a}$ ). This leads to dominant detuning with amplitude from the HEL for $Q^{\prime}=3, I_{\mathrm{MO}}=$ $0 \mathrm{~A}$ and a folding of the tune footprint in the presence of octupoles, explicitly for $Q^{\prime}=3, I_{\mathrm{MO}}=$ $-550 \mathrm{~A}$ and $Q^{\prime}=15, I_{\mathrm{MO}}=-550 \mathrm{~A}$.

- for on-momentum the halo removal rates do not consistently scale with the HEL current (see Fig. 2 left), e.g. for $Q^{\prime}=3, I_{\mathrm{MO}}=-550 \mathrm{~A}$ and 3.6 A HEL current no losses are observed, while for the same case and 5.0 A the largest losses are observed. Furthermore, for the nominal LHC the losses for 3.6A HEL current are higher than for 5.0 A HEL current. For all cases the losses are extremely small, meaning that basically single particles are lost. The inconsistent behavior is therefore just due to lack of statistics.

- for off-momentum the halo removal rates are surprisingly larger for $Q^{\prime}=3, I_{\mathrm{MO}}=0$ A than for $Q^{\prime}=3, I_{\mathrm{MO}}=-550 \mathrm{~A}$. This result might however be very sensitive to the choice of working point as the tune spread is so small and the halo removal rate thus probably depend on the excitation of a 
few high order resonances. The largest halo removal rates are obtained for $Q^{\prime}=15, I_{\mathrm{MO}}=-550 \mathrm{~A}$ consistent with the picture that stronger non-linearities lead to higher halo removal rates.

- the halo removal rates for off-momentum scale with the HEL current (Fig. 2 right). This is not the case for on-momentum, most likely due to a lack of statistics (see first point).

- the combination of the detuning with amplitude from the octupoles and the HEL leads to a double folding of the tune footprint. This is illustrated in Fig. 3 where the tune footprint for a circular grid up to $4.4,8.0$ and $10.0 \sigma$ is shown.

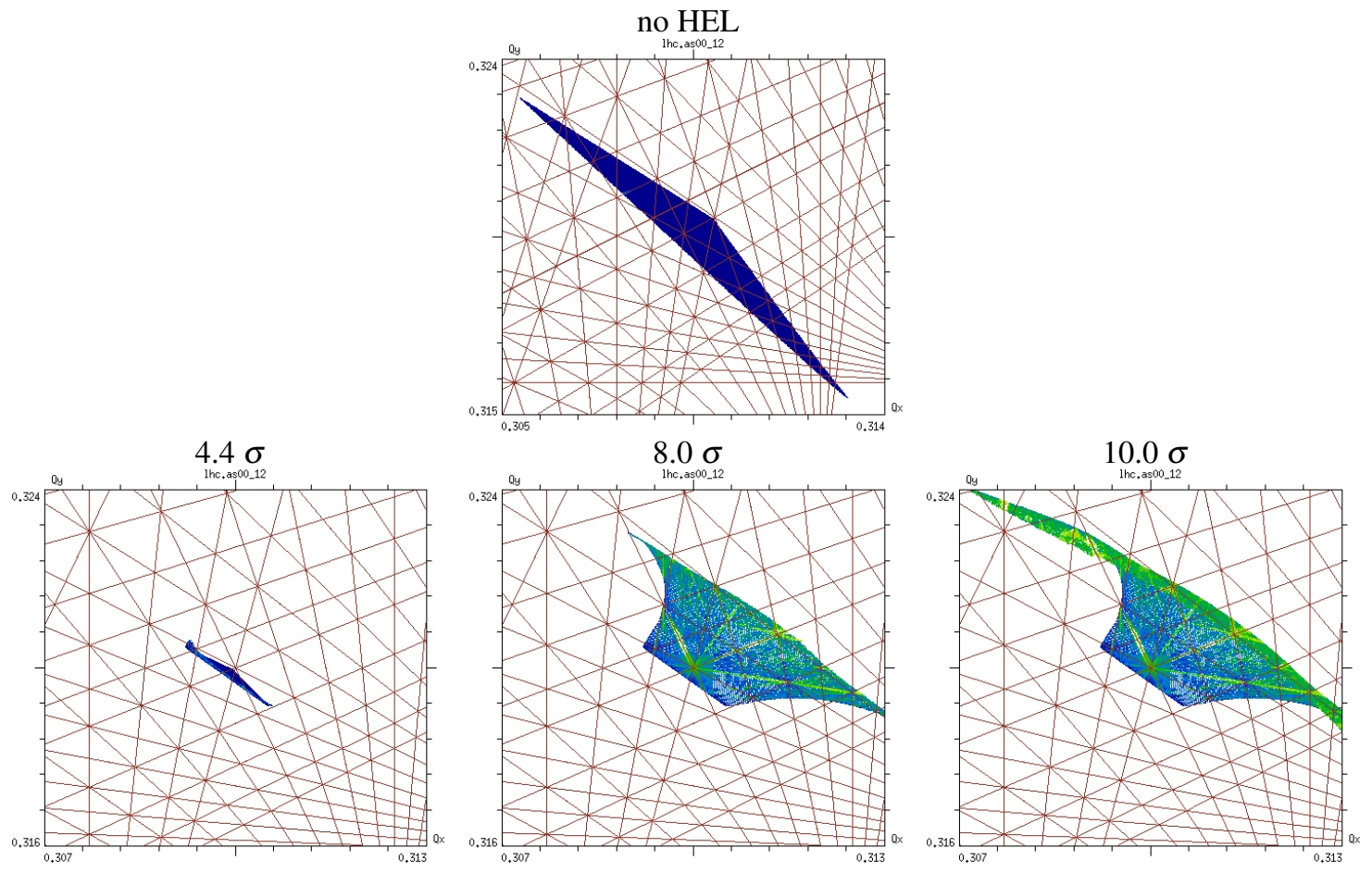

Figure 3. flat top, $Q^{\prime}=15, I_{\mathrm{MO}}=-550 \mathrm{~A}$, HEL installed at $-40 \mathrm{~m}$ : FMA analysis for on-momentum particles $\left(\frac{\Delta p}{p_{0}}=0.0 \sigma_{p}\right)$ up to 4.4, 8.0 and $10 \sigma$ amplitude for a circular grid with HEL for $3.6 \mathrm{~A}$ and up to $10 \sigma$ without HEL. The resonance lines up to 20th order are indicated in red. The combination of the HEL and the octupoles leads to a double folding of the tune footprint. 


\section{B. Halo removal rates at flat top in the presence of errors and for HEL installed at $\mathbf{- 4 0} \mathrm{m}$ or $\mathbf{- 8 8 . 6} \mathrm{m}$ from IP4}

To study the effect of machine errors on the HEL performance, the standard errors as used usually in DA simulations have been assigned and the results for the case without errors compared with the case with errors for seed 1. Explicitly the following errors have been assigned:

- all $a_{n}, b_{n}$ errors up to 14 th order, except $a_{1}, b_{1}$, and $b_{2}$ errors. The $a_{1}, b_{1}$, are set to zero as the orbit correction with micado often fails. The current $b_{2}$ errors lead to larger beta-beat. As the beta-beat is currently not corrected in the simulation input files, this error is also set to zero in order to avoid any unrealistic dependence.

- nominal LHC error tables and new IT,D1,D2,Q4 and Q5 error tables for HL-LHC, explicitly ITa_errortable_v5, ITb_errortable_v5, D1_errortable_v1_spec, D2_errortable_v5_spec, Q4_errortable_v2_spec, Q5_errortable_v0_spec

In order to artificially introduce stronger coupling, the coupling correction is stopped before the empirical optimization (parameter "closest1"), which leads to $Q_{x}-Q_{y}=2.0 \times 10^{-5}$ for seed 1 . The motivation behind increasing the coupling between the two planes is to study the behavior of the halo removal rates for unequal $\beta$-functions in both planes as it is the case for the HEL at $-88.6 \mathrm{~m}$ from IP4. The hypothesis is that in the presence of coupling, the planes mix and the reduction due to the unequal $\beta$-functions is mitigated or at least reduced. The halo removal rates for the four different cases are shown in Fig. 4. The following observations
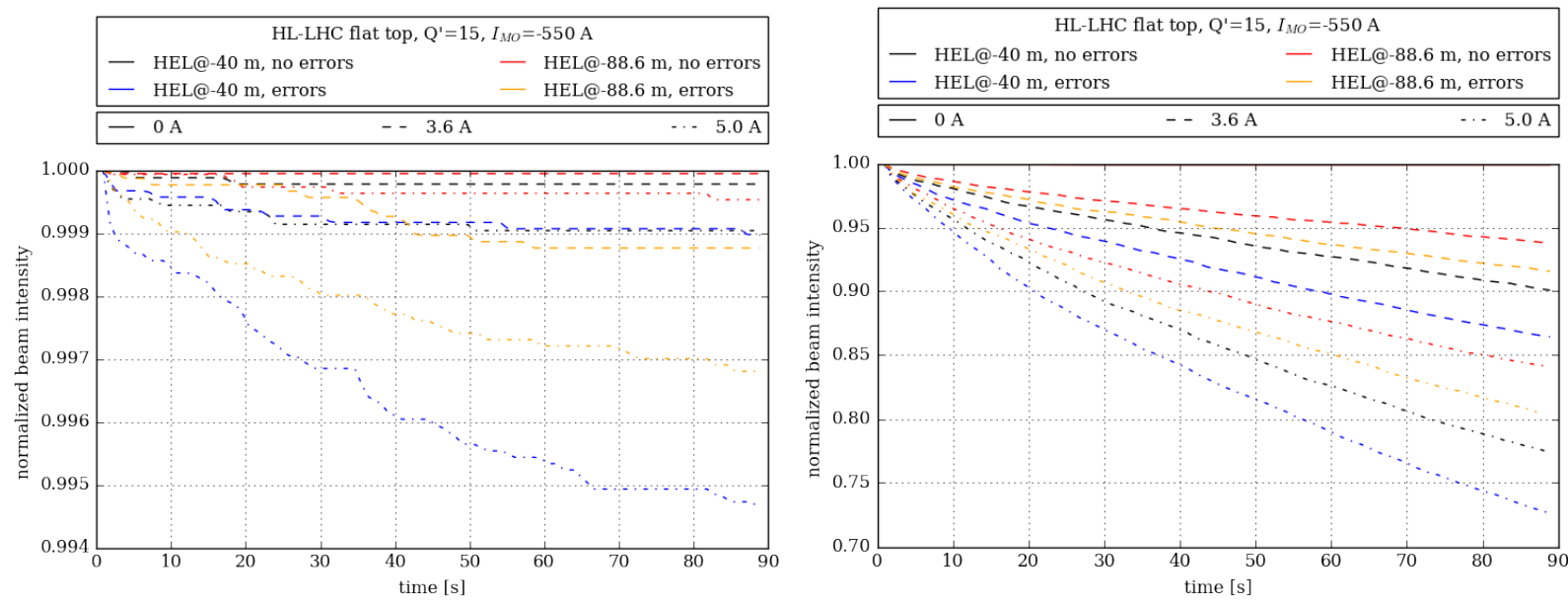

Figure 4. Halo removal rates at flat top $\left(\beta^{*}(\operatorname{IP} 1 / 2 / 5 / 8)=6.0 / 10.0 / 6.0 / 3.0\right.$ for for the case with and without magnetic errors and for the HEL installed at $-40 \mathrm{~m}$ from IP4 and $-88.6 \mathrm{~m}$ from IP4. For on-momentum with $\frac{\Delta p}{p_{0}}=0$ for all particles (left) and for a Gaussian distribution in $\left(z, \frac{\Delta p}{p_{0}}\right)$ (right), the halo removal rates increase for the case with errors an decrease for the HEL installed at $-88.6 \mathrm{~m}$ from IP4.

can be made:

- The machine errors lead to a considerable increase in the halo removal rate for on and off-momentum. 
- The halo removal rates increase with the HEL current.

- The coupling introduced in the simulations does not lead to a mitigation of the reduction of the halo removal rate in the case of unequal $\beta$-functions at the location of the HEL (here for the position of $-88.6 \mathrm{~m}$ from IP4) as the coupling is too small in order to lead to a sufficient mixing of the plane. Comparing the FMA plots with and without errors, also no mixing of the planes e is observed being equivalent with an increase in the diffusion in tune in the plane with the smaller beam size, here the vertical plane.

The FMA analysis for these scenarios are shown in Appendix $\mathrm{C} 1 \mathrm{~b}$. 


\section{Halo removal rates during $\beta^{*}$ leveling for HEL installed at $\mathbf{- 4 0} \mathrm{m}$ from IP4}

For $\beta^{*}$ leveling the scenario described in Table II is used as baseline. To get an estimate for the halo removal rates throughout the leveling, the following cases have been studied (for details see Sec. II), which represent the cases of minimum and maximum DA during the leveling:

1. start of leveling: $\beta_{x / y}^{*}=0.7 / 0.7 \mathrm{~m}$,

2. end of squeeze:

(a) $\beta_{x / y}^{*}=0.15 / 0.15 \mathrm{~m}$ (round optics),

(b) $\beta_{x / y}^{*}=0.075 / 0.30 \mathrm{~m}$ (flat optics).

For all cases full crabbing and all long-range beam-beam encounters are considered. The halo removal rates for all cases are shown in Fig. 5. To better understand the results, the results of the FMA analysis for all cases is summarized in Appendix C2. Several observations can be made:
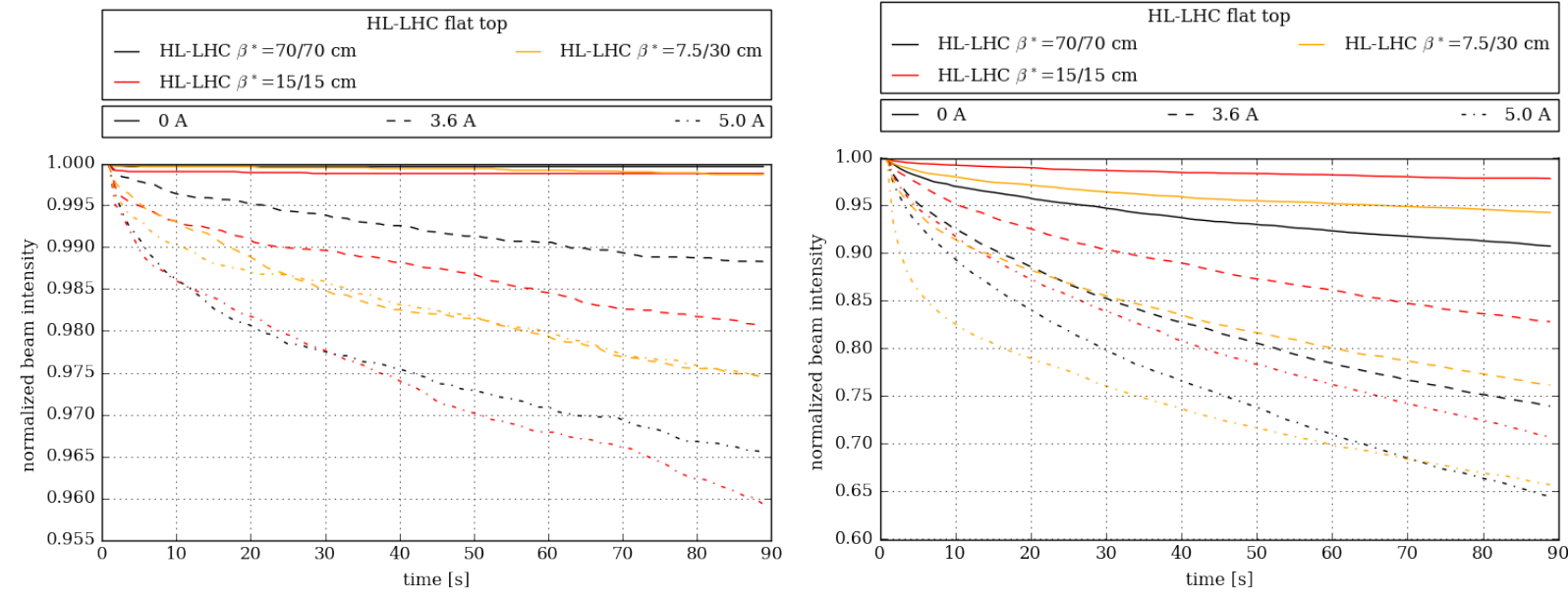

Figure 5. Halo removal rates during $\beta^{*}$ leveling. For on-momentum with $\frac{\Delta p}{p_{0}}=0$ for all particles (left) the halo removal rates are small for all cases. For a Gaussian distribution in $\left(z, \frac{\Delta p}{p_{0}}\right)$ (right), the halo removal rates increase with the HEL current and are largest for $\beta_{x / y}^{*}=0.075 / 0.30 \mathrm{~cm}$.

- In contrast to the case at flat top, losses are already present for the case without HEL - small losses for on-moment (Fig. 5 left, solid line) and considerable losses for off-momentum (Fig. 5 right, solid line). The highest losses are observed for off-momentum at $\beta_{x / y}^{*}=70 / 70 \mathrm{~cm}$ in agreement with the results of the DA simulations ${ }^{3}$.

\footnotetext{
${ }^{3}$ Note that the DA simulations are always conducted for $\frac{\Delta p}{p_{0}}=2.7 \times 10^{-4}$ which corresponds to $2.4 \sigma$ in $\frac{\Delta p}{p_{0}}$
} 
- The particles amplitudes for which the HEL is active, explicitly the amplitudes between 4 and $6 \sigma$, lie precisely between the point where the tune footprint converges in one point and then later folds back (see Fig. 6).

- In all cases, the HEL increases the diffusion rate further and the diffusion increases with the HEL current, except in the case of $\frac{\Delta p}{p_{0}}=0$ and $\beta_{x / y}^{*}=0.075 / 0.30 \mathrm{~cm}$ where the removal rates are almost the same.
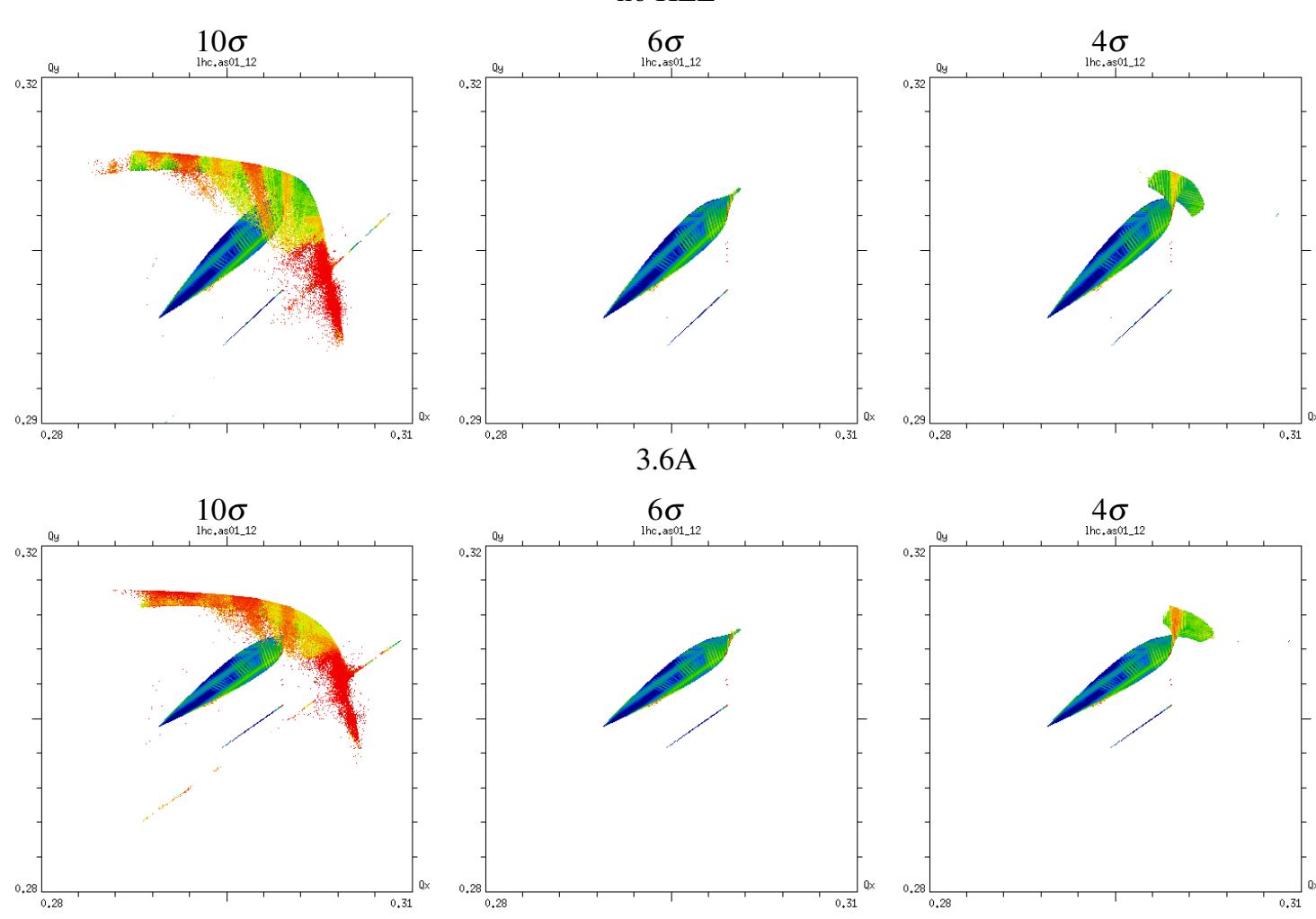

Figure 6. $\beta^{*}$-leveling, $\beta_{x / y}^{*}=7.5 / 30.0 \mathrm{~cm}, Q^{\prime}=3, I_{\mathrm{MO}}=-550 \mathrm{~A}$, HEL installed at $-40 \mathrm{~m}$ : FMA analysis for offmomentum particles $\left(\frac{\Delta p}{p_{0}}=0.1\right)$ up to $10 \sigma, 6 \sigma$ and $4 \sigma$ amplitude for a circular grid with and without HEL.

\section{HALO REMOVAL RATES FOR THE DIFFERENT HL-LHC SCENARIOS AND PULSED OPERATION}

One of the motivation for the HEL are the mitigation of the loss spikes observed during the squeeze in 2012 [? ]. With a fast depletion of the tails with the HEL before the start of the squeeze (at flattop), these loss spikes could be mitigated or at least considerably decreased. This implies, that at flat top a high halo removal rate is needed in order to clean the tails in a sufficiently small time. The simulations presented in this section show that the halo removal rates are however small in the case of separated beams (see 
Sec. IV A-IV B), in particular for low chromaticity and therefore a pulsing of the e-lens is considered in order to increase the halo removal rate. Two different pulsing patterns are considered:

- random: the e-beam current is modulated randomly,

- resonant: the e-lens is switched on only every $n$th turn with $n=2,3,4, \ldots$.

In case of a resonant excitation the halo removal rate strongly depends on the pulsing pattern as an excitation every $n$th turn in general drives $n$th order resonances [6]. Both pulsing patterns have been studied for the flat top scenario with $Q^{\prime}=15, I_{\mathrm{MO}}=-550 \mathrm{~A}$. In case of the random excitation a uniform random distribution between zero and the maximum HEL current is assumed. For the resonant excitation, the maximum kick is applied every $n$th turn. The obtained halo removal for random pulsing are shown in Fig. 7 and for resonant pulsing in Fig. 8 and the results of the FMA analysis are summarized in Appendix C $1 \mathrm{c}$.
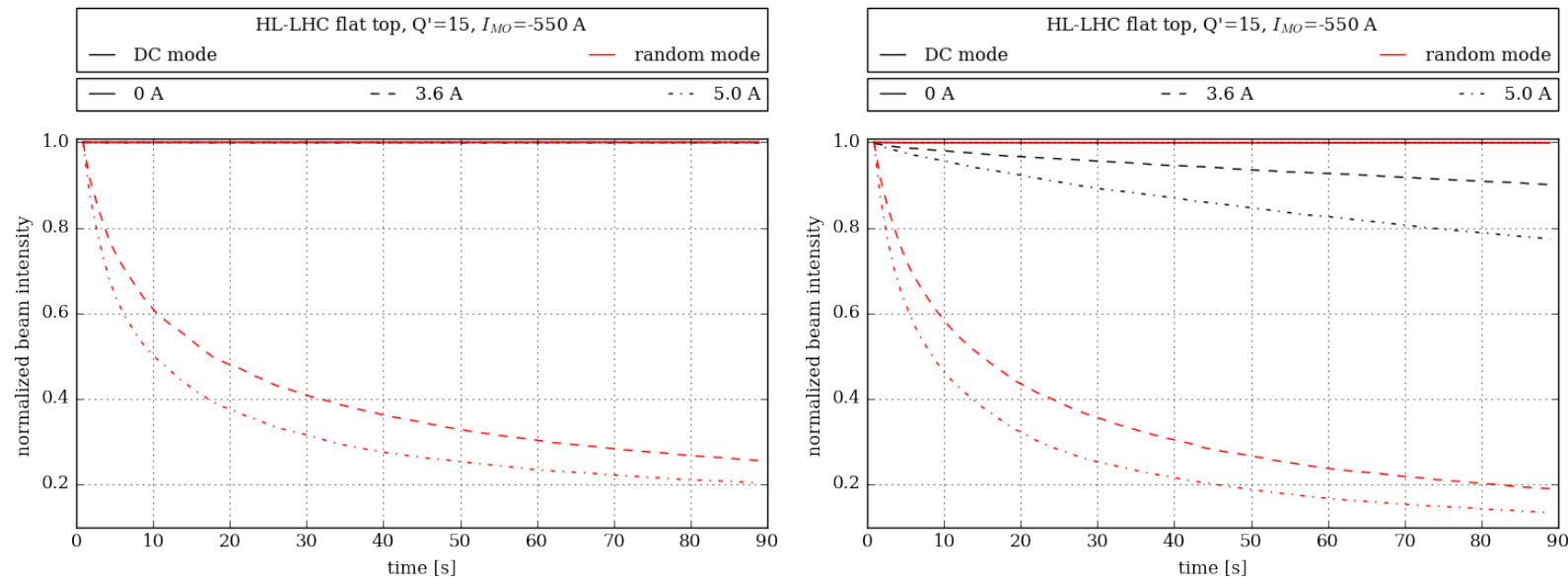

Figure 7. Halo removal rates at flat top $\left(\beta^{*}(\mathrm{IP} 1 / 2 / 5 / 8)=6.0 / 10.0 / 6.0 / 3.0\right)$ for $\mathrm{DC}$ and random pulsed operation. For on-momentum with $\frac{\Delta p}{p_{0}}=0$ for all particles (left) and for a Gaussian distribution in $\left(z, \frac{\Delta p}{p_{0}}\right)$ (right), the halo is almost entirely removed with the random pulsing.

The following observations can be made:

- The halo removal rate can be largely increased by pulsing the e-lens in random mode. Still to be shown is that the halo removal rate can be controlled by the amplitude of the current modulation.

- In the case of the random modulation the halo removal rate is so large, that only a small increase with the HEL current is observed. This might change for smaller modulation amplitudes.

- The halo removal rates for the resonant excitation are in general smaller than for the random excitation. For on-momentum pulsing every 2nd turn yields a larger halo removal rate than for the other pulsing patterns. This can be explained by the excitation of the $12 Q_{x}+4 Q_{y}=5$ resonance (see FMA 

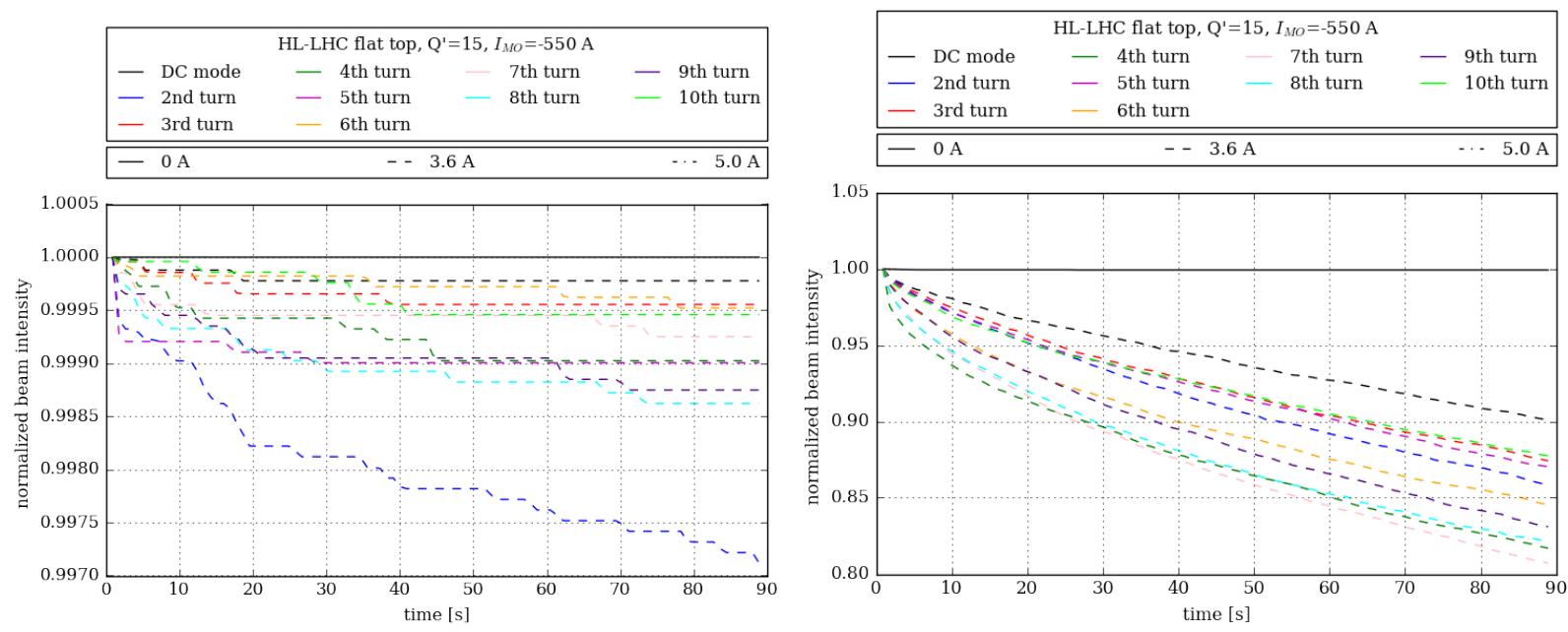

Figure 8. Halo removal rates at flat top $\left(\beta^{*}(\operatorname{IP} 1 / 2 / 5 / 8)=6.0 / 10.0 / 6.0 / 3.0\right)$ for DC and resonant pulsed operation. For on-momentum with $\frac{\Delta p}{p_{0}}=0$ for all particles (left) and for a Gaussian distribution in $\left(z, \frac{\Delta p}{p_{0}}\right)$ (right), the halo removal rate is increased due to the pulsing. Different halo removal rates are obtained for different pulsing patterns, where pulsing every 2nd turn is most efficient for on-momentum and pulsing every 4th or 7th turn for off-momentum.

analysis Appendix $\mathrm{C} 1 \mathrm{c}$ ). Due to the folding of the tune footprint, it is in general difficult to identify the driving resonances from the FMA analysis and no relation could be identified for the other pulsing patterns. All results from the FMA analysis are summarized in Appendix C $1 \mathrm{c}$. 


\section{MODELING OF THE HALO REMOVAL RATES}

For the simulations presented in this paper, a uniform distribution between $4-6 \sigma$ has been used. In this case, two regimes in the halo removal rate or relative intensity decay became apparent:

1. fast decay $=$ exponential regime: A fast exponential decay in the beginning

2. steady losses $=$ linear regime: Linear loss rate indicating a triangular shape of the distribution

This is is illustrated on the example of the different leveling cases in Fig. 9. We picked this scenario as it covers a wide range from weak to strong decays.

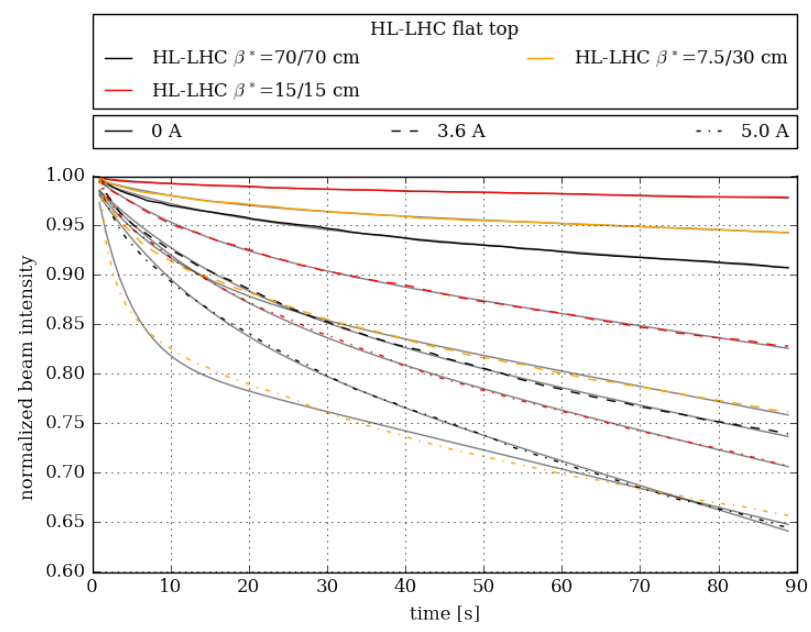

Figure 9. Halo removal rates during $\beta^{*}$ leveling off-momentum with a Gaussian distribution in $\left(z, \frac{\Delta p}{p_{0}}\right)$. The halo removal rate can be approximated with Eqn. 4. The resulting fits are indicated with solid gray lines.

The fit is indicated in gray where the following fit function has been used:

$$
I(t)=a+b \cdot t+A \cdot e^{-\frac{t}{\tau}}
$$

In particular the case with an e-lens current of 5.0 A for $\beta^{*}=0.075 / 0.30 \mathrm{~m}$ and $\beta^{*}=0.70 / 0.70 \mathrm{~m}$ is interesting as in the beginning the case with $\beta^{*}=0.075 / 0.30 \mathrm{~m}$ features a significantly more rapid decay than the case with $\beta^{*}=0.70 / 0.70 \mathrm{~m}$ which is then later reversed for the region of the steady losses. This behavior can be better understood with the help of the FMA analysis (Fig. 10). In the case of $\beta^{*}=0.075 / 0.30 \mathrm{~m}$, the HEL drives apparently a resonance leading to a strong diffusion in tune in the horizontal plane between $4-6 \sigma$ which in turn causes the strong exponential decay at the beginning. For $\beta^{*}=0.70 / 0.70 \mathrm{~m}$ the visible areas of high diffusion in tune between $4-6 \sigma$ are not considerably enhanced by the e-lens and there are not any particularly strong ones present in the first place. This then leads to a relatively continuous (linear) diffusion. In order to have a more quantitative comparison, this empirical fit model has been applied to all cases. The results are presented in the conclusion (Sec. VII) and a summary table in Appendix ??. 

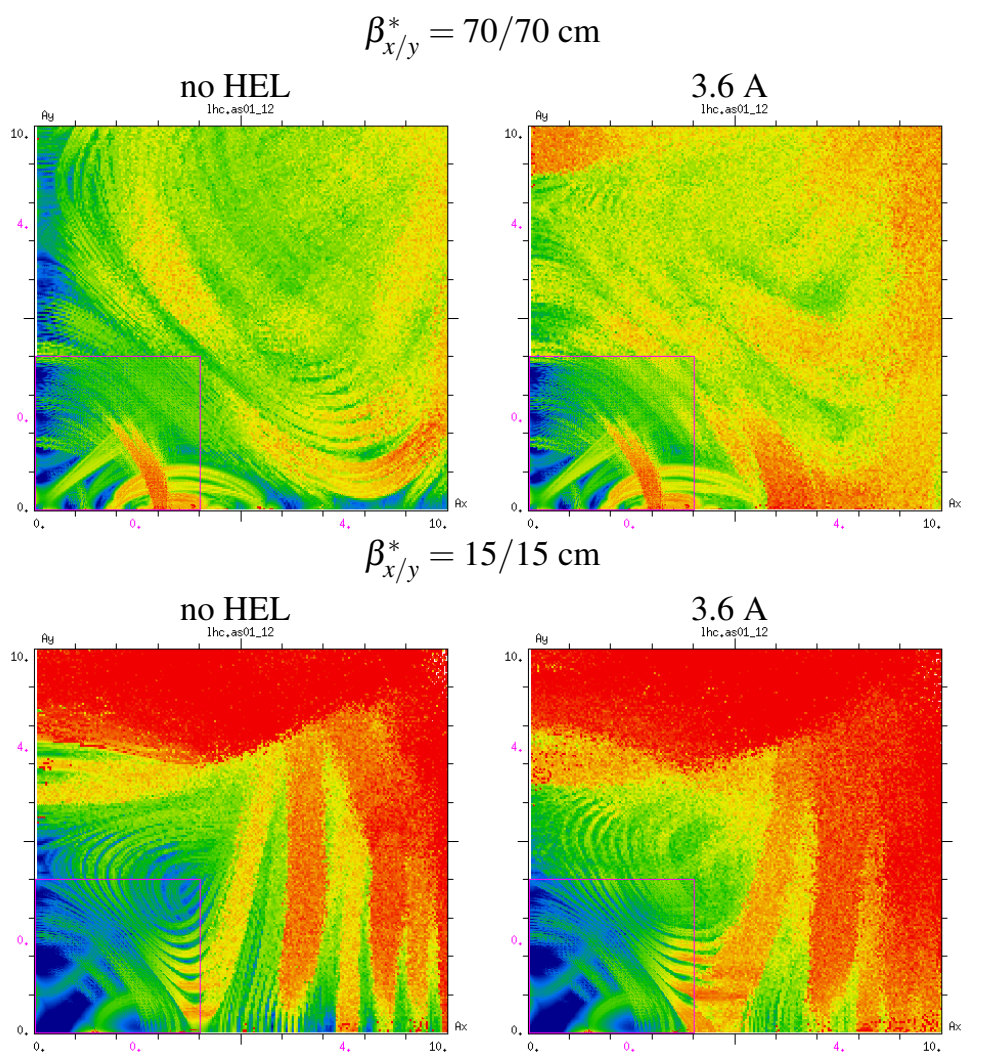

Figure 10. $\beta^{*}$-leveling, $\beta_{x / y}^{*}=70 / 70 \mathrm{~cm}$ (top) and $\beta_{x / y}^{*}=15 / 15 \mathrm{~cm}$ (bottom), $Q^{\prime}=3, I_{\mathrm{MO}}=-550 \mathrm{~A}$, HEL installed at $-40 \mathrm{~m}$ : FMA analysis in amplitude space for off-momentum particles $\left(\frac{\Delta p}{p_{0}}=0.1 \sigma_{p}\right)$ up to $10 \sigma$ amplitude for a quadratric grid with and without HEL.

In order to obtain the realistic halo removal rates, the real distribution needs to be simulated or the obtained halo removal rates for the uniform distribution would need to be folded with the real distribution. In addition diffusive processes like scattering processes and intra-beam scattering and synchrotron radiation for the damping effect need to be taken into account. However, a first comparison of the different scenarios can be already obtained with the model of the uniform halo also delivering information about at which amplitudes most particles are lost (see Appendix D). In particular in the case of different chromaticities also the longitudinal plane plays an import role and there might thus be a strong correlation between the momentum offset and the observed losses due to the large chromatic detuning. 


\section{CONCLUSIONS}

The aim of this note is to estimate the performance of the HEL for the different HL-LHC scenarios, where the key scenarios are summarized in Table I. The following main observations can be made:

- Halo removal rates for on-momentum particles are considerably smaller than for off-momentum. The only exception is the random excitation as the halo removal rate is so high in this case that it is similar for both cases (Sec. V).

- The halo removal rates at flat top are in general considerably smaller than during $\beta^{*}$-leveling due to the less pronounced non-linearities. Without HEL no losses are observed. The removal rates can then be increased between $0.01 \% / \mathrm{s}-0.13 \% / \mathrm{s}$ for a HEL current of $3.6 \mathrm{~A}$ and depending on the chromaticity and octupole settings (see Fig. 11).

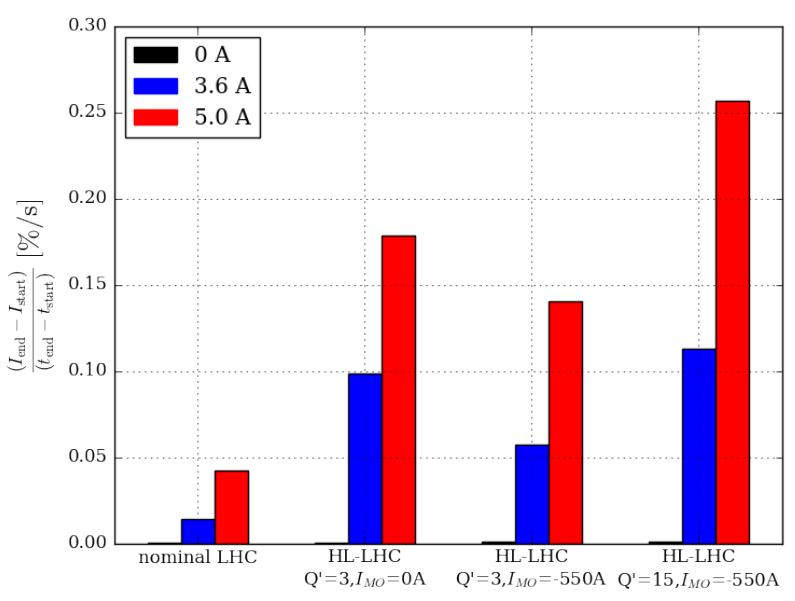

Figure 11. flat top and different chromaticity and octupole settings: Halo removal rates for the case of a uniform halo distribution between $4-6 \sigma$ transversely and a Gaussian distribution cut at $6 \sigma$ in the longitudinal plane. The removal rates are obtained by averaging the halo losses over $90 \mathrm{~s}$.

- During $\beta^{*}$-leveling minimal losses are already observed for on-momentum without HEL, but considerable losses are already present for a Gaussian distribution in the longitudinal plane (see Fig. 12) without HEL due to the interplay of the octupoles, beam-beam and chromaticity and the resulting small DA (Appendix A). Between 0.02\%/s-0.10\%/s are observed without HEL and for offmomentum. With HEL and a current of $3.6 \mathrm{~A}$, the halo removal rate increase to $0.01 \% / \mathrm{s}-0.03 \% / \mathrm{s}$ for on-momentum and $0.19 \% / \mathrm{s}-0.29 \% / \mathrm{s}$ for off-momentum.

- Non-linearities increase the halo removal rate. The increase depends on the resonances present. Except for rare cases the HEL appears to drive all resonances. Non-linearities considered and compared in this paper are: 

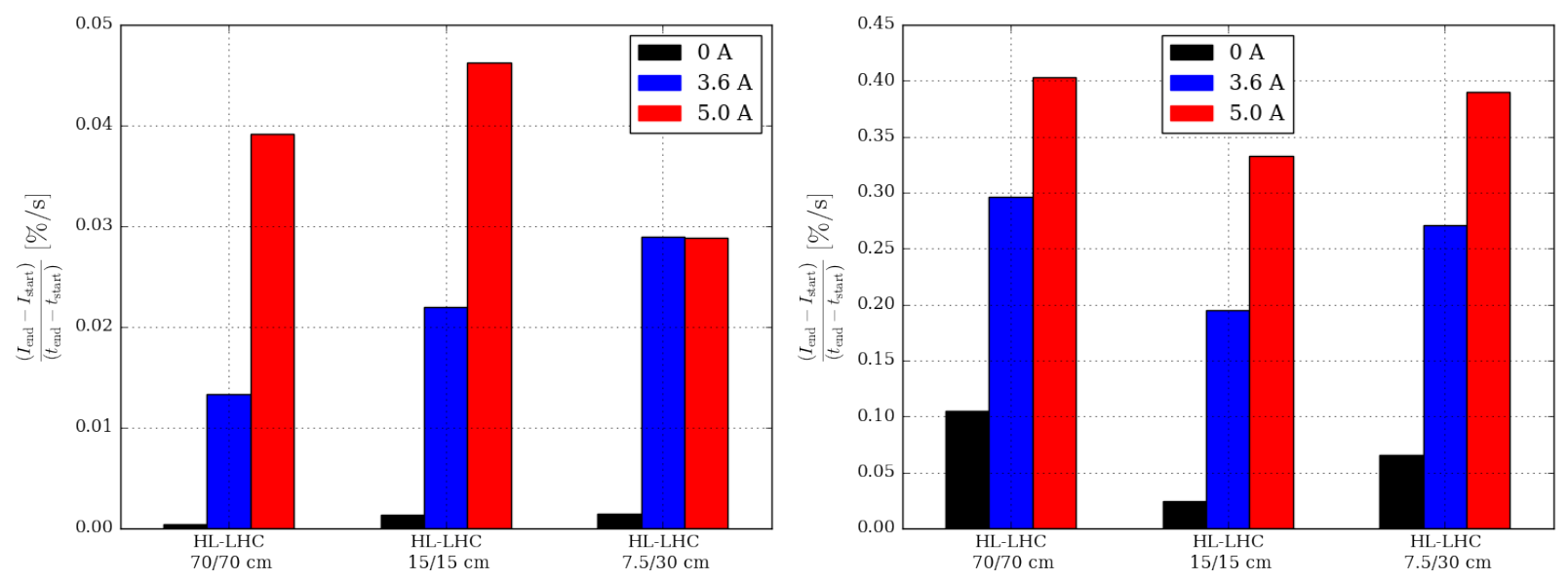

Figure 12. $\beta^{*}$ leveling: Halo removal rates for the case of a uniform halo distribution between $4-6 \sigma$ transversely and on momentum (left) and a Gaussian distribution cut at $6 \sigma$ in the longitudinal plane (right). The removal rates are obtained by averaging the halo losses over $90 \mathrm{~s}$.

1. Head-on and long-range beam-beam effects including crab cavities: Note that in this case losses are already present without HEL due to the very small dynamic aperture. The smallest DA is here observed for $\beta^{*}=0.70 / 0.70 \mathrm{~m}$ (see Appendix A). In the linear regime, the halo removal rates increase with the HEL current (fit parameter $b$ in Fig. 12). In the exponential regime the halo removal rates expressed in form of the decay time $\tau$ (fit parameter $\tau$ in Fig. 12) depends on the non-linearities present and it is difficult to observe a pattern.

2. Chromaticity and Landau Damping Octupoles: Both knobs are used in order to control instabilities during the fill. Both are also particularly relevant at flat top where the octupoles present the main source of detuning with amplitude. The combination of the HEL with the octupoles leads to a double folding of the tune footprint (Appendix C $1 \mathrm{a}$ ). The chromaticity also contributes considerably to the overall tune spread due to the chromatic detuning. In addition, a high chromaticity also implies a constant swiping of the particles over resonances due to the synchrotron motion.

The dependence of the halo removal rates on both parameters is illustrated in Fig. 11 in form of the two fit parameters $b$ and $\tau$. At flat top the linear regime is in general dominating and a clear scaling of the slope $b$ and thus the halo removal rate with the HEL current is visible. Higher octupole current alone does not seem to increase the halo removal rate. An increase of the chromaticity leads to a considerable increase. It should be also noted that in the case of low chromaticity and zero octupole current $\left(Q^{\prime}=3, I_{r m M O}=0\right)$ the observed loss rates become extremely sensitive to the chosen working point as the tune tune spread is in this case very small. Without high chromaticity and octupole current, the halo removal rates are small and a pulsing of the HEL is considered in this case. 
3. Magnetic errors: Magnetic errors in general increase the halo removal rate (see Fig. 13). In this paper only one seed has been simulated. A consistent study with more statistic would need to be performed in order to properly evaluate the impact.

- An optimal cleaning in both planes is achieved for a round proton beam matched to the round electron beam of the HEL. In case of the HL-LHC the coupling is also too small in order to lead to a sufficient mixing of the planes in order to compensate for the unequality between the planes (see Fig. 13).

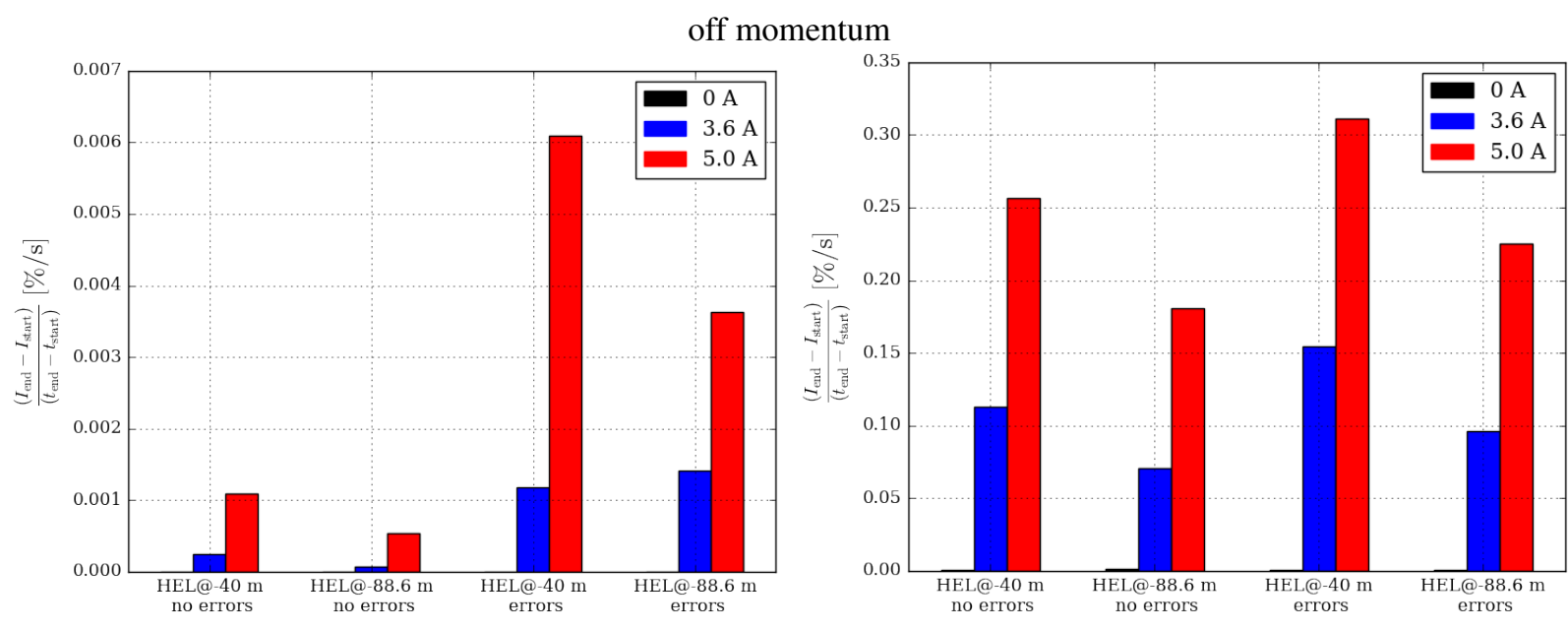

Figure 13. Flat top with and without magnetic errors and HEL installed at $-40 \mathrm{~m}$ and $-88.6 \mathrm{~m}$ : Halo removal rates for the case of a uniform halo distribution between $4-6 \sigma$ transversely and a Gaussian distribution cut at $6 \sigma$ in the longitudinal plane. The removal rates are obtained by averaging the halo losses over $90 \mathrm{~s}$.

- A pulsing of the HEL is particularly interesting at flat top in which case the halo removal rates are small. Two different pulsing modes are considered:

- random: random modulation of the HEL current.

- resonant: the HEL is pulsed every nth turn.

The random pulsing between full and zero current leads to a very strong exponential decay of the intensity (see Fig. 7). For the resonant excitation the losses are rather linear and similar for all different pulsing patterns, but considerably smaller than for the random excitation (Fig. 14). Halo removal rates for the random mode are for on-momentum $0.8 \% / \mathrm{s}$ and off-momentum $0.01 \% / \mathrm{s}-$ $10 \% / \mathrm{s}$. For the resonant excitation removal rates between $0.0004 \% / \mathrm{s}-0.003 \% / \mathrm{s}$ for on-momentum and $0.14 \% / \mathrm{s}-0.22 \% / \mathrm{s}$ for off-momentum are obtained depending on the pulsing pattern.

The resonant pulsing could however be advantageous as one could choose a pulsing pattern affecting the halo but leaving the core untouched. Pulsing patterns not affecting the core in general exist at least for the nominal LHC at injection (to be proven still in the case of HL-LHC). This has been shown 

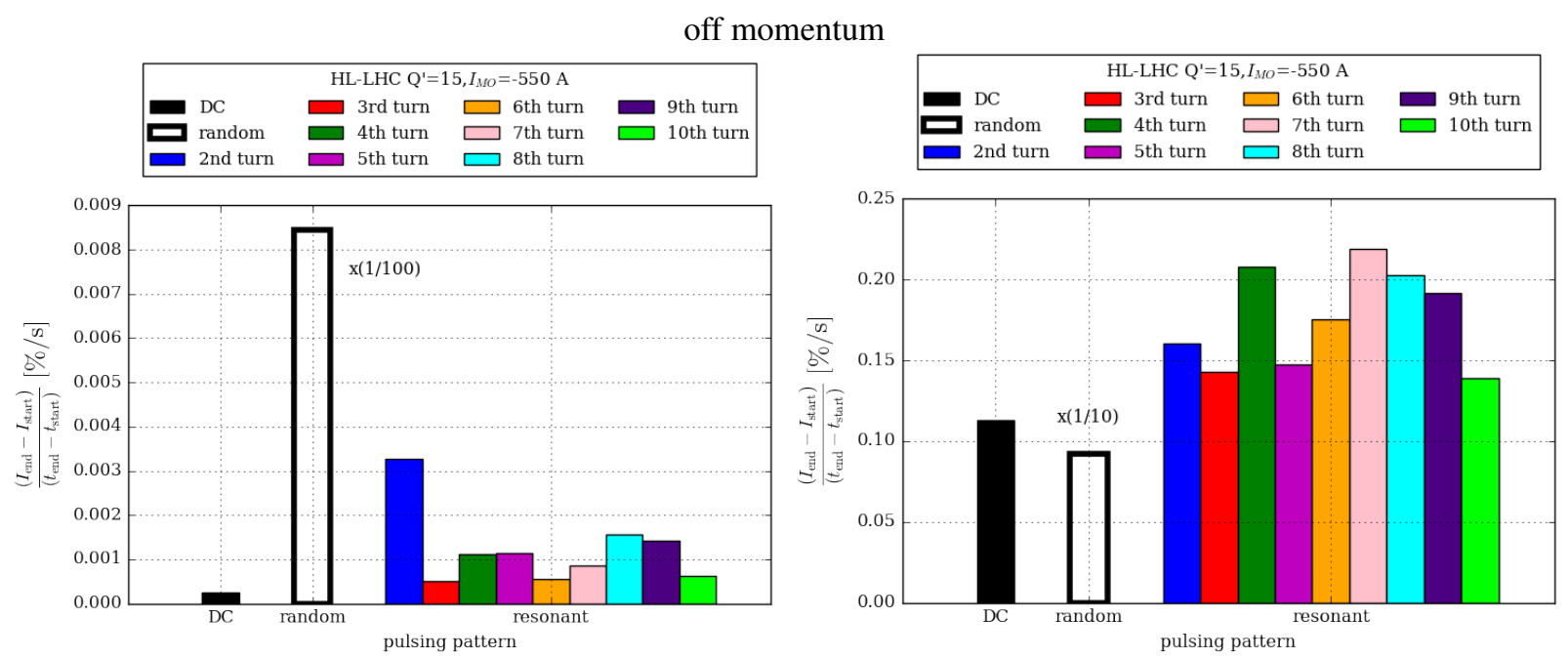

Figure 14. Flat top and pulsed operation of the HEL: Halo removal rates for the case of a uniform halo distribution between $4-6 \sigma$ transversely and a Gaussian distribution cut at $6 \sigma$ in the longitudinal plane. The removal rates are obtained by averaging the halo losses over $90 \mathrm{~s}$.

in simulations using a Gaussian distribution of particles and in experiments at the LHC [6],[10]. In both cases a strong dependence of the losses on the pulsing pattern has been observed.

It has to be also taken into account that in case of a pulsed operation noise is introduced on the core of the proton beam due to the kick generated by the bends of the HEL and profile imperfections in the HEL electron beam (see [6] for further details).

- Two regimes are observed for the uniform transverse distribution between $4-6 \sigma$ and $\frac{\Delta p}{p_{0}}=0$ or Gaussian in $\frac{\Delta p}{p_{0}}$ : an initial exponential decay and a later linear decrease (see Sec. VI). The halo removal rates (or relative intensity) can be thus fitted with 4

$$
I(t)=a+b \cdot t+A \cdot e^{-\frac{t}{\tau}}
$$

The initial exponential decay can be measured with the decay time $\tau$ and the linear continuous decrease with the slope $b$. The halo removal rates for other distributions could be obtained by folding the distribution with the one used in this paper or simply redoing the simulations. The HEL however seems to clean the halo in most cases rather equally in amplitude (Appendix D). In addition also diffusive and damping processes like scattering, intra-beam scattering and synchrotron radiation need to be considered in a realistic model.

\section{ACKNOWLEDGMENTS}

We would like to thank D. Shatilov for his invaluable support with and extension of LifeTrac [9], S. Fartoukh, R. De Maria, R. Tomás and Y. Papaphilippou for their help with the preparation of the lattice and 
optics input file for SixTrack and R. Bruce and S. Redaelli for their advice. 


\section{Appendix A: DA simulations for luminosity leveling from $0.7 \mathrm{~m}$ to $0.15 \mathrm{~m}$.}

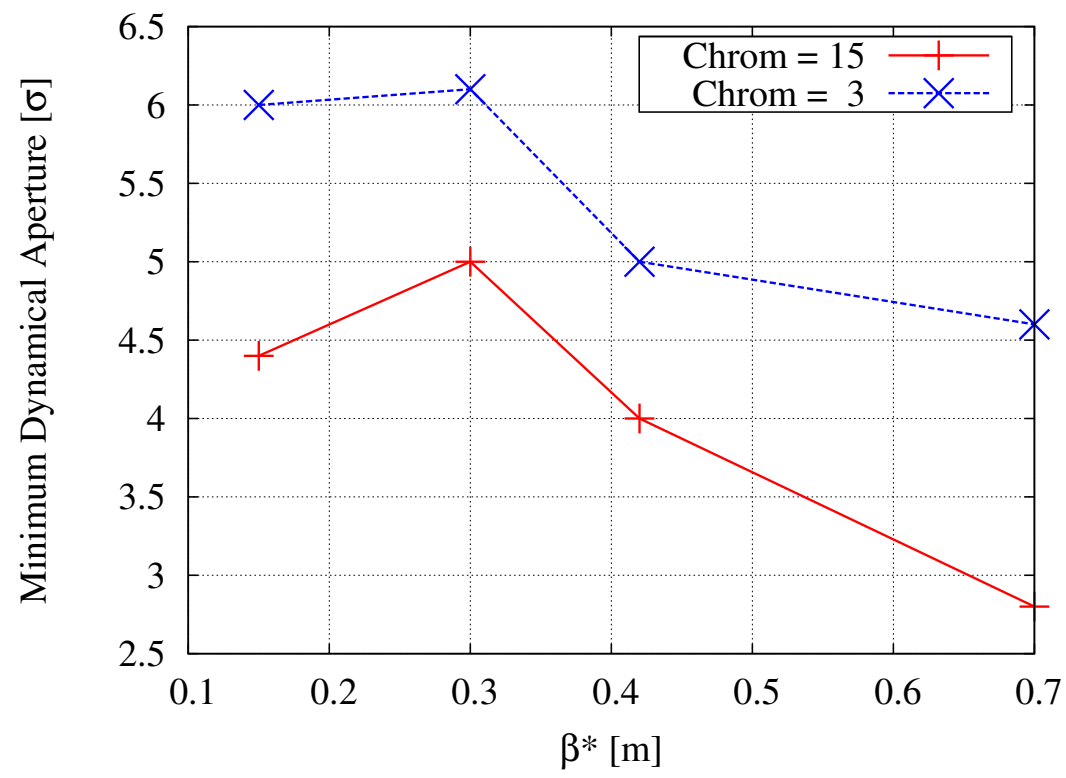

Figure 15. Minimum dynamic aperture determined with LifeTrac for the $\beta^{*}$ leveling scenario described in Table ?? and two different values of chromaticity. The spectrometer polarity in IP2/8 yielding the smallest DA has been chosen. For beam-beam full crabbing is assumed in IP1/5. The layout version V1.0 has been used, the simulations do not include any errors and the current of the focusing octupoles (MOF) is set to $I_{\mathrm{MO}}=-550$. 


\section{Appendix B: Comparison of fit parameters and average halo removal rates}

\section{Fit parameters and average halo removal rate at flat top}

a. Fit parameters and average halo removal rate: flat top and different chromaticity and octupole settings for HEL installed at $-40 \mathrm{~m}$
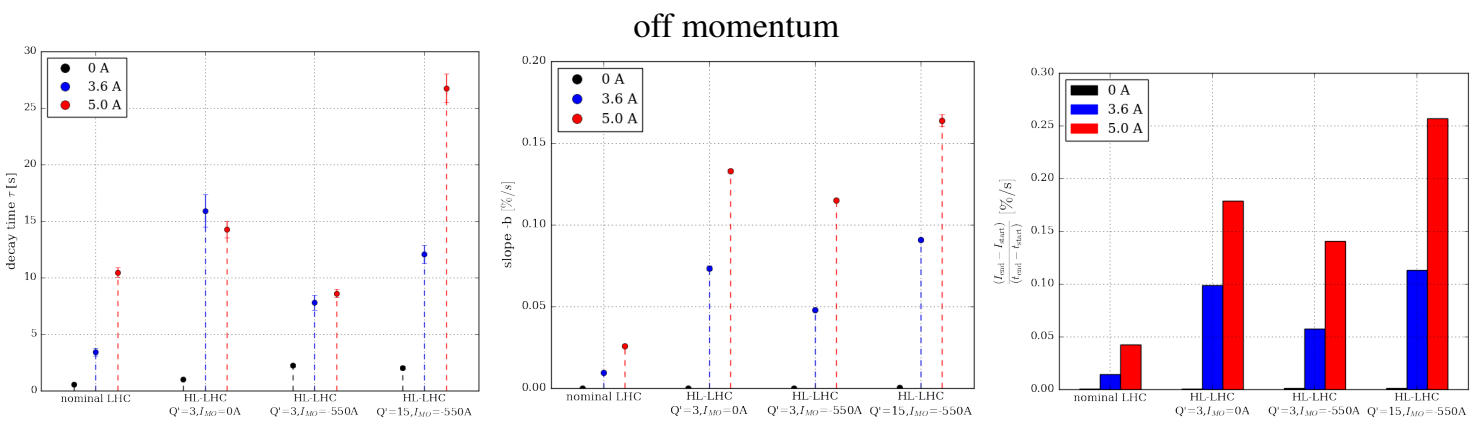

Figure 16. Flat top and different chromaticity and octupole settings: Decay time $\tau$, slope $b$ (center)and average halo removal rate (right) obtained from fit with function defined in Eqn 4. Only the results for a off-momentum (Gaussian distribution in the longitudinal) is shown as for on-momentum $\left(\left(z, \frac{\Delta p}{p_{0}}\right)=(0,0)\right)$ the losses are too small in order to obtain a proper fit. The errorbars indicate the $1 \sigma$ standard deviation obtained from the covariance matrix of the fit. 
b. Fit parameters and average halo removal rate: flat top with magnet errors and HEL at $-40 \mathrm{~m}$ and $-88.6 \mathrm{~m}$ :

$$
Q^{\prime}=15, I_{\mathrm{MO}}=-550 \mathrm{~A}
$$
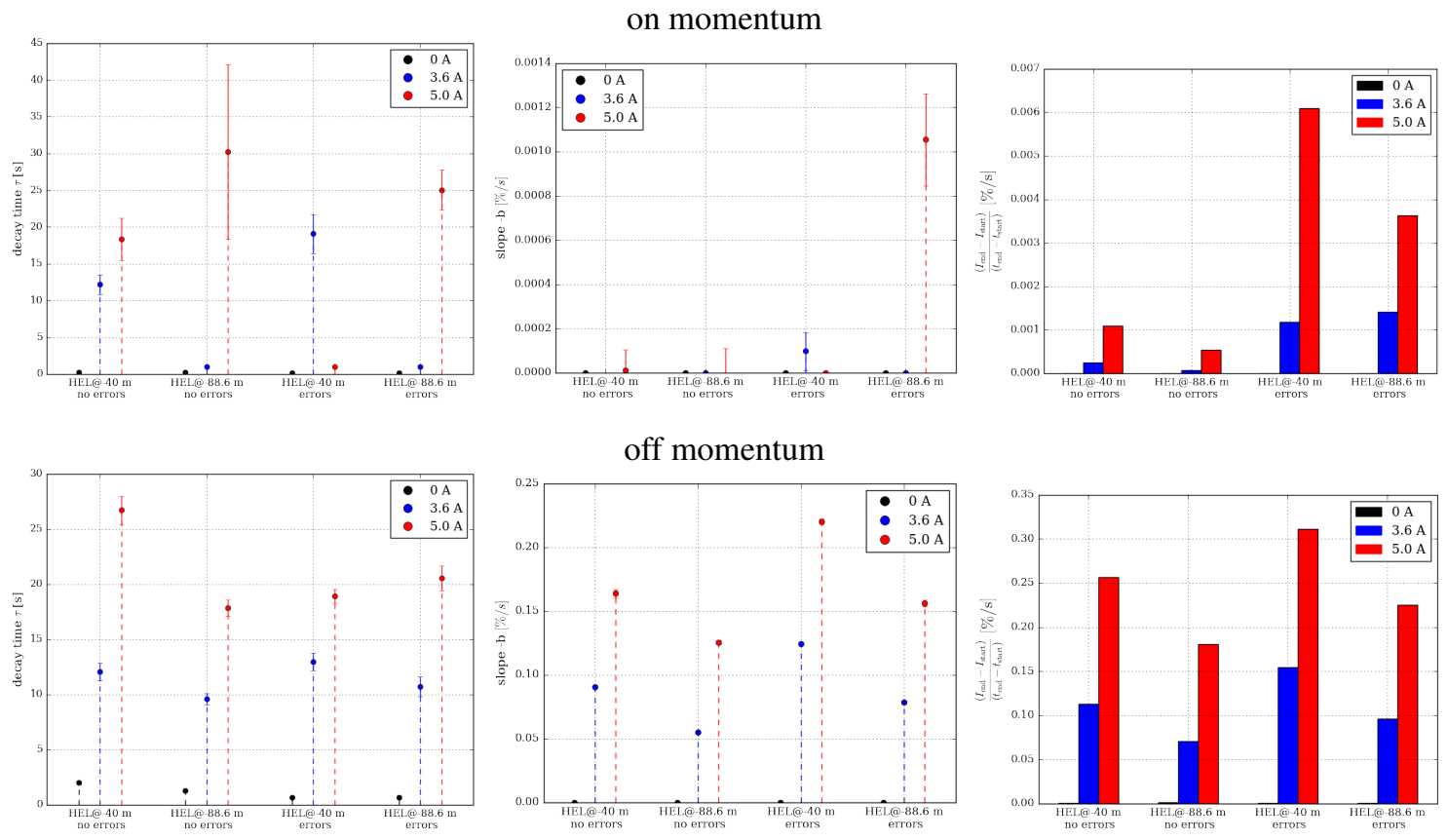

Figure 17. Flat top with and without magnetic errors and HEL installed at $-40 \mathrm{~m}$ and $-88.6 \mathrm{~m}$ : Decay time $\tau$, slope $b$ (center)and average halo removal rate (right) obtained from fit with function defined in Eqn 4. The errorbars indicate the $1 \sigma$ standard deviation obtained from the covariance matrix of the fit. 
c. Fit parameters and average halo removal rate: flat top with pulsing: $Q^{\prime}=15, I_{\mathrm{MO}}=-550 \mathrm{~A}$
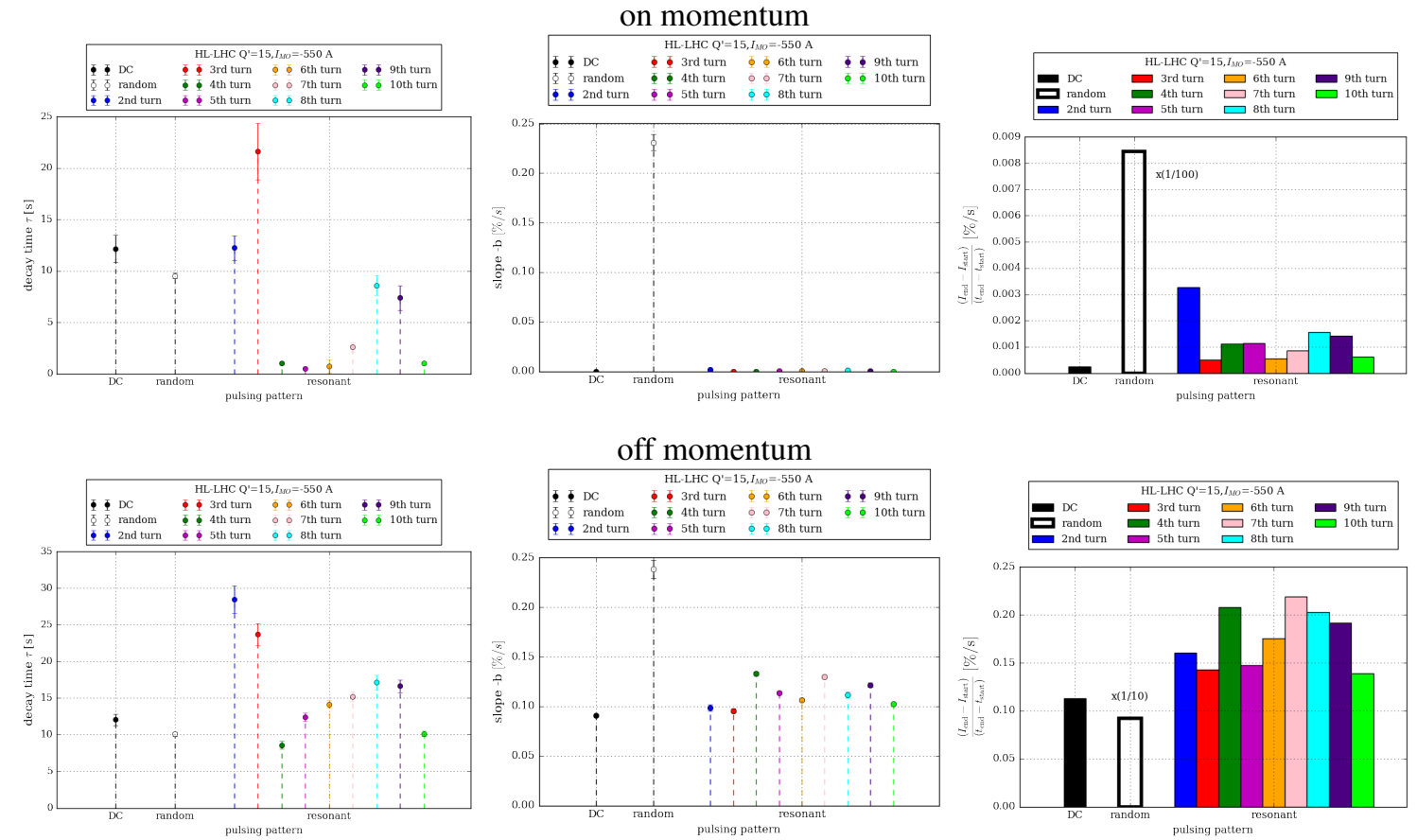

Figure 18. Flat top with and without magnetic errors and HEL installed at $-40 \mathrm{~m}$ and $-88.6 \mathrm{~m}$ :Decay time $\tau$, slope $b$ (center)and average halo removal rate (right) obtained from fit with function defined in Eqn 4. The errorbars indicate the $1 \sigma$ standard deviation obtained from the covariance matrix of the fit. 
2. Fit parameters: $\beta^{*}$-leveling cases for HEL installed at $-\mathbf{4 0} \mathrm{m}$
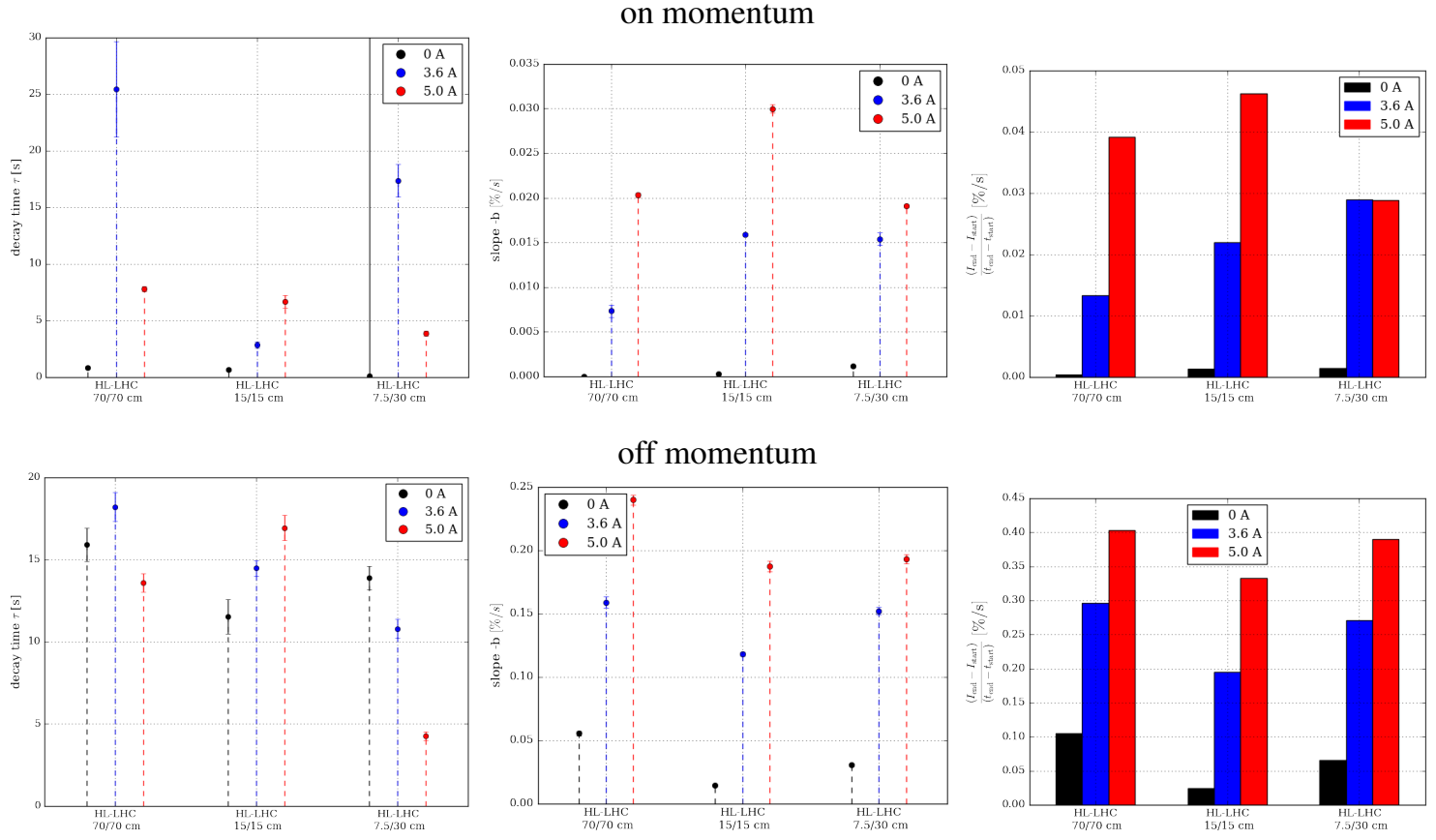

Figure 19. $\beta^{*}$ leveling: Decay time $\tau$, slope $b$ (center)and average halo removal rate (right) obtained from fit with function defined in Eqn 4. The errorbars indicate the $1 \sigma$ standard deviation obtained from the covariance matrix of the fit. 


\section{Appendix C: FMA analysis}

\section{FMA analysis for flat top}

a. FMA analysis: flat top and different chromaticity and octupole settings for HEL installed at $-40 \mathrm{~m}$

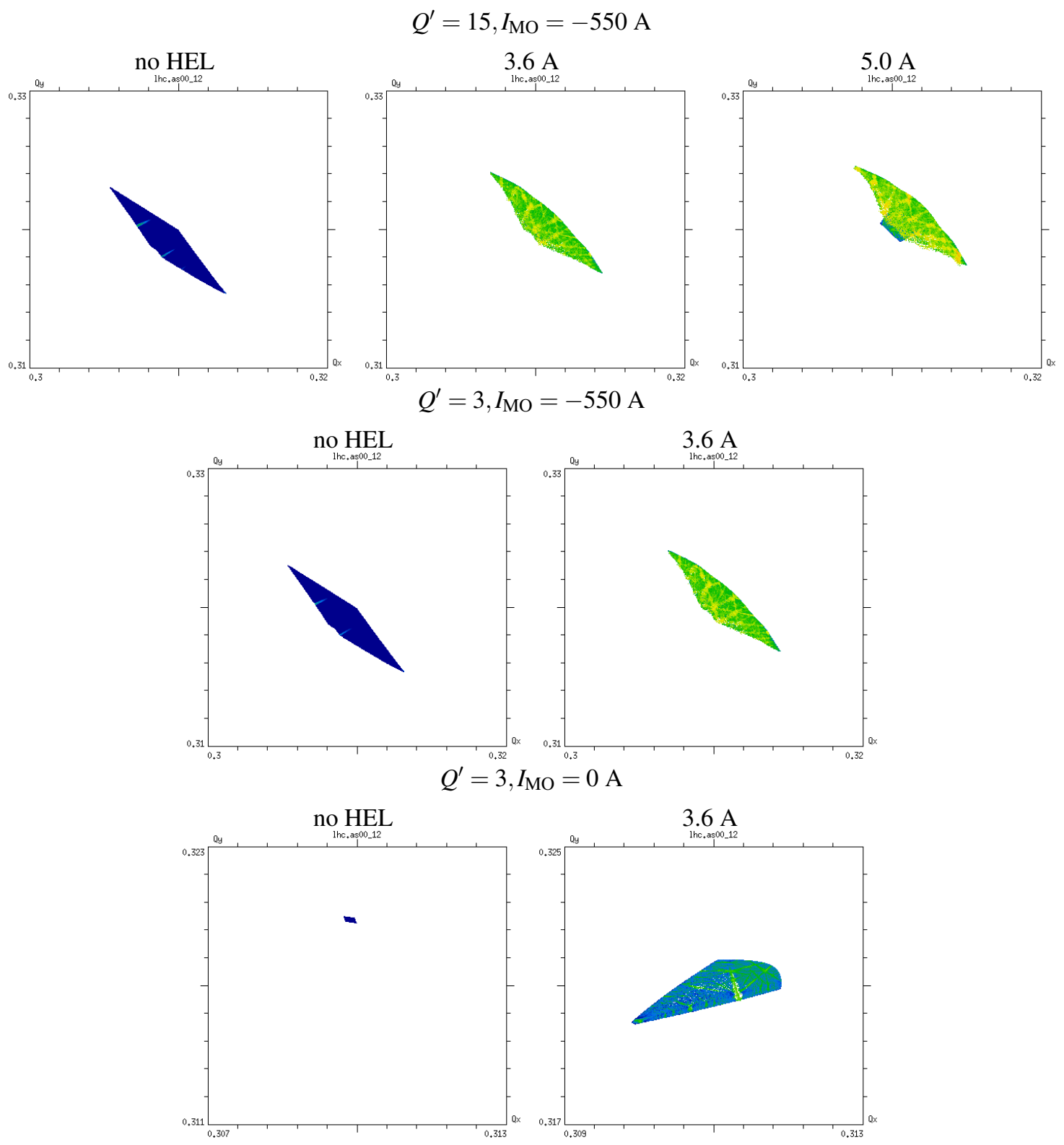

Figure 20. flat top, HEL installed at $-40 \mathrm{~m}$ : FMA analysis for on-momentum particles $\left(\frac{\Delta p}{p_{0}}=0.0 \sigma_{p}\right)$ up to $10 \sigma$ amplitude for a quadratric grid with and without HEL. 


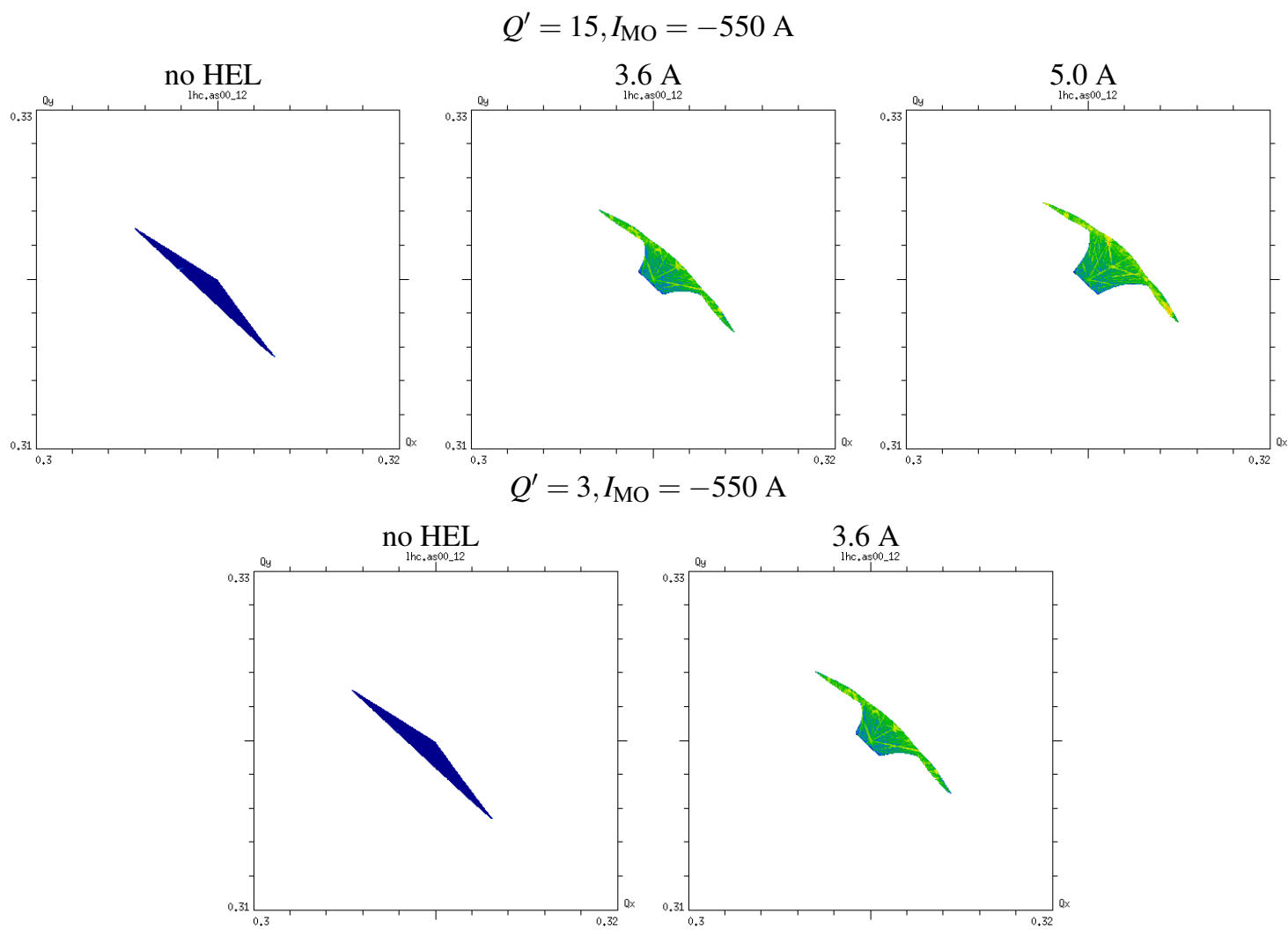

Figure 21. flat top, HEL installed at $-40 \mathrm{~m}$ : FMA analysis for on-momentum particles $\left(\frac{\Delta p}{p_{0}}=0.0 \sigma_{p}\right)$ up to $10 \sigma$ amplitude for a circular grid with and without HEL. 


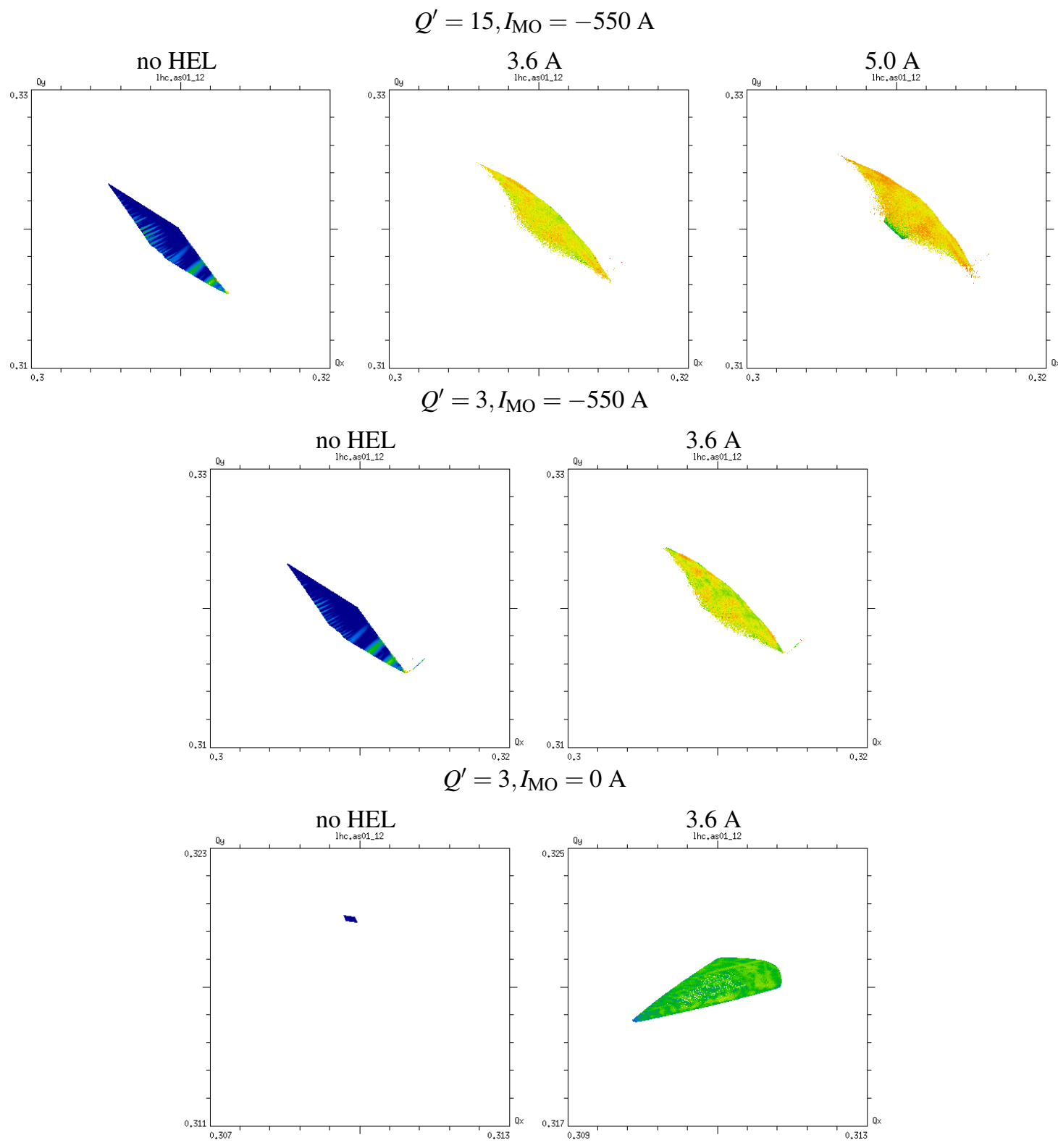

Figure 22. flat top, HEL installed at $-40 \mathrm{~m}$ : FMA analysis for off-momentum particles ( $\left.\frac{\Delta p}{p_{0}}=0.1\right)$ up to $10 \sigma$ amplitude for a quadratric grid with and without HEL. 


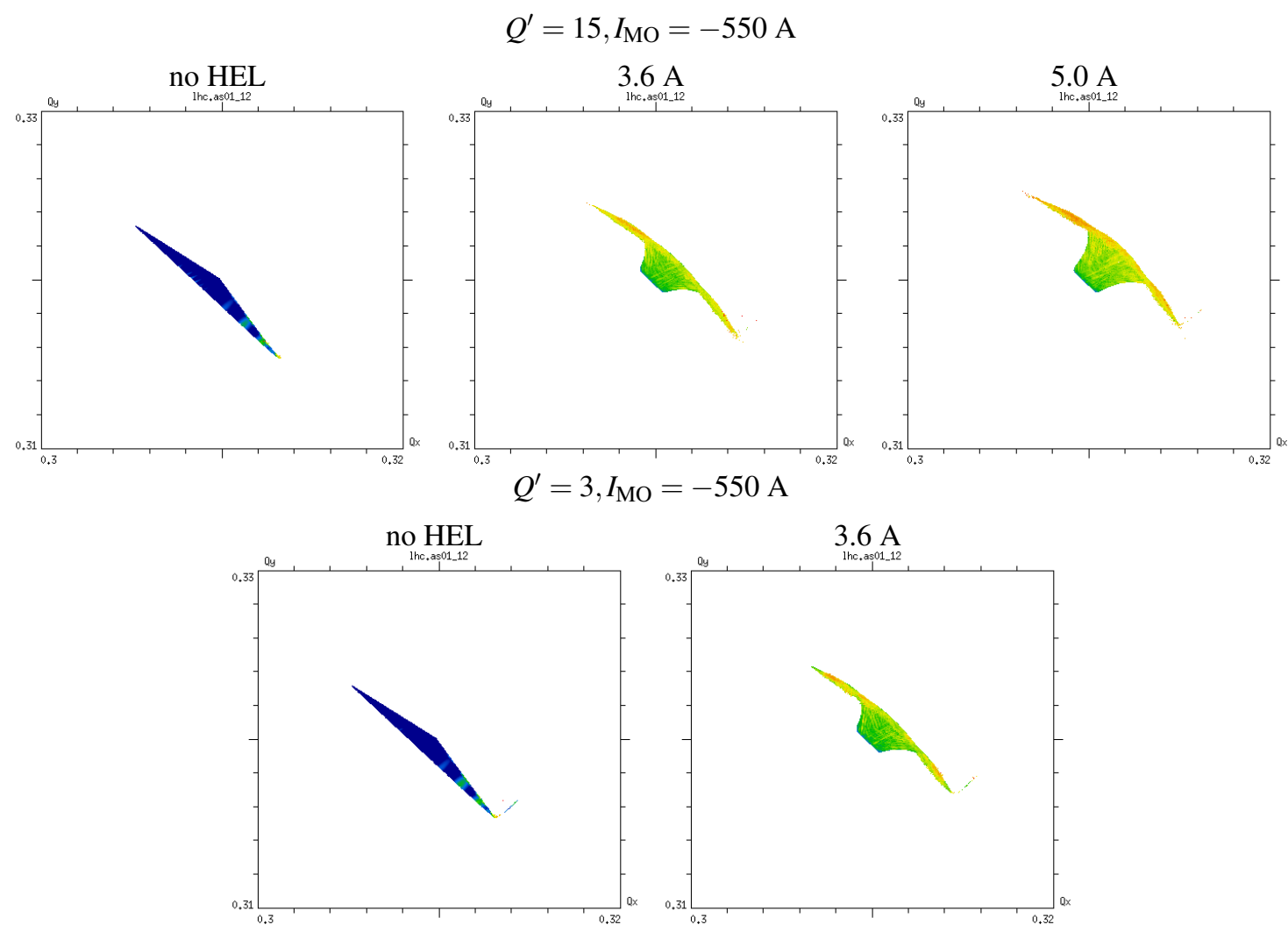

Figure 23. flat top, HEL installed at -40 m: FMA analysis for off-momentum particles ( $\left.\frac{\Delta p}{p_{0}}=0.1\right)$ up to $10 \sigma$ amplitude for a circular grid with and without HEL. 

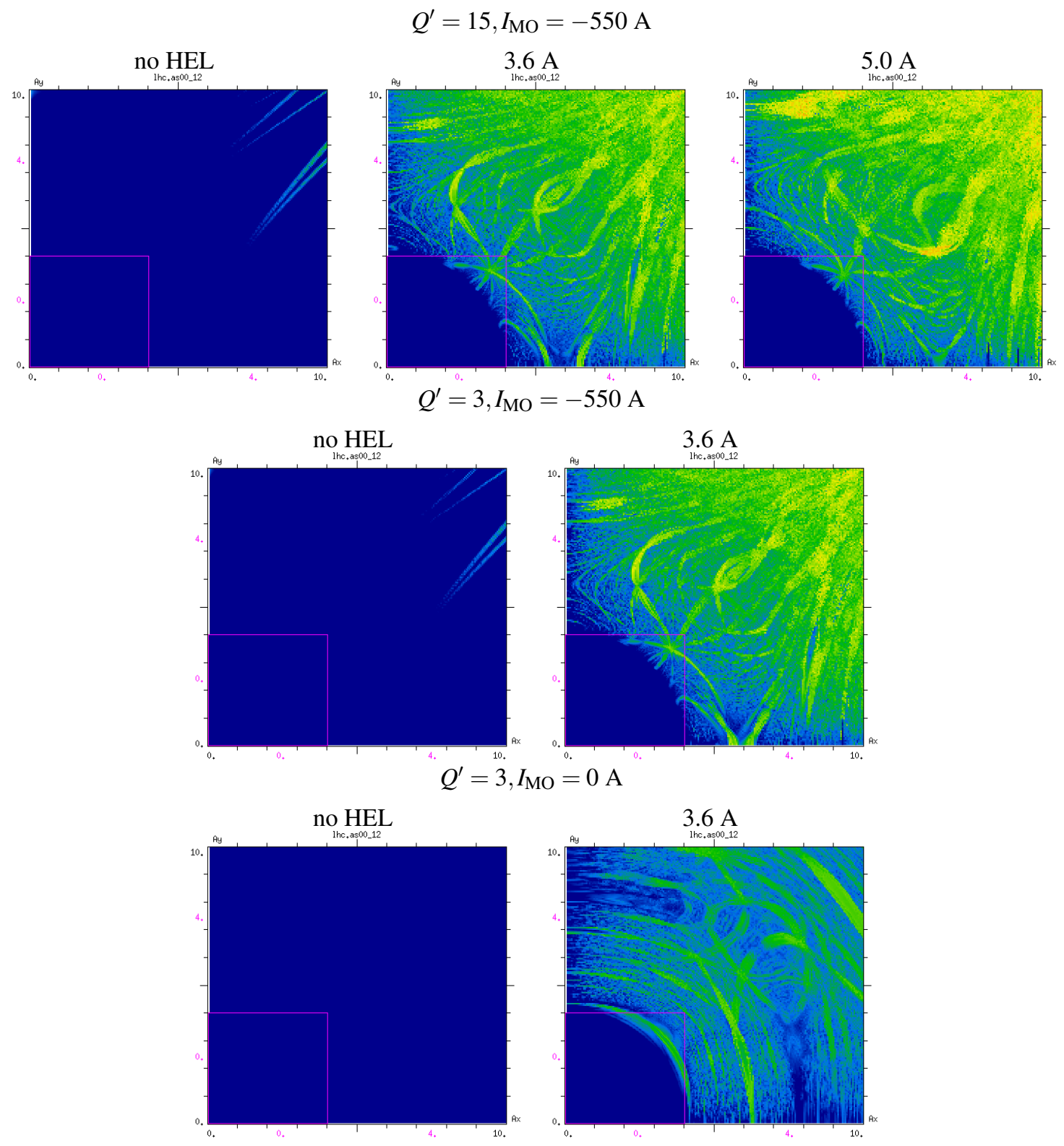

Figure 24. flat top, HEL installed at -40 m: FMA analysis in amplitude space for on-momentum particles $\left(\frac{\Delta p}{p_{0}}=0\right)$ up to $10 \sigma$ amplitude for a quadratric grid with and without HEL. 

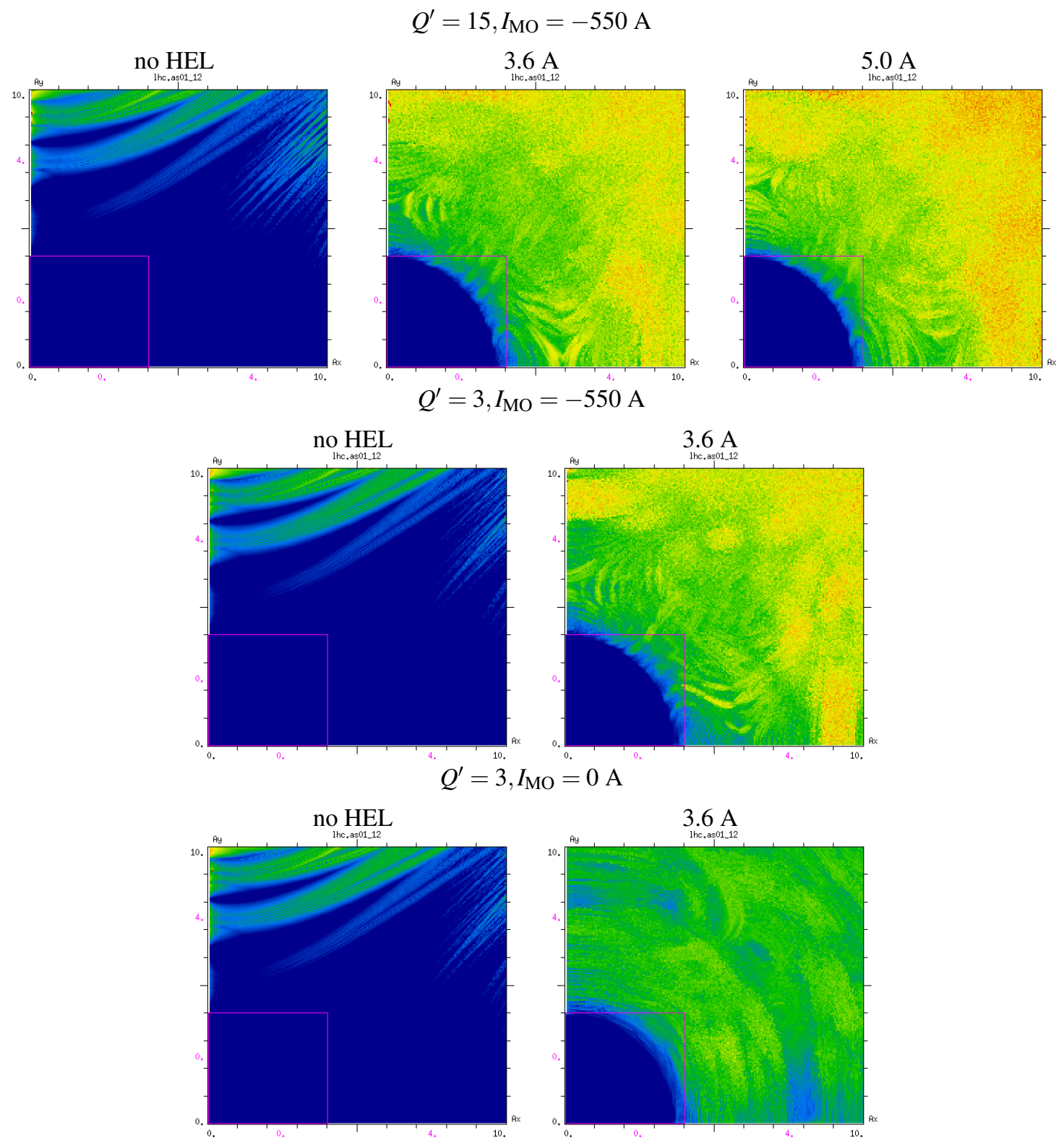

Figure 25. flat top, $Q^{\prime}=15, I_{\mathrm{MO}}=-550 \mathrm{~A}$, HEL installed at $-40 \mathrm{~m}$ : FMA analysis in amplitude space for offmomentum particles $\left(\frac{\Delta p}{p_{0}}=0.1 \sigma_{p}\right)$ up to $10 \sigma$ amplitude for a quadratric grid with and without HEL. 
b. FMA analysis: flat top with magnet errors and HEL at $-40 \mathrm{~m}$ and $-88.6 \mathrm{~m}: Q^{\prime}=15, I_{\mathrm{MO}}=-550 \mathrm{~A}$
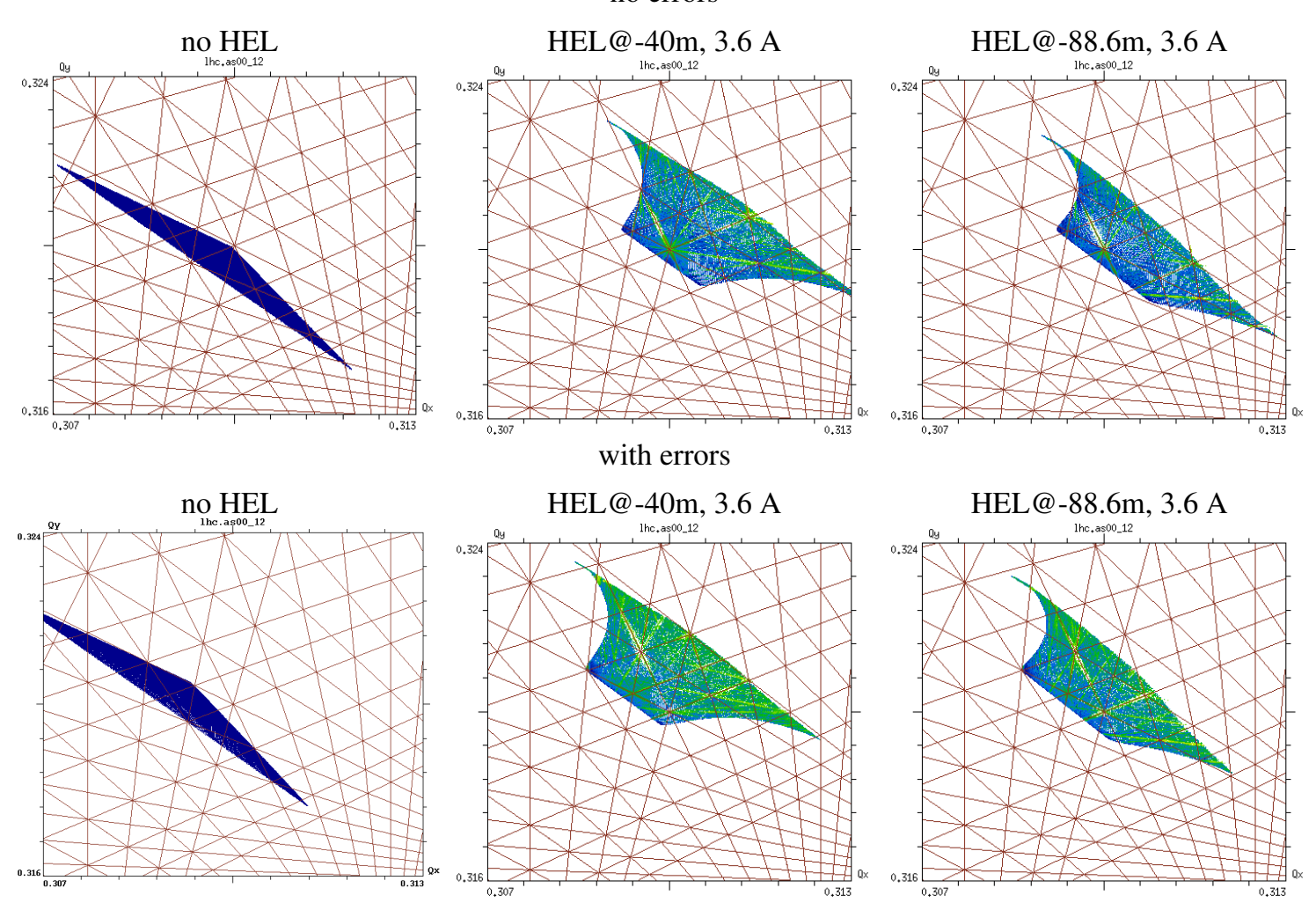

Figure 26. flat top with (bottom) and without (top) magnetic errors, $Q^{\prime}=15, I_{\mathrm{MO}}=-550 \mathrm{~A}$, HEL installed at $-40 \mathrm{~m}$ and -88.6 m: FMA analysis for on-momentum particles $\left(\frac{\Delta p}{p_{0}}=0.0 \sigma_{p}\right)$ up to $8 \sigma$ amplitude for a circular grid with and without HEL. 

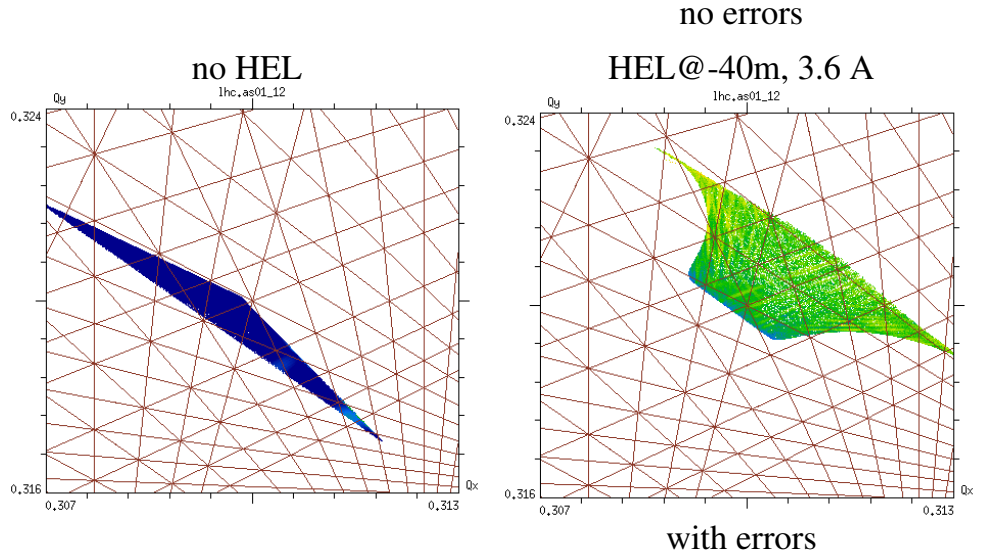

no errors
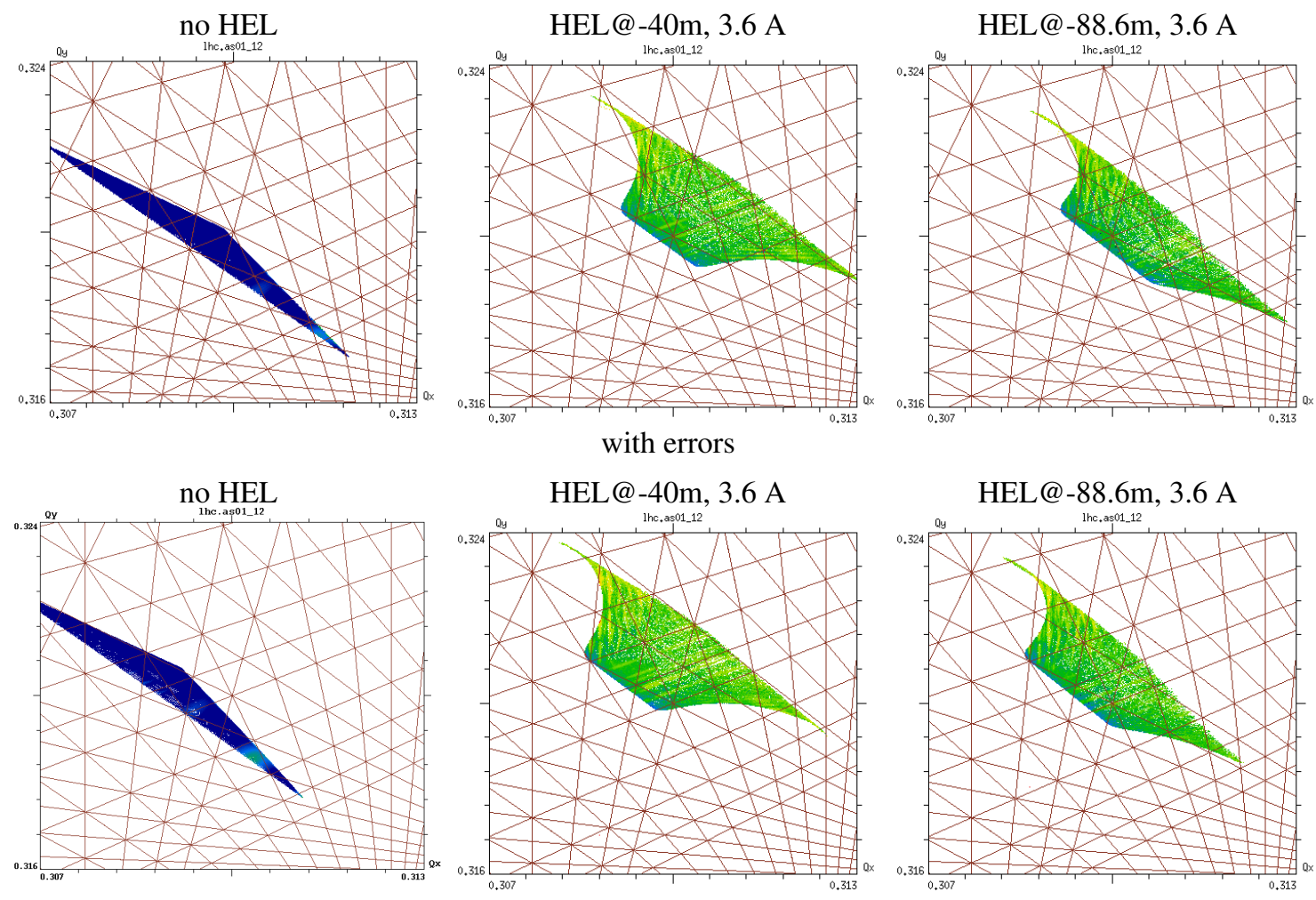

Figure 27. flat top with (bottom) and without (top) magnetic errors, $Q^{\prime}=15, I_{\mathrm{MO}}=-550 \mathrm{~A}$, HEL installed at $-40 \mathrm{~m}$ and -88.6 m: FMA analysis for off-momentum particles $\left(\frac{\Delta p}{p_{0}}=0.1 \sigma_{p}\right)$ up to $8 \sigma$ amplitude for a circular grid with and without HEL. 


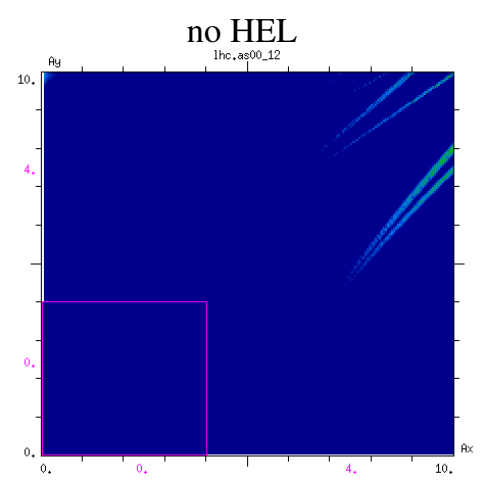

no HEL

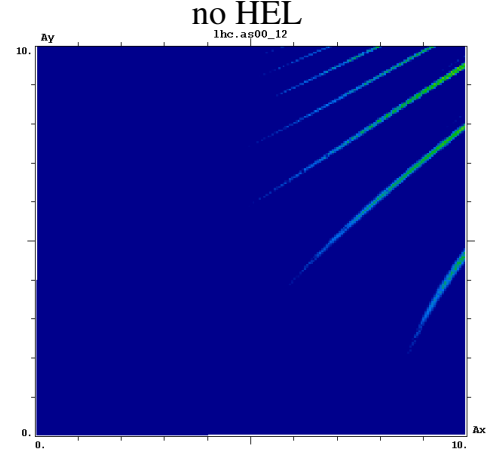

no errors

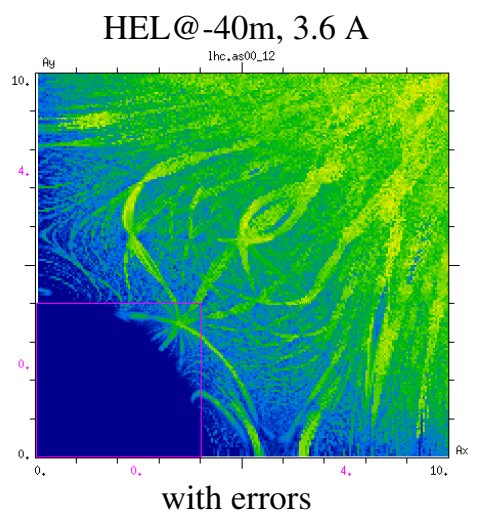

HEL@-40m, 3.6 A

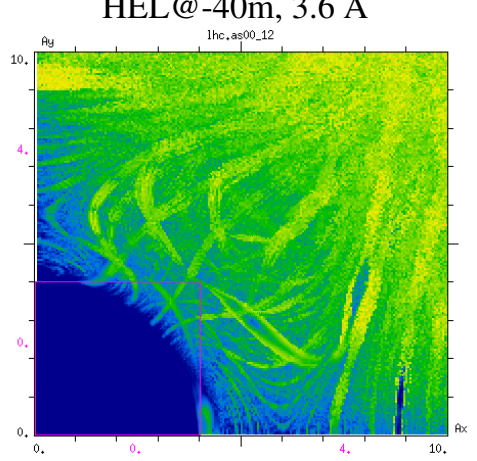

HEL@-88.6m, 3.6 A

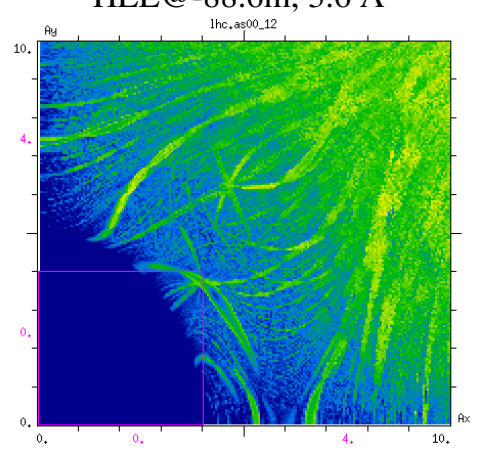

HEL@-88.6m, 3.6 A

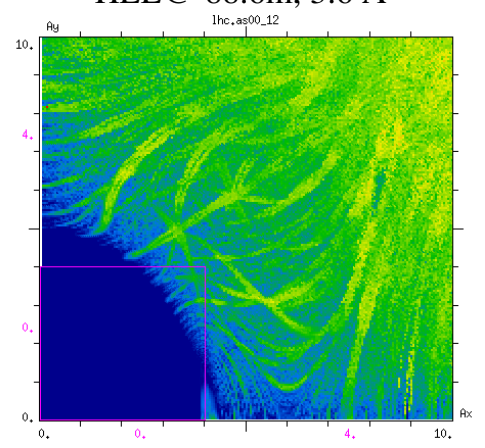

Figure 28. flat top with (bottom) and without (top) magnetic errors, $Q^{\prime}=15, I_{\mathrm{MO}}=-550 \mathrm{~A}$, HEL installed at $-40 \mathrm{~m}$ and -88.6 m: FMA analysis in amplitude space for on-momentum particles $\left(\frac{\Delta p}{p_{0}}=0.0 \sigma_{p}\right)$ up to $10 \sigma$ amplitude for a rectangular grid with and without HEL. 

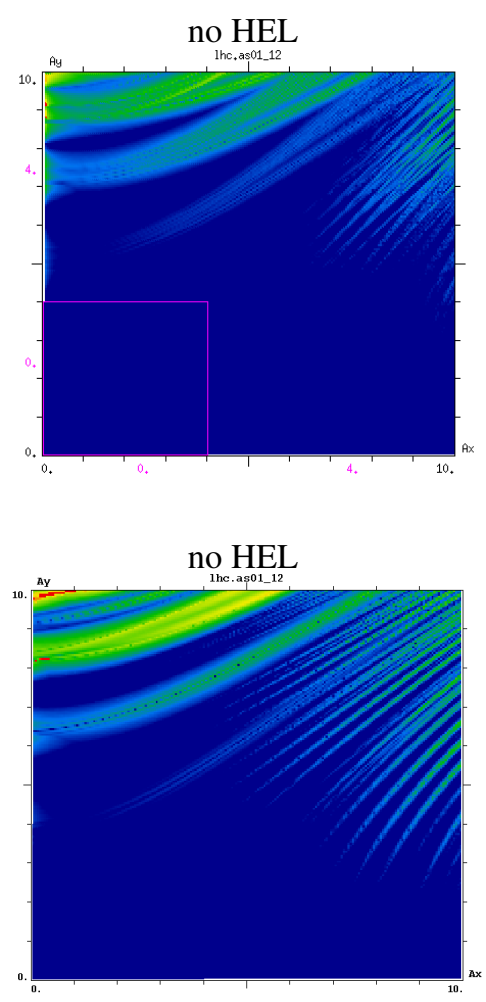

no errors

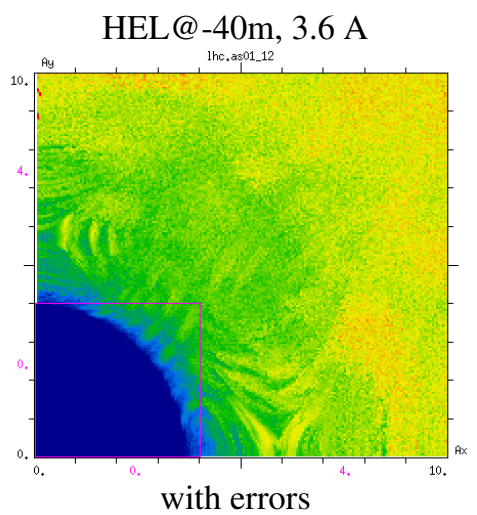

HEL@-40m, 3.6 A

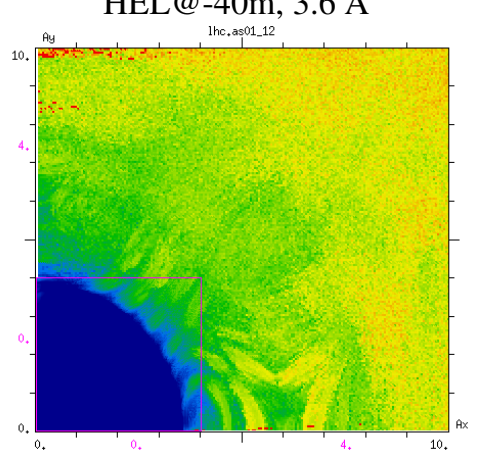

HEL@-88.6m, 3.6 A

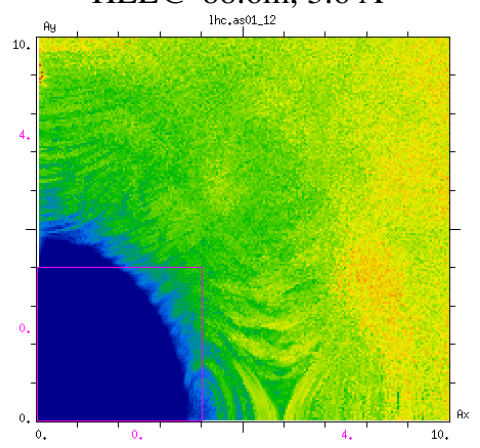

HEL@-88.6m, 3.6 A

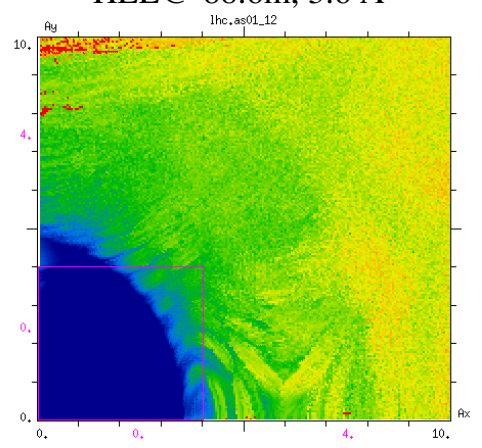

Figure 29. flat top with (bottom) and without (top) magnetic errors, $Q^{\prime}=15, I_{\mathrm{MO}}=-550 \mathrm{~A}$, HEL installed at $-40 \mathrm{~m}$ and -88.6 m: FMA analysis in amplitude space for off-momentum particles $\left(\frac{\Delta p}{p_{0}}=0.1 \sigma_{p}\right)$ up to $10 \sigma$ amplitude for a rectangular grid with and without HEL. 
c. FMA analysis: flat top with pulsing and $Q^{\prime}=15, I_{\mathrm{MO}}=-550 \mathrm{~A}$
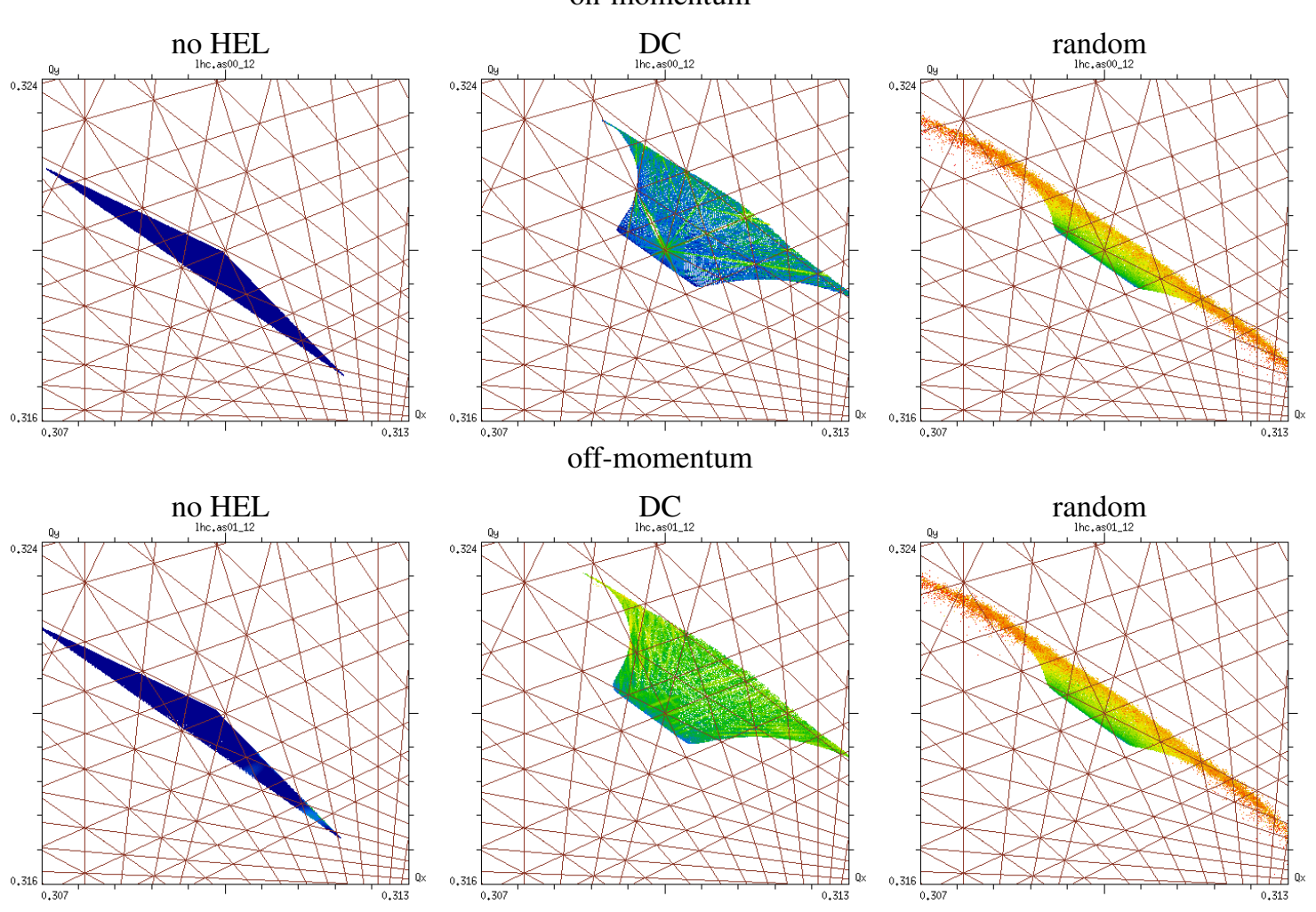

Figure 30. flat top, $Q^{\prime}=15, I_{\mathrm{MO}}=-550 \mathrm{~A}$, HEL installed at $-40 \mathrm{~m}$, random pulsing: FMA analysis for on-momentum $\left(\frac{\Delta p}{p_{0}}=0.0 \sigma_{p}\right)$ (top) and off-momentum particles $\left(\frac{\Delta p}{p_{0}}=0.1 \sigma_{p}\right)$ (bottom) up to $8 \sigma$ amplitude for a circular grid and different pulsing patterns. The resonance lines up to 20th order are indicated in red. 


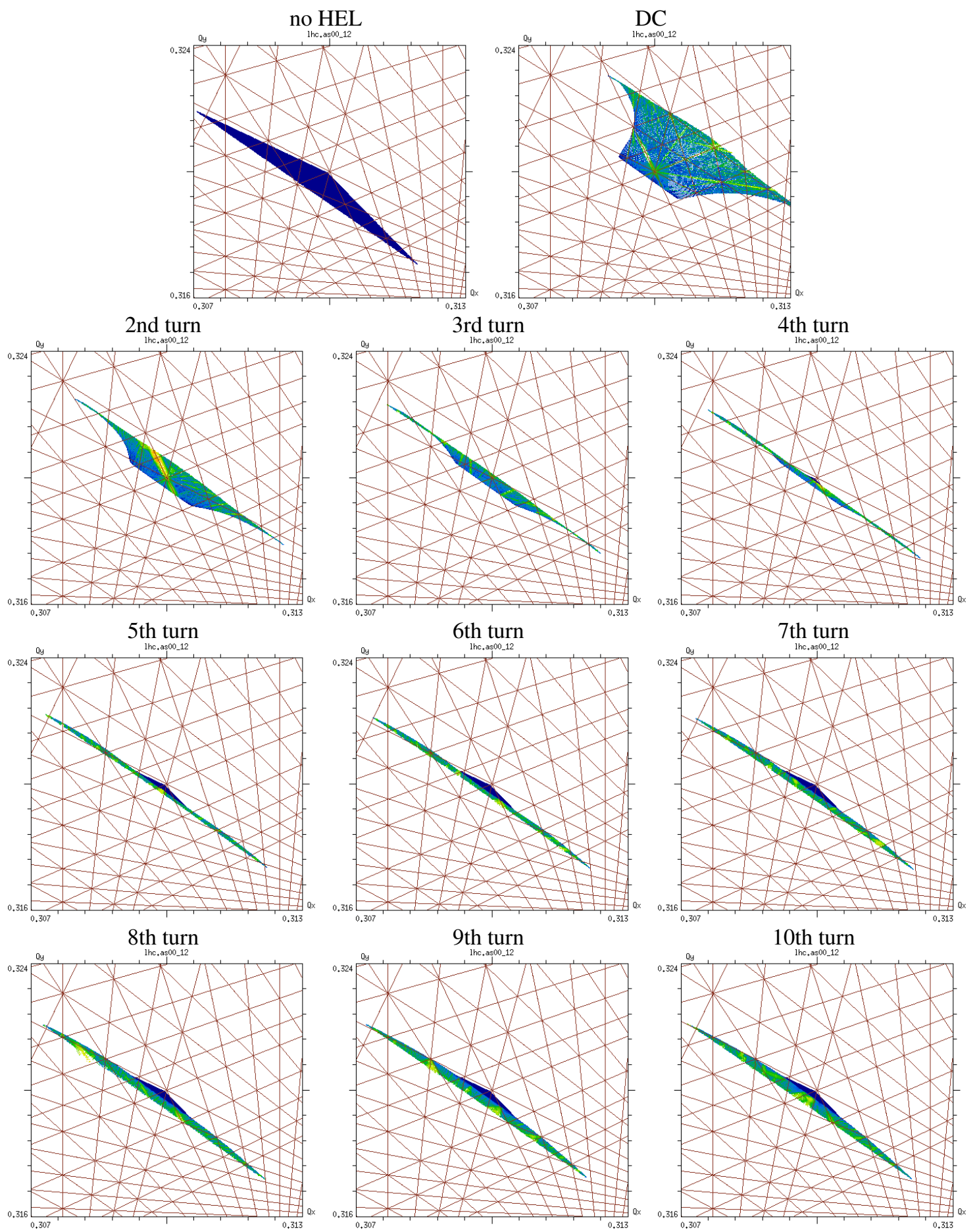

Figure 31. flat top, $Q^{\prime}=15, I_{\mathrm{MO}}=-550 \mathrm{~A}$, HEL installed at $-40 \mathrm{~m}$, resonant pulsing: FMA analysis for on-momentum particles $\left(\frac{\Delta p}{p_{0}}=0.0 \sigma_{p}\right)$ up to $8 \sigma$ amplitude for a circular grid and different pulsing patterns. The resonance lines up to 20 th order are indicated in red. 


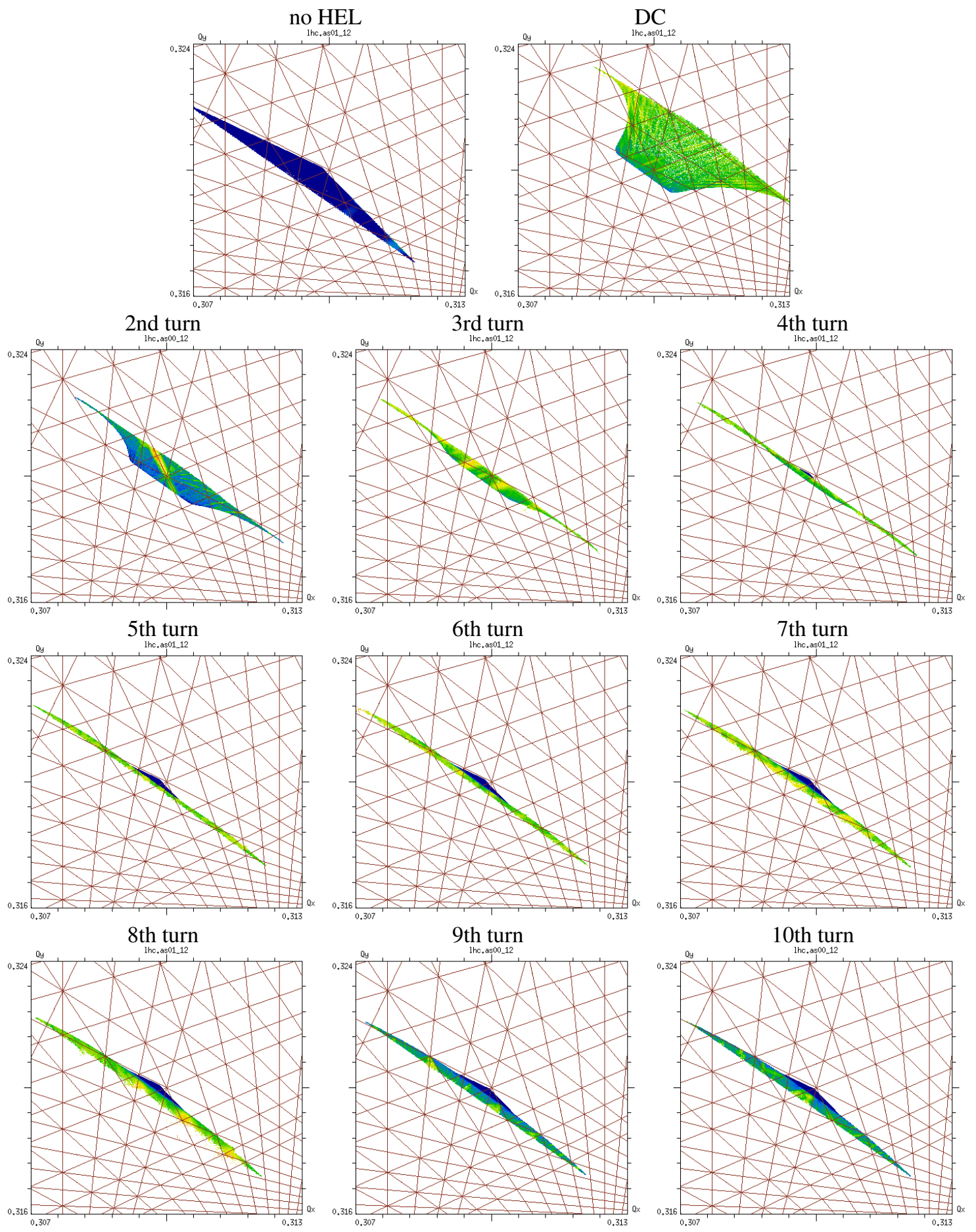

Figure 32. flat top, $Q^{\prime}=15, I_{\mathrm{MO}}=-550 \mathrm{~A}$, HEL installed at $-40 \mathrm{~m}$, resonant pulsing: FMA analysis for offmomentum particles $\left(\frac{\Delta p}{p_{0}}=0.1\right)$ up to $8 \sigma$ amplitude for a circular grid and different pulsing patterns. The resonance lines up to 20th order are indicated in red. 


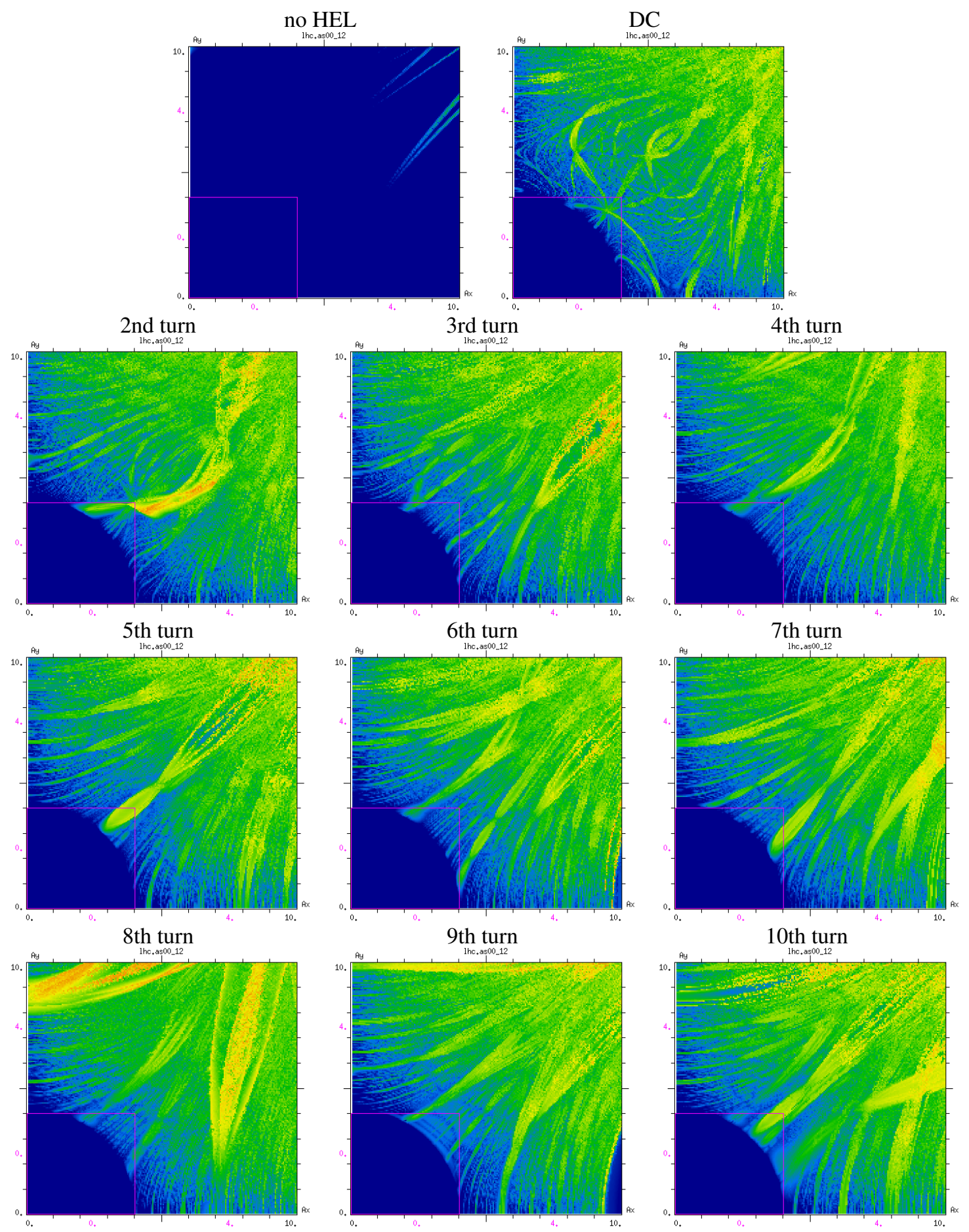

Figure 33. flat top, $Q^{\prime}=15, I_{\mathrm{MO}}=-550 \mathrm{~A}$, HEL installed at $-40 \mathrm{~m}$, resonant pulsing: FMA analysis in amplitude space for on-momentum particles $\left(\frac{\Delta p}{p_{0}}=0.0 \sigma_{p}\right)$ up to $10 \sigma$ amplitude for a rectangular grid and different pulsing patterns. 


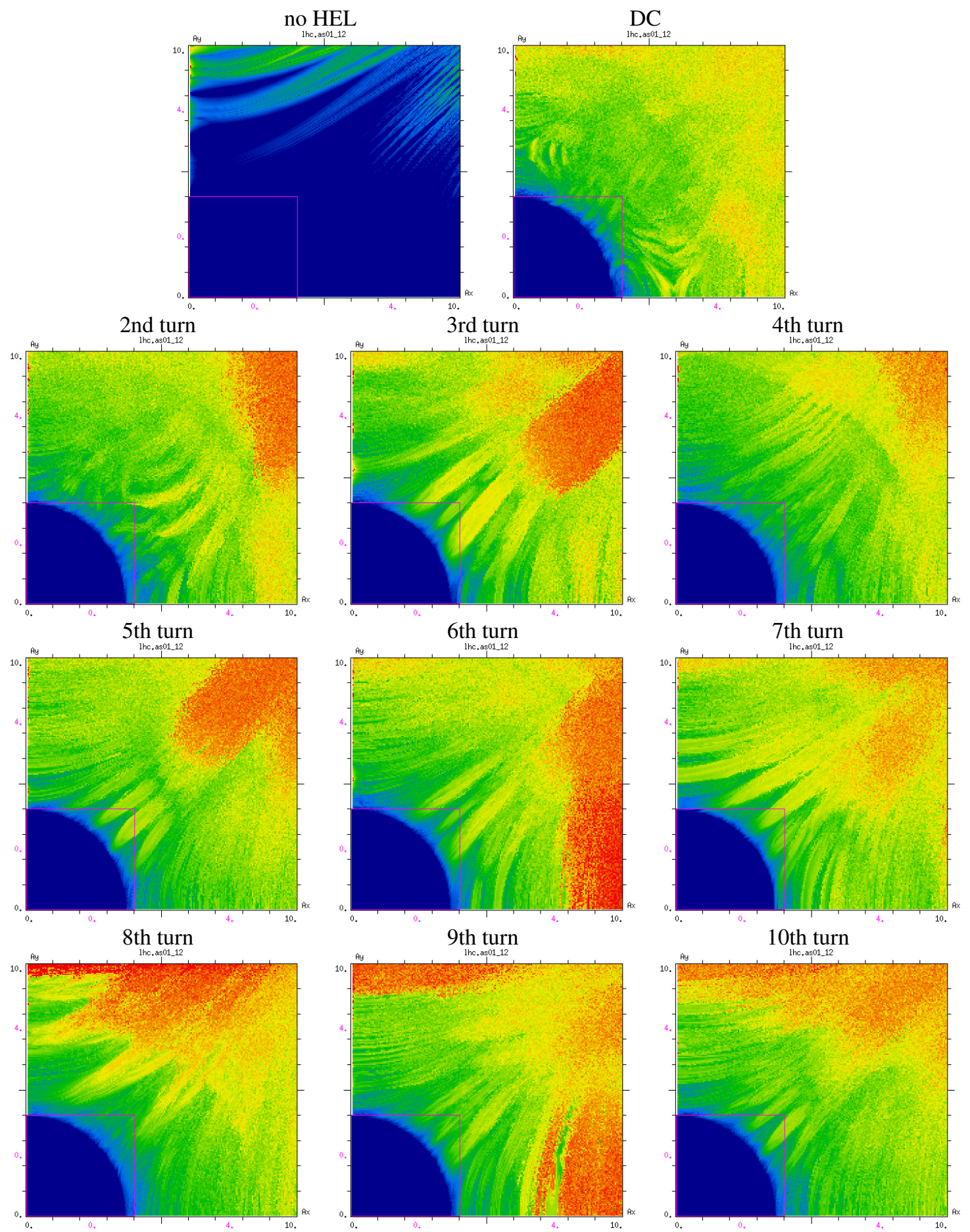

Figure 34. flat top, $Q^{\prime}=15, I_{\mathrm{MO}}=-550 \mathrm{~A}$, HEL installed at $-40 \mathrm{~m}$, resonant pulsing: FMA analysis in amplitude space for off-momentum particles $\left(\frac{\Delta p}{p_{0}}=0.1 \sigma_{p}\right)$ up to $10 \sigma$ amplitude for a rectangular grid and different pulsing patterns. 
2. FMA analysis: $\beta^{*}$-leveling cases for HEL installed at $\mathbf{- 4 0} \mathbf{~ m}$ 
$\beta_{x / y}^{*}=70 / 70 \mathrm{~cm}$ quadratic grid
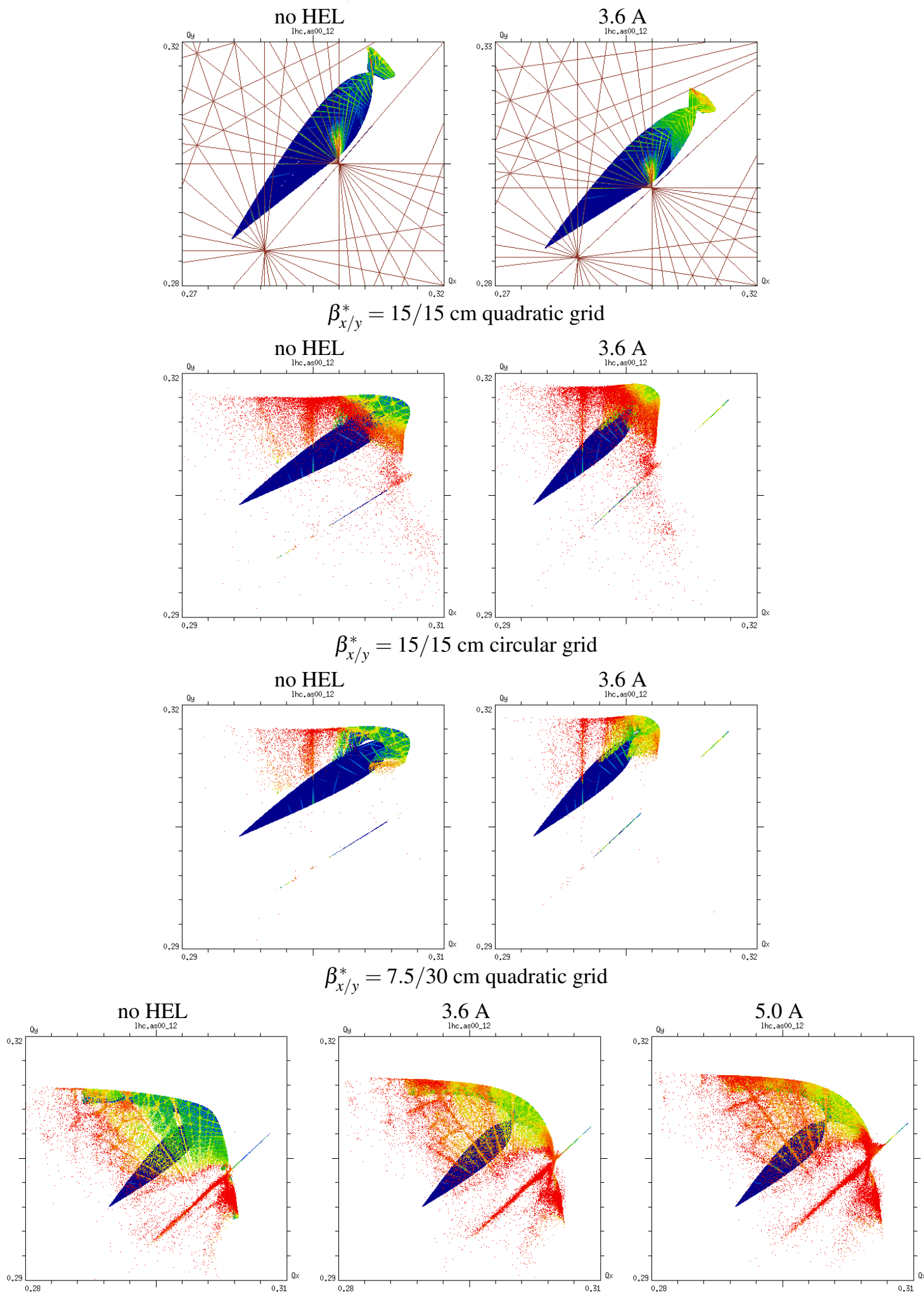

Figure 35. $\beta^{*}$-leveling, $Q^{\prime}=3, I_{\mathrm{MO}}=-550 \mathrm{~A}$, HEL installed at $-40 \mathrm{~m}$ : FMA analysis for on-momentum particles $\left(\frac{\Delta p}{p_{0}}=0.0 \sigma_{p}\right)$ up to $10 \sigma$ amplitude for a quadratric grid with and without HEL. For $\beta_{x / y}^{*}=15 / 15 \mathrm{~cm}$ also the circular grid is shown in order to reveal the resonances for smaller amplitudes otherwise covered by the backfolded tune footprint. The resonance lines up to 10 th order are indicated in red for $\beta_{x / y}^{*}=70 / 70 \mathrm{~cm}$. 

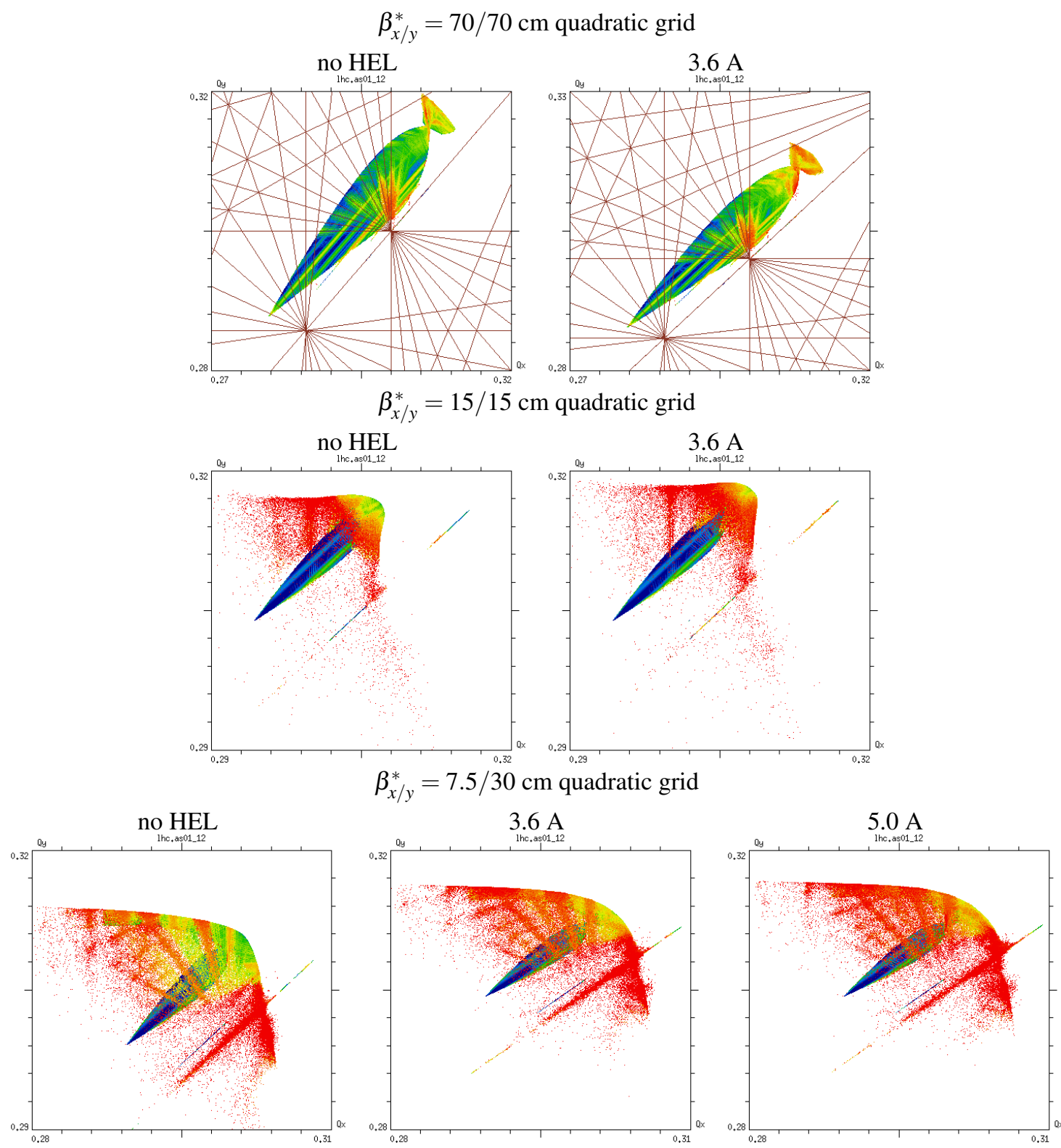

Figure 36. $\beta^{*}$-leveling, $Q^{\prime}=3, I_{\mathrm{MO}}=-550 \mathrm{~A}$, HEL installed at $-40 \mathrm{~m}$ : FMA analysis for off-momentum particles $\left(\frac{\Delta p}{p_{0}}=0.1\right)$ up to $10 \sigma$ amplitude for a quadratric grid with and without HEL. For $\beta_{x / y}^{*}=15 / 15 \mathrm{~cm}$ also the circular grid is shown in order to reveal the resonances for smaller amplitudes otherwise covered by the backfolded tune footprint. The resonance lines up to $10 \mathrm{th}$ order are indicated in red for $\beta_{x / y}^{*}=70 / 70 \mathrm{~cm}$. 

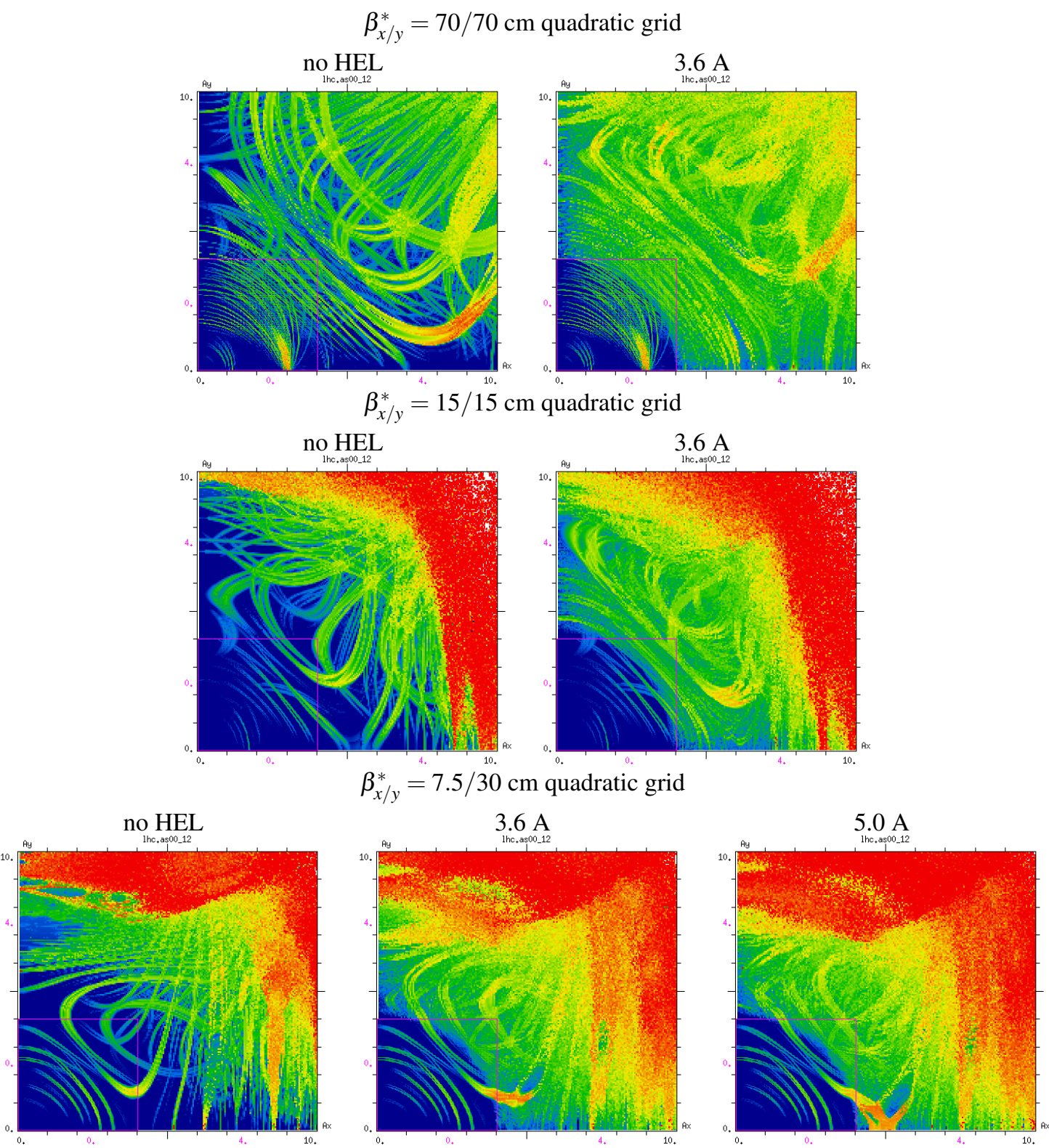

Figure 37. $\beta^{*}$-leveling, $Q^{\prime}=3, I_{\mathrm{MO}}=-550 \mathrm{~A}$, HEL installed at $-40 \mathrm{~m}$ : FMA analysis in amplitude space for offmomentum particles $\left(\frac{\Delta p}{p_{0}}=0.1 \sigma_{p}\right)$ up to $10 \sigma$ amplitude for a quadratric grid with and without HEL. 

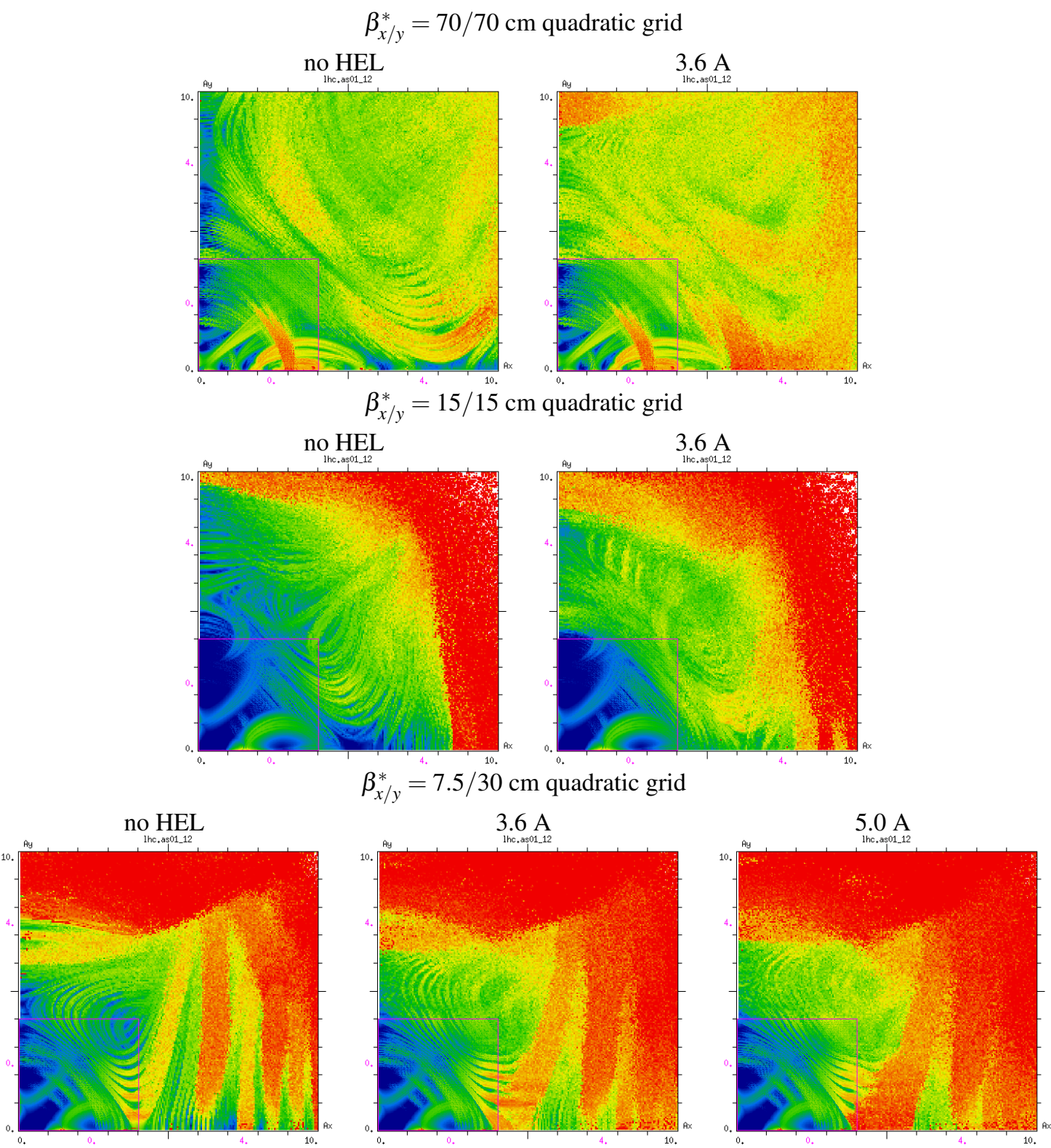

Figure 38. $\beta^{*}$-leveling, $Q^{\prime}=3, I_{\mathrm{MO}}=-550 \mathrm{~A}$, HEL installed at $-40 \mathrm{~m}$ : FMA analysis in amplitude space for onmomentum particles $\left(\frac{\Delta p}{p_{0}}=0\right)$ up to $10 \sigma$ amplitude for a quadratric grid with and without HEL. 
Appendix D: Losses versus amplitude

Appendix E: Losses versus amplitude at flat top

a. Losses vs amplitude: flat top and different chromaticity and octupole settings for HEL installed at $-40 \mathrm{~m}$
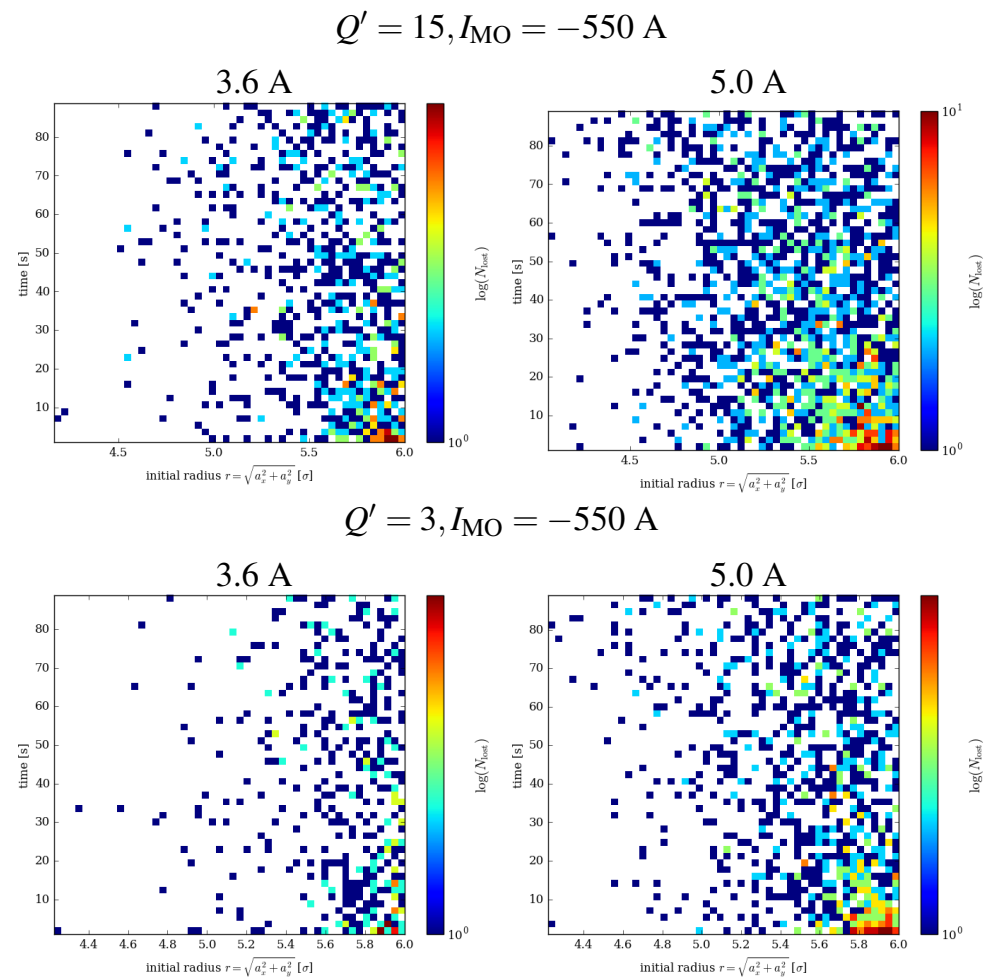

$$
Q^{\prime}=3, I_{\mathrm{MO}}=0 \mathrm{~A}
$$
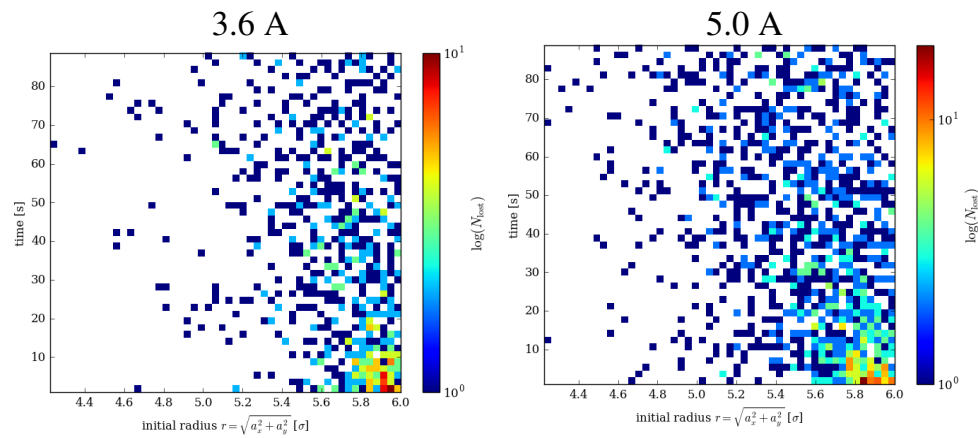

Figure 39. flat top, HEL installed at -40 m: Losses vs normalized radial amplitude $a_{r}=\sqrt{\left(a_{x}^{2}+a_{y}^{2}\right)}$, where $a_{x}$ and $a_{y}$ are normalized amplitudes in $\sigma$ and time for a uniform halo distribution between $4-6 \sigma$ and Gaussian in $\frac{\Delta p}{p_{0}}$ cut at $6 \sigma$. The case without HEL is not shown as only minimal losses are observed. 

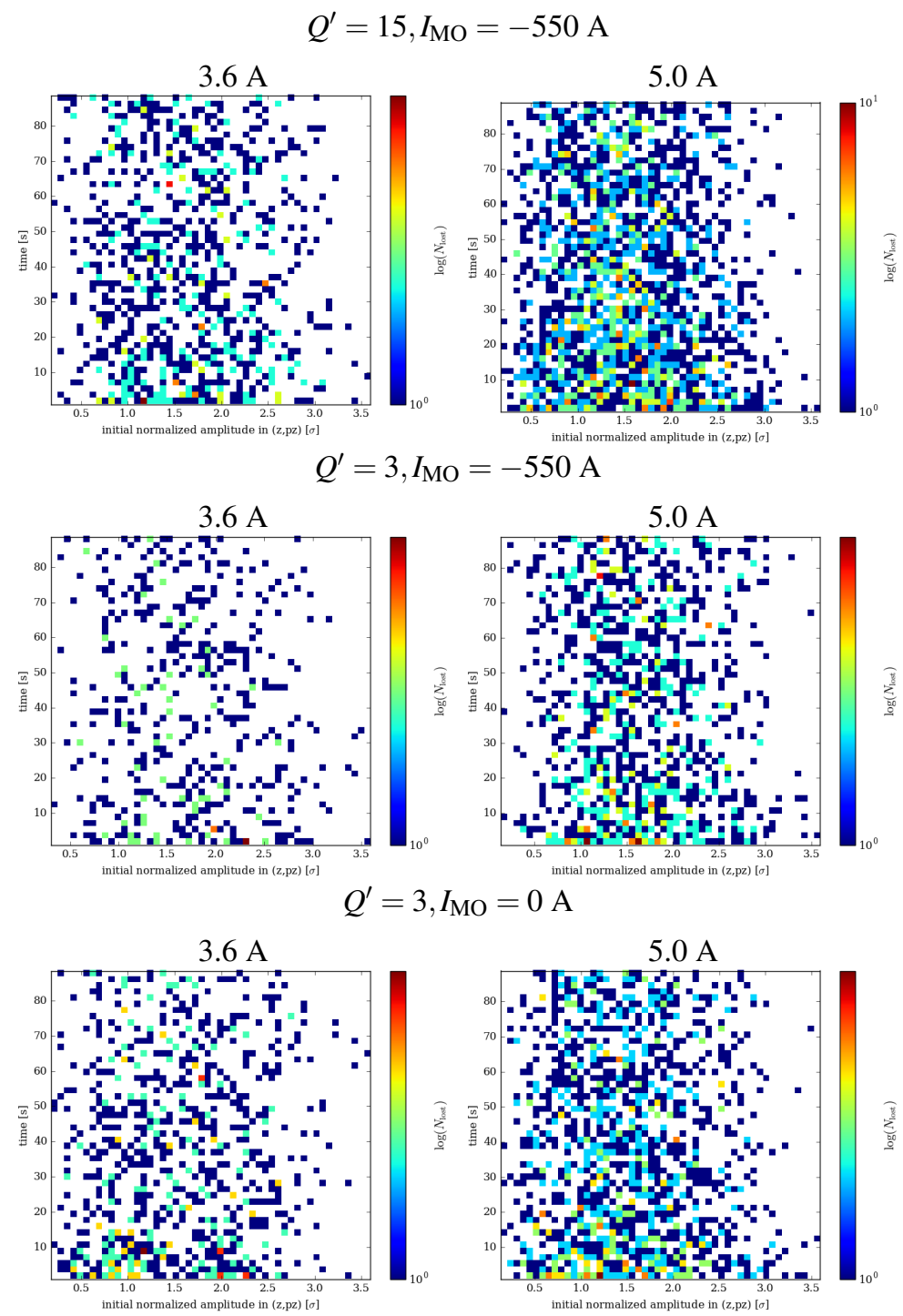

Figure 40. flat top, HEL installed at $-40 \mathrm{~m}$ : Losses vs normalized normalized longitudinal amplitude $a_{z}=$ $\sqrt{\left(a_{z}^{2}+a_{p z}^{2}\right)}$, where $a_{z}$ and $a_{p z}$ are normalized longitudinal coordinates in $\sigma$, and time for a uniform halo distribution between $4-6 \sigma$ and Gaussian in $\frac{\Delta p}{p_{0}}$ cut at $6 \sigma$. The case without HEL is not shown as only minimal losses are observed. 
b. Losses vs amplitude: flat top with magnet errors and HEL at $-40 \mathrm{~m}$ and $-88.6 \mathrm{~m}: Q^{\prime}=15, I_{\mathrm{MO}}=-550 \mathrm{~A}$

no errors
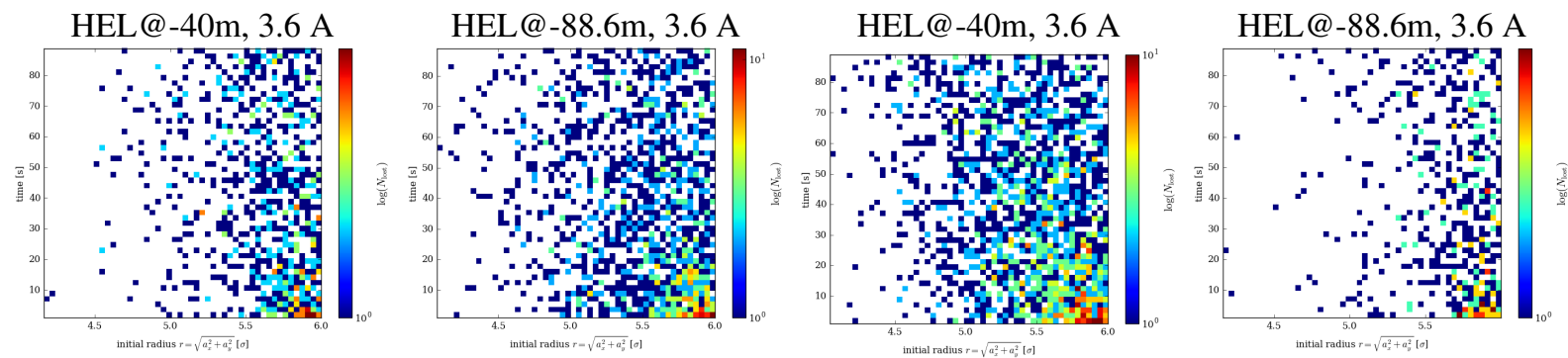

with errors
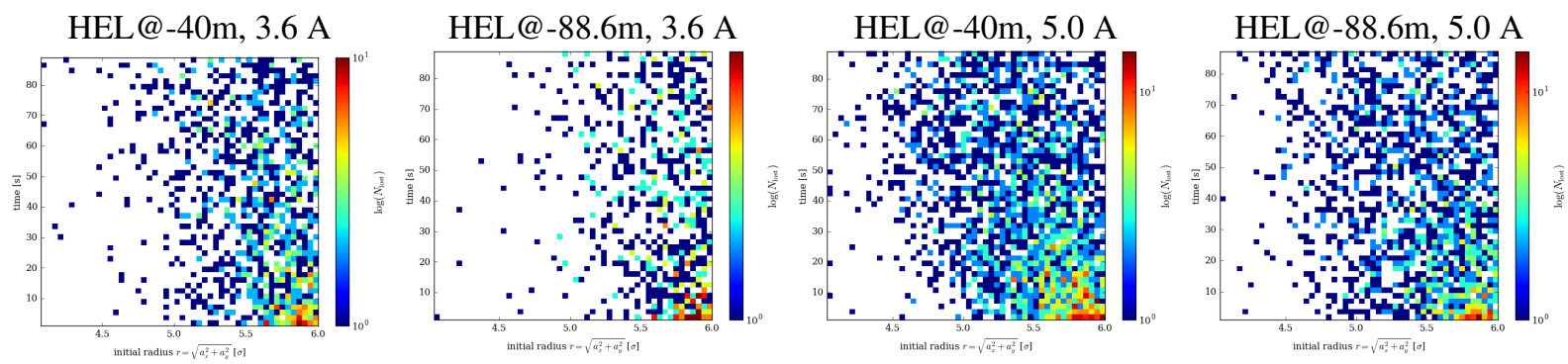

Figure 41. flat top with (bottom) and without (top) magnetic errors, $Q^{\prime}=15, I_{\mathrm{MO}}=-550 \mathrm{~A}$, HEL installed at $-40 \mathrm{~m}$ and -88.6 m: Losses vs normalized radial amplitude $a_{r}=\sqrt{\left(a_{x}^{2}+a_{y}^{2}\right)}$, where $a_{x}$ and $a_{y}$ are normalized amplitudes in $\sigma$ and time for a uniform halo distribution between $4-6 \sigma$ and Gaussian in $\frac{\Delta p}{p_{0}}$ cut at $6 \sigma$. The case without HEL is not shown as only minimal losses are observed. 
no errors

HEL@-40m,3.6 A

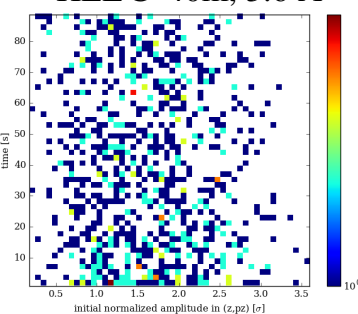

HEL@-40m, 3.6 A

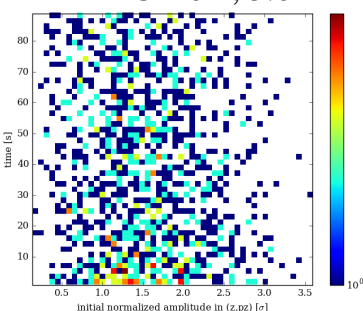

HEL@-88.6m, 3.6 A

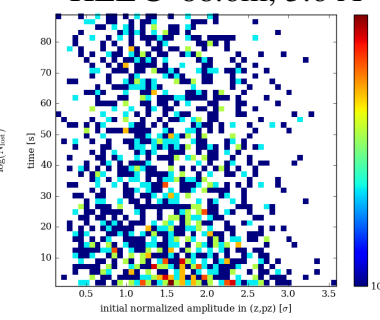

HEL@-40m, 3.6 A

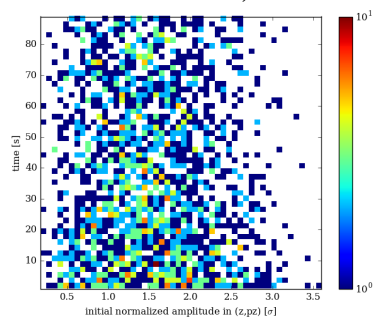

with errors

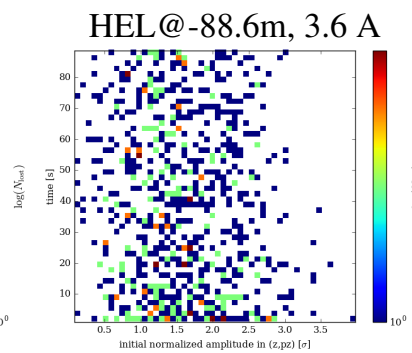

HEL@-40m, 5.0 A

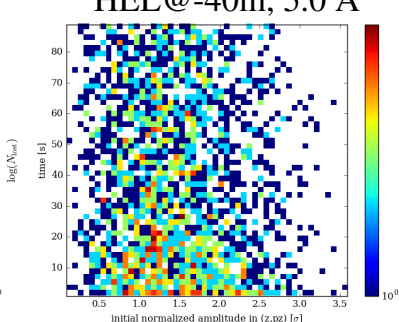

HEL@-88.6m, 5.0 A

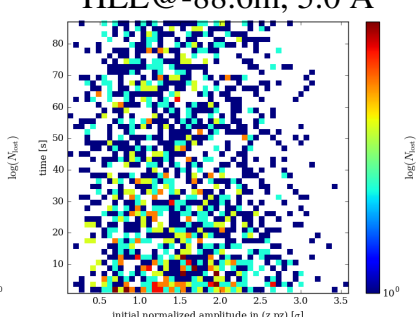

Figure 42. flat top with (bottom) and without (top) magnetic errors, $Q^{\prime}=15, I_{\mathrm{MO}}=-550 \mathrm{~A}$, HEL installed at $40 \mathrm{~m}$ and -88.6 m: Losses vs normalized normalized longitudinal amplitude $a_{z}=\sqrt{\left(a_{z}^{2}+a_{p z}^{2}\right)}$, where $a_{z}$ and $a_{p z}$ are normalized longitudinal coordinates in $\sigma$, and time for a uniform halo distribution between $4-6 \sigma$ and Gaussian in $\frac{\Delta p}{p_{0}}$ cut at $6 \sigma$. The case without HEL is not shown as only minimal losses are observed. 
c. Losses vs amplitude: flat top with pulsing and $Q^{\prime}=15, I_{\mathrm{MO}}=-550 \mathrm{~A}$

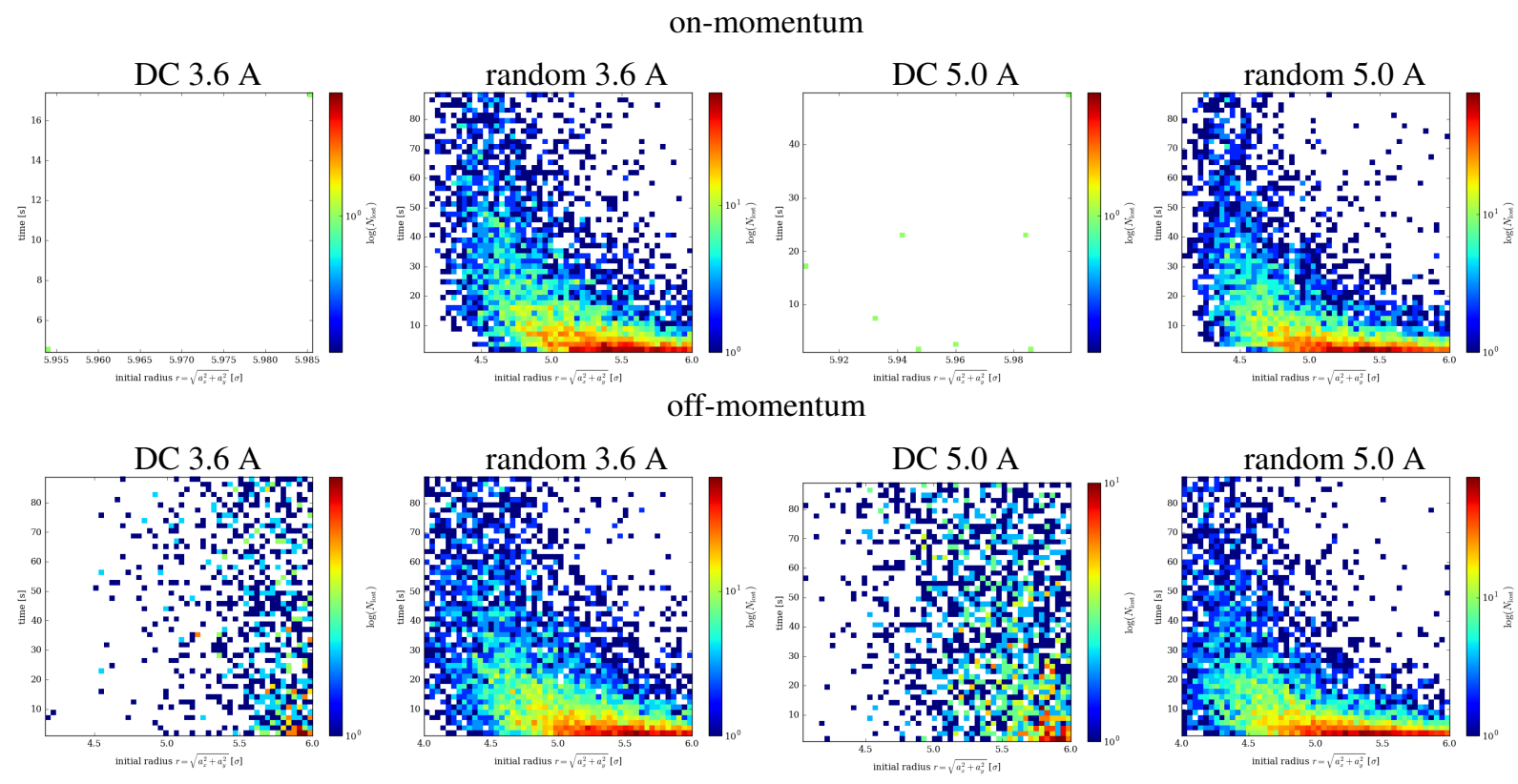

Figure 43. flat top, $Q^{\prime}=15, I_{\mathrm{MO}}=-550 \mathrm{~A}$, HEL installed at $-40 \mathrm{~m}$, random pulsing: Losses vs normalized radial amplitude $a_{r}=\sqrt{\left(a_{x}^{2}+a_{y}^{2}\right)}$, where $a_{x}$ and $a_{y}$ are normalized amplitudes in $\sigma$ and time for a uniform halo distribution between 4- $6 \sigma$ and on-momentum $\frac{\Delta p}{p_{0}}=0$ (top) and Gaussian distribution in $\frac{\Delta p}{p_{0}}$ cut at $6 \sigma$ (bottom). The case without HEL is not shown as only minimal losses are observed. The losses for DC current and on-momentum are also so small that they hardly appear in the plots.

off-momentum
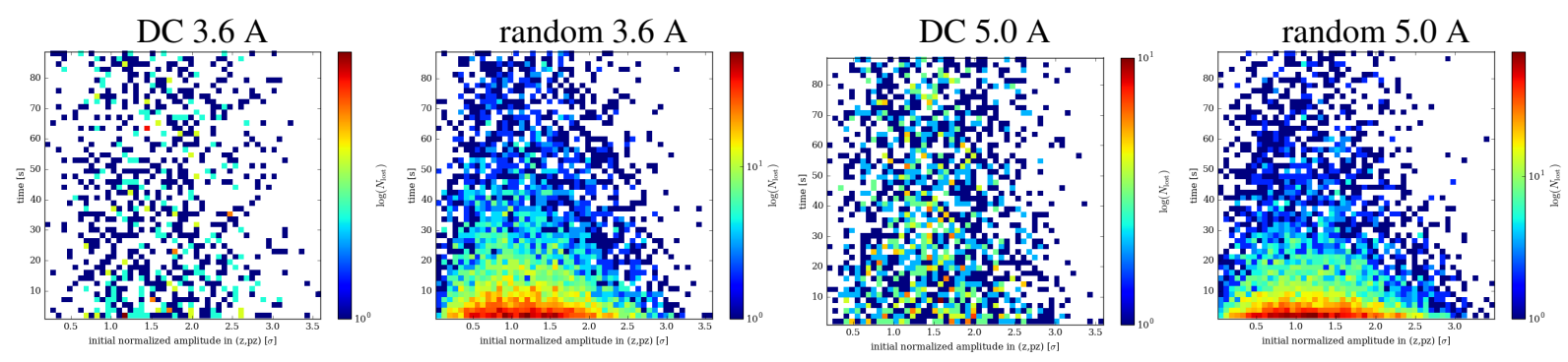

Figure 44. flat top, $Q^{\prime}=15, I_{\mathrm{MO}}=-550 \mathrm{~A}$, HEL installed at $-40 \mathrm{~m}$, random pulsing: Losses vs normalized normalized longitudinal amplitude $a_{z}=\sqrt{\left(a_{z}^{2}+a_{p z}^{2}\right)}$, where $a_{z}$ and $a_{p z}$ are normalized longitudinal coordinates in $\sigma$, and time for a uniform halo distribution between $4-6 \sigma$ and Gaussian distribution in $\frac{\Delta p}{p_{0}}$ cut at $6 \sigma$. The case without HEL is not shown as only minimal losses are observed.

1. Losses vs amplitude: : $\beta^{*}$-leveling cases for HEL installed at $-\mathbf{4 0} \mathrm{m}$ 


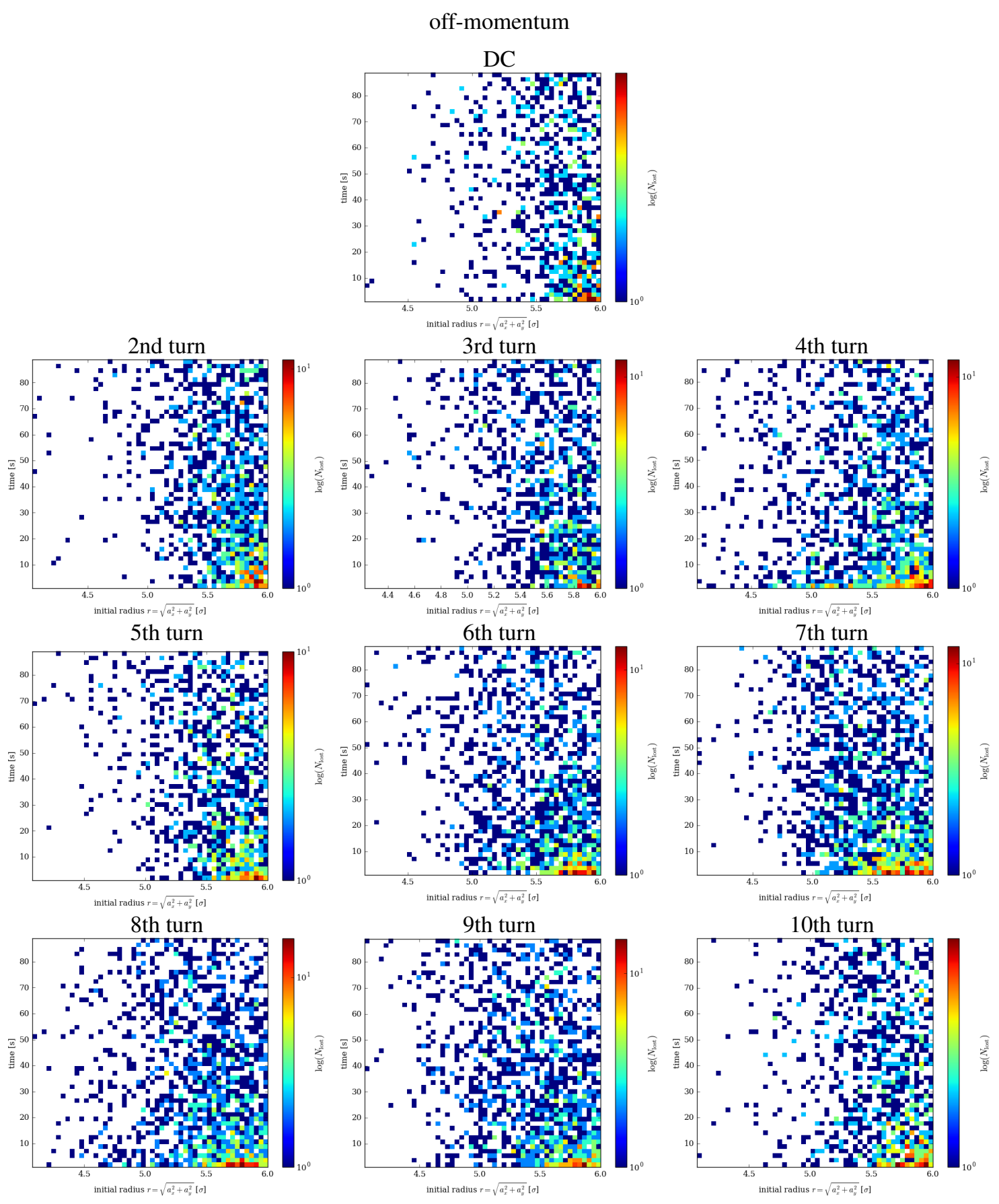

Figure 45. flat top, $Q^{\prime}=15, I_{\mathrm{MO}}=-550 \mathrm{~A}$, HEL installed at $-40 \mathrm{~m}$, resonant pulsing: Losses vs normalized radial amplitude $a_{r}=\sqrt{\left(a_{x}^{2}+a_{y}^{2}\right)}$, where $a_{x}$ and $a_{y}$ are normalized amplitudes in $\sigma$ and time for a uniform halo distribution between $4-6 \sigma$ and Gaussian in $\frac{\Delta p}{p_{0}}$ cut at $6 \sigma$. The case without HEL and for on-momentum are not shown as only minimal losses are observed. 


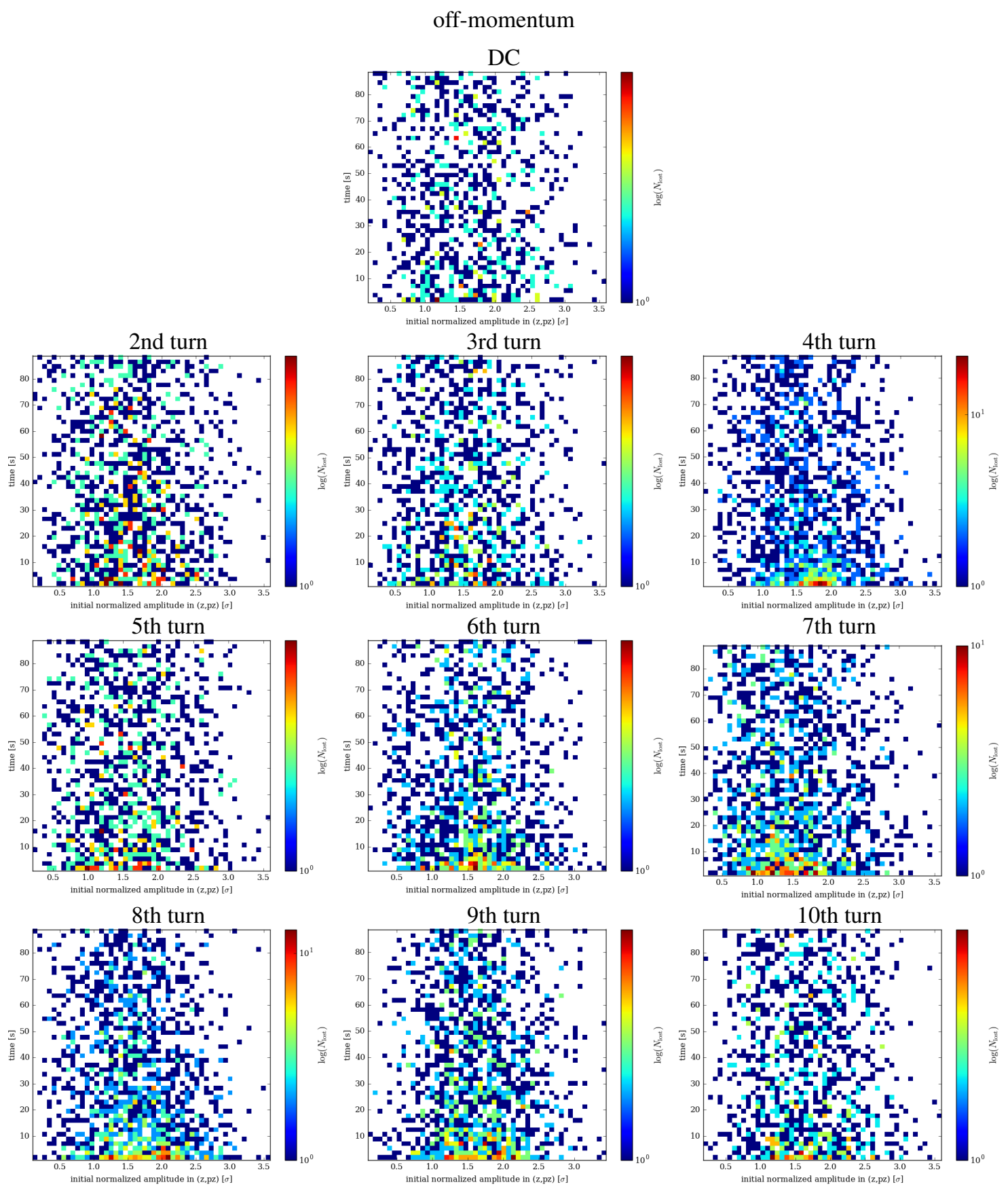

Figure 46. flat top, $Q^{\prime}=15, I_{\mathrm{MO}}=-550 \mathrm{~A}$, HEL installed at $-40 \mathrm{~m}$, resonant pulsing: Losses vs normalized normalized longitudinal amplitude $a_{z}=\sqrt{\left(a_{z}^{2}+a_{p z}^{2}\right)}$, where $a_{z}$ and $a_{p z}$ are normalized longitudinal coordinates in $\sigma$, and time for a uniform halo distribution between $4-6 \sigma$ and Gaussian in $\frac{\Delta p}{p_{0}}$ cut at $6 \sigma$. The case without HEL is not shown as only minimal losses are observed. 

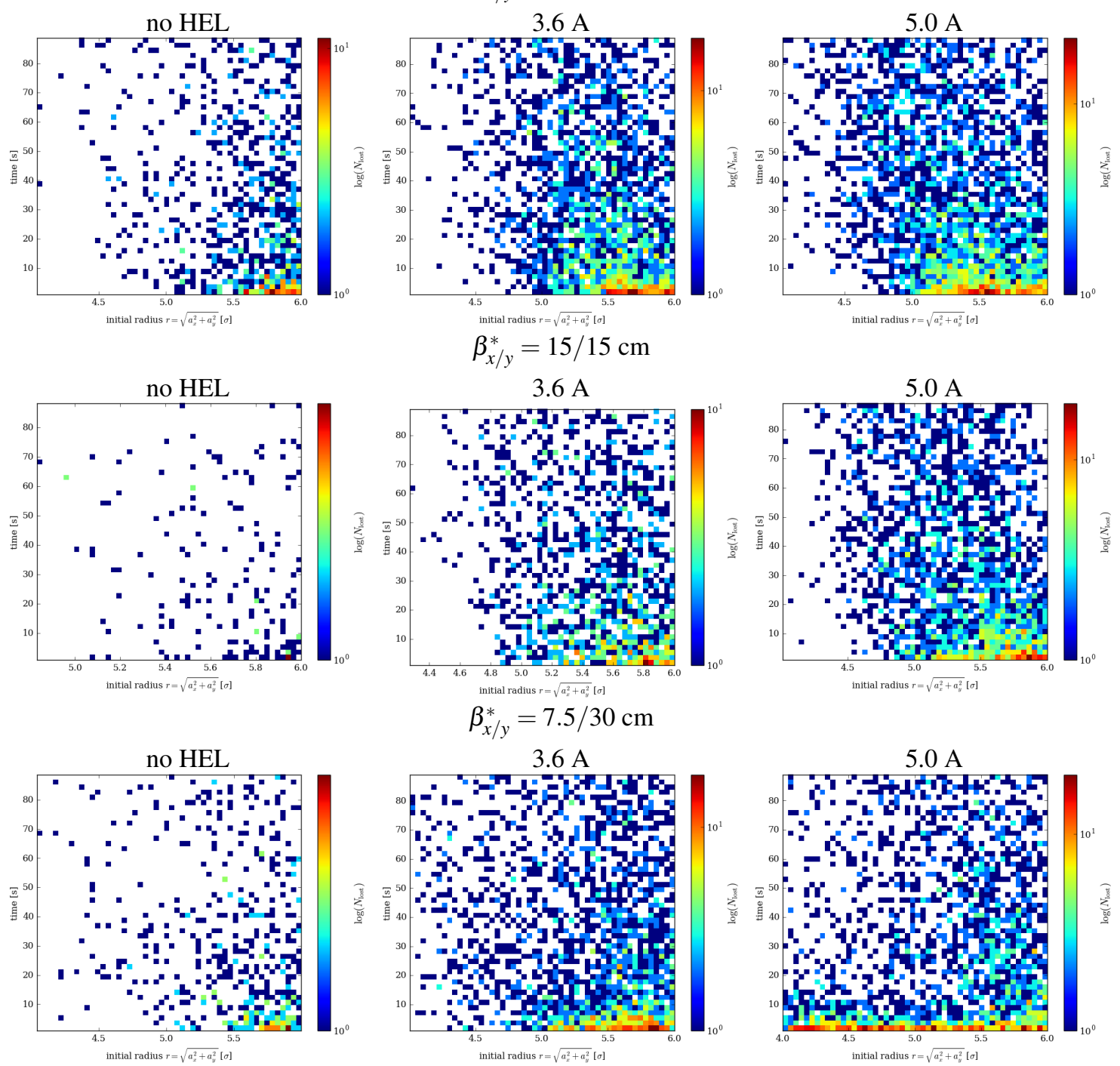

Figure 47. $\beta^{*}$-leveling, $Q^{\prime}=3, I_{\mathrm{MO}}=-550 \mathrm{~A}$, HEL installed at $-40 \mathrm{~m}$ : Losses vs normalized radial amplitude $a_{r}=\sqrt{\left(a_{x}^{2}+a_{y}^{2}\right)}$, where $a_{x}$ and $a_{y}$ are normalized amplitudes in $\sigma$ and time for a uniform halo distribution between $4-6 \sigma$ and Gaussian distribution in $\frac{\Delta p}{p_{0}}$ cut at $6 \sigma$. The case without HEL and for on-momentum $\left(\frac{\Delta p}{p_{0}}\right)$ are not shown as only minimal losses are observed. 

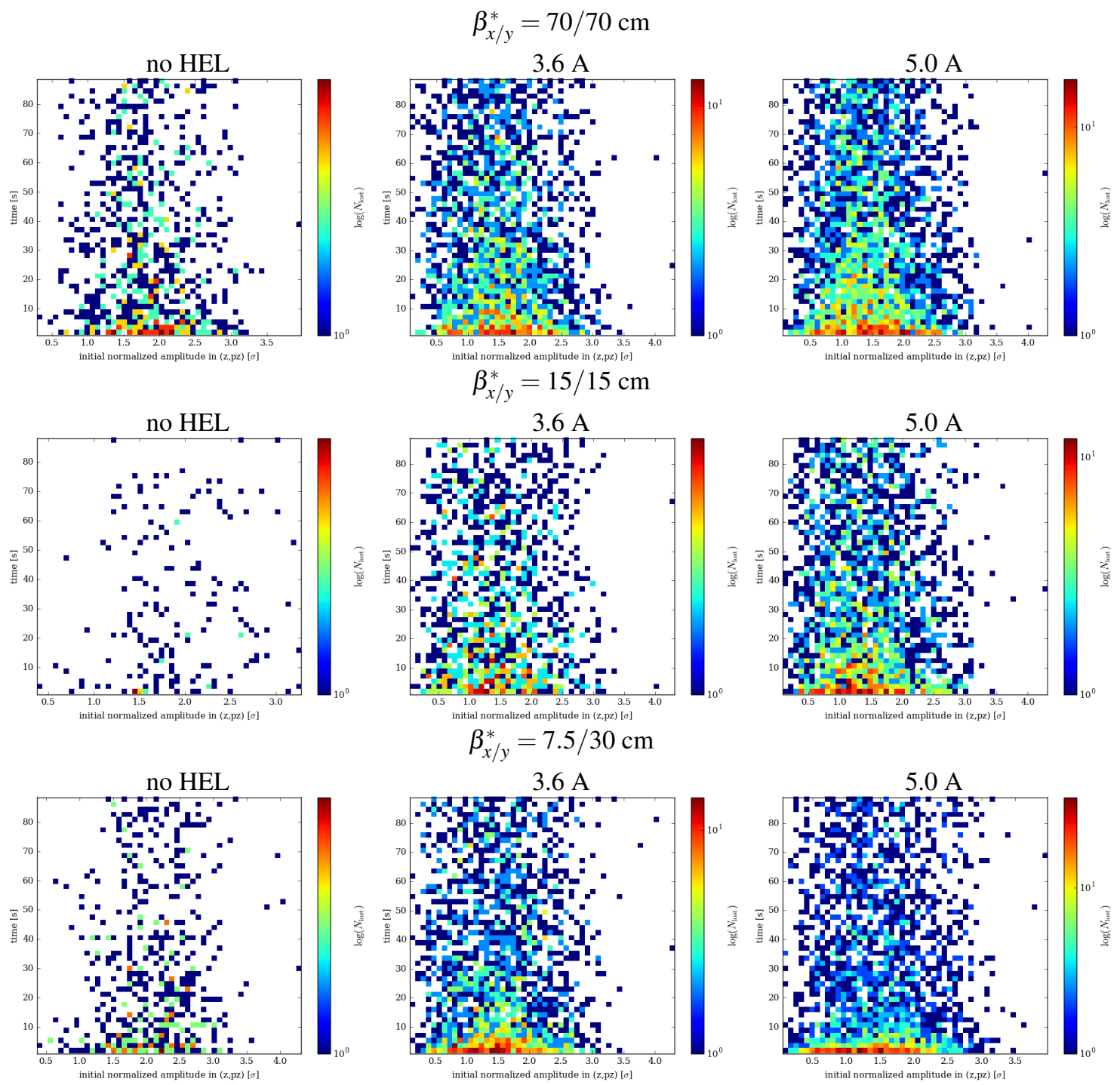

Figure 48. $\beta^{*}$-leveling, $Q^{\prime}=3, I_{\mathrm{MO}}=-550 \mathrm{~A}$, HEL installed at $-40 \mathrm{~m}$ : Losses vs normalized normalized longitudinal amplitude $a_{z}=\sqrt{\left(a_{z}^{2}+a_{p z}^{2}\right)}$, where $a_{z}$ and $a_{p z}$ are normalized longitudinal coordinates in $\sigma$, and time for a uniform halo distribution between $4-6 \sigma$ and Gaussian in $\frac{\Delta p}{p_{0}}$ cut at $6 \sigma$. 
[1] G. Stancari, A. Valishev, G. Annala, G. Kuznetsov, V. Shiltsev, D. A. Still, and L. G. Vorobiev. Collimation with hollow electron beams. Phys. Rev. Lett., 107:084802, Aug 2011.

[2] Giulio Stancari, Valentina Previtali, Alexander Valishev, Roderik Bruce, Stefano Redaelli, Adriana Rossi, and Belen Salvachua Ferrando. Conceptual design of hollow electron lenses for beam halo control in the Large Hadron Collider. 2014.

[3] V. Previtali, G. Stancari, A. Valishev, and S. Redaelli. Numerical simulations of a proposed hollow electron beam collimator for the LHC upgrade at CERN. 2013.

[4] A. Valishev. Simulation Study of Hollow Electron Beam Collimation for LHC. 2014.

[5] Xiao-Long Zhang, Kip Bishofberger, Vsevolod Kamerdzhiev, Valery Lebedev, Vladimir Shiltsev, Randy Thurman-Keup, and Alvin Tollestrup. Generation and diagnostics of uncaptured beam in the fermilab tevatron and its control by electron lenses. Phys. Rev. ST Accel. Beams, 11:051002, May 2008.

[6] Miriam Fitterer, Giulio Stancari, and Valishev. Effect of pulsed hollow e-lens operation on the beam core. 2016.

[7] Elias Metral, Gianluigi Arduini, Danilo Banfi, Javier Barranco Garcia, Nicolo Biancacci, Oliver BrÃijning, Riccardo De Maria, Massimo Giovannozzi, Wolfgang HÃúfle, Kevin Li, Juan Esteban Muller, Yannis Papaphilippou, Tatiana Pieloni, Benoit Salvant, Elena Shaposhnikova, Claudia Tambasco, Alexander Valishev, Daniel Valuch, and Andrzej Wolski. HL-LHC Operational Scenarios. High Luminosity LHC. May 2015.

[8] private communication E. Mètral.

[9] A Valishev, Y Alexahin, V Lebedev, and D Shatilov. Simulation of beam-beam effects and tevatron experience. Journal of Instrumentation, 7(12):P12002, 2012.

[10] Low frequency noise and resonant excitation md. 